\title{
Mediastinum
}

\author{
B. KRUg, O. Schulte
}

17.1 Anatomische Grundlagen 665

17.1.1 Topographische Anatomie 665

17.1.2 Projektionsradiographie 666

17.1.3 Schnittbilddiagnostik 667

17.2 Fehlbildungen 673

17.3 Entzündungen 678

17.3.1 Akute Mediastinitis 678

17.3.2 Chronische Mediastinitis 679

17.4 Mediastinale Raumforderungen 680

17.4.1 Allgemeines 680

17.4.2 Primäre Mediastinaltumoren 683

17.4.3 Sekundäre Mediastinaltumoren 699

17.4.4 Mediastinale Lymphadenopathien 707

17.4.5 Pseudotumoren 718

17.5 Sonstige Erkrankungen 723

17.5.1 Mediastinalemphysem 723

17.5.2 Mediastinalverlagerungen 724

Literatur 727

D as Mediastinum bezeichnet den mittleren Anteil der Brusthöhle und wird in das vordere, mittlere und hintere Mediastinum unterteilt. Zur Diagnostik dienen Thoraxübersichtsaufnahmen und Schnittbildverfahren wie CT und MRT, die auf Grund der überlagerungsfreien Abbildung und des größeren Weichteilkontrastes der Projektionsradiographie überlegen sind. Die transösophageale Endosonographie macht eine Beurteilung der Ösophaguswand und des periösophagealen Mediastinums möglich. Angeborene Fehlbildungen des Bronchialbaums und des neuroenteralen Kanals sind überwiegend zystische Mediastinaltumoren (z.B. bronchiogene $\mathrm{Zy}$ sten). Bei entzündlichen Erkrankungen sind akute und chronische Mediastinitis zu unterscheiden. Als mediastinale Raumforderungen kommen insbesondere Thymom, Thymuskarzinom, teratogene Tumoren, Lymphadenopathien, maligne Lymphome und Lymphknotenmetastasen in Betracht.
17.1

\section{Anatomische Grundlagen}

\subsection{1}

\section{Topographische Anatomie}

Das Mediastinum (ableitbar aus lat. in medio: in der Mitte, stare: stehen; auch „medium intestinum“) bezeichnet den mittleren Anteil der Brusthöhle. Es wird ventral vom Sternum, dorsal von der Wirbelsäule, seitlich von der mediastinalen Pleura und kaudal vom Zwerchfell begrenzt. Nach kranial geht das Mediastinum kontinuierlich in die supraklavikulären, zervikalen und prävertebralen Weichteile über. Nach kaudal bestehen über den Hiatus oesophagei und den Hiatus aorticus Verbindungen zum Retroperitoneum. Das Mediastinum beinhaltet - eingelagert in Fett- und Bindegewebe - das Herz, den Ösophagus, die Trachea, den Thymus und große Gefäße und Nerven.

Das Mediastinum wird bei einer sagittalen anatomisch-topographischen Gliederung in das vordere, das mittlere und das hintere Mediastinum (Holzknecht- oder Prä- bzw. Paravertebralraum) unterteilt. Die Zuordnung der einzelnen Organstrukturen zu den drei Kompartimenten erfolgt nach anatomischen, pathogenetischen oder diagnostischen Gesichtspunkten, sodass sich bei dem Vergleich einzelner Autoren Variationen ergeben. So wird das Herz gelegentlich dem vorderen, meist jedoch dem mittleren Mediastinum zugerechnet. Das vordere Mediastinum wird durch die Rückfläche des Sternums und die ventralen Konturen des Perikards, der Trachea und der oberen Hohlvene begrenzt. Das mittlere Mediastinum liegt zwischen vorderer Trachealwand bzw. ventralem Perikard und den Vorderkanten der Brustwirbelkörper. Die Grenzen des hinteren Mediastinums sind ventral durch die Wirbelkörpervorderkanten und dorsal durch die hintere Thoraxwand definiert. Zusätzlich wird das Mediastinum in eine obere und eine untere Hälfte unterteilt, die durch eine horizontale vom Sternalwinkel über die obere perikardiale Umschlagfalte bis zur Grundplatte des 4. Brustwirbelkörpers (BWK) reichenden Ebene getrennt sind. 

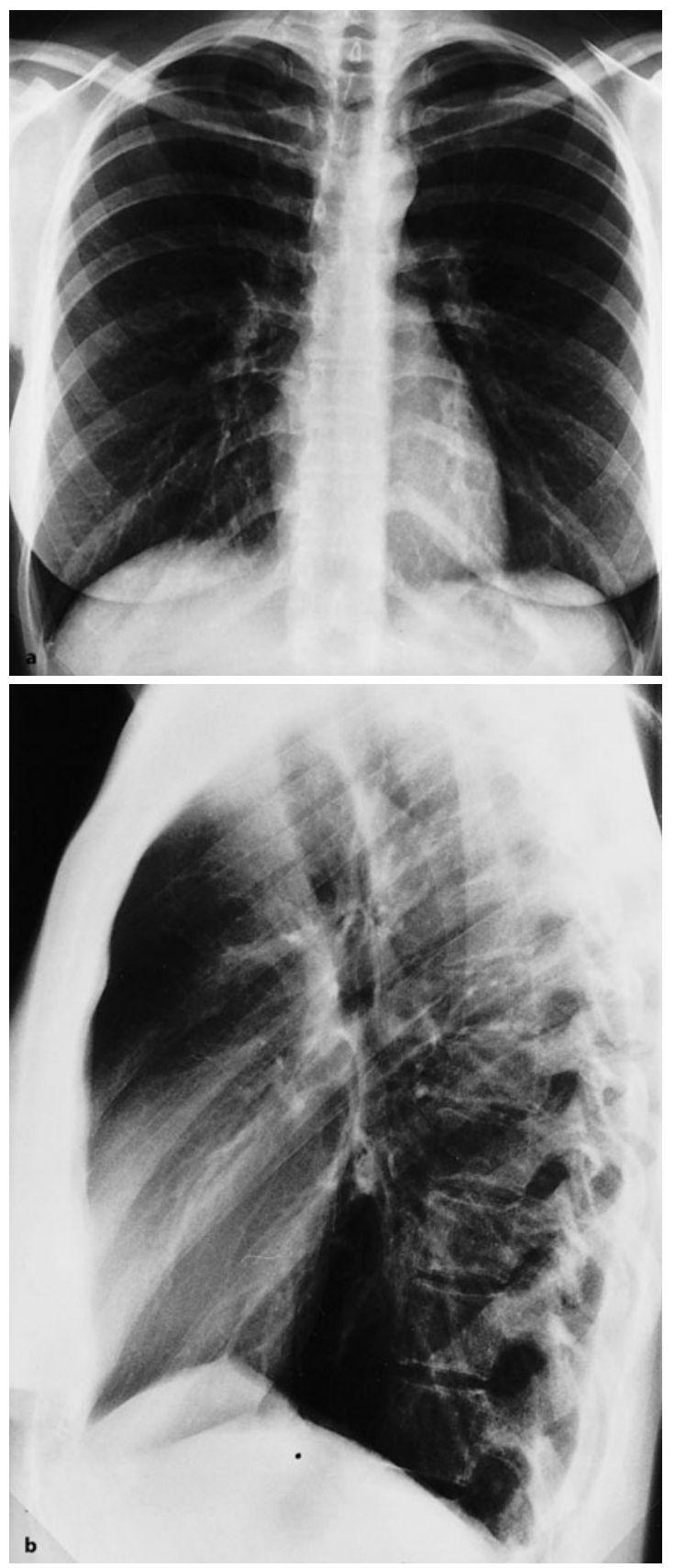

Abb. 17.1 a, b. Projektionsradiographischer Normalbefund de Mediastinums. a Thoraxübersichtsaufnahme im sagittalen und $\mathbf{b}$ im seitlichen Strahlengang
Das vordere Mediastinum enthält den Thymus, Lymphknoten und den N. phrenicus. Das mittlere Mediastinum beinhaltet das Herz, die Aorta ascendens, die Vv. cava superior und inferior, den Ösophagus, die Trachea einschließlich der Stammbronchien, Lymphknoten und den N. vagus. Im hinteren Mediastinum liegen der Aortenbogen und die Aorta descendens, die Vv. azygos und hemiazygos, Lymphknoten, der Truncus sympathicus und Interkostalarterien.

\subsection{2 \\ Projektionsradiographie}

Mit Ausnahme von Luft in Hohlorganen (zentrale Atemwege, Ösophagus) und von Verkalkungen (Aorten- und Trachealsklerose, Lymphknotenverkalkungen) haben alle physiologischen und pathologischen Strukturen des Mediastinums etwa die gleiche Dichte, sodass sie auf Thoraxübersichtsaufnahmen nicht voneinander zu differenzieren sind (Abb. 17.1 a).

Aorta und Truncus pulmonalis sind an ihren randständigen Außenkonturen, die Aorta gelegentlich auch an pathognomonischen Wandverkalkungen erkennbar. Der Azygoslymphknoten grenzt sich im Sagittalbild als laterale Ausbuchtung des rechten oberen Mediastinums kranial der Aorta ascendens und im Seitbild als rundlich-ovaläre Verdichtung ventrokranial der Stammbronchien ab. Aufgrund der Anhebung durch den linken Vorhof verläuft der linke Stammbronchus stärker horizontal als der rechte, im Seitbild liegt also der luftgefüllte Queranschnitt des linken Stammbronchus höher als der des rechten (Abb. 17.1b). Das Ösophaguslumen kann durch röntgennegatives (Luft) oder röntgenpositives Kontrastmittel erkennbar sein. Durch die unmittelbare topographische Beziehung der Mediastinalorgane zur Lunge entstehen pleuromediastinale Berührungsflächen. Treffen Röntgenstrahlen orthograd auf diese Grenzflächen, so bilden sich im Röntgensummationsbild scharf begrenzte, sog. Pleuralinien ab (Beyer 1980; Lange 1996; Neufang u. Beyer 1980; Neufang u. Bulo 1981). Die digitale Projektionsradiographie ermöglicht durch ihren im Vergleich zur analogen Technik größeren Expositionsspielraum eine zuverlässigere Abgrenzung der Pleuralinien. Folgende Formen werden unterschieden (Abb. 17.2).

\footnotetext{
Pleuralinien

- Subclavia-Herz-Bogen

$\checkmark$ Die vorderen Umschlagfalten der Pleura verlaufen beidseits der V. subclavia folgend von kraniolateral nach kaudoventral, sodass sie in Höhe des Aortenbogens und des Herzens einander anliegen. Hieraus resultiert eine Y-förmige Verdichtung, deren Basis am Herz beginnt und
} 


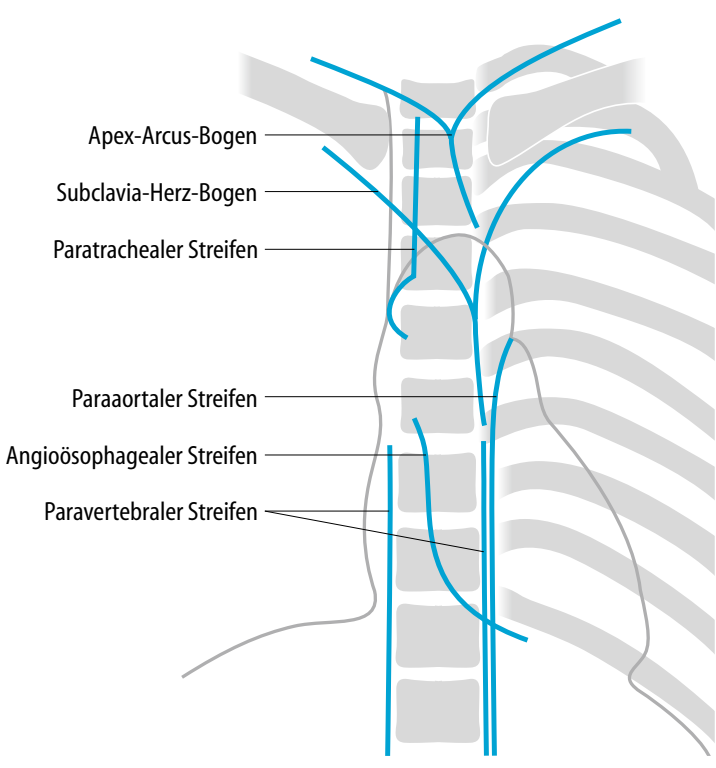

Abb. 17.2. Schematische Wiedergabe der Pleuralinien. (Mod. nach Lange 1996)

deren Schenkel bis zu den Unterkanten der Sternoklavikulargelenke reichen.

- Apex-Arcus-Bogen

$\checkmark$ Die hinteren Umschlagfalten der Pleura mediastinalis ziehen in Begleitung der 2. Rippe nach medial, treffen in Höhe des Zwischenwirbelraumes BWK $3 / 4$ auf die Pleura medastinalis der Gegenseite und ziehen als vertikaler Streifen zum Aortenbogen.

- Azygoösophagealer Streifen

$\checkmark$ Auf der rechten Seite grenzt die Lunge prävertebral an die V. azygos und den Ösophagus. Die Pleuraumschlagsfalte bildet eine fast in der Medianebene nach unten verlaufenden streifenförmige Verdichtung.

- Paravertebraler/paraspinaler Streifen

$\nabla$ Die dorsalen Lungenabschnitte liegen der paravertebralen Muskulatur seitlich an. Hierdurch entsteht eine vertikal verlaufende paravertebrale Verdichtung, die auf der linken Seite praktisch immer erkennbar ist und auf der rechten Seite dann von der Verdichtung der Wirbelsäule differenziert werden kann, wenn der Paravertebralraum durch Osteophyten, Hämatome oder Tumore verbreitert ist.

- Paraaortaler Streifen

$\nabla$ Er entspricht der lateralen Kontur der Aorta descendens und stellt im Gegensatz zum paravertebralen Streifen eine strichförmige Transparenzerhöhung dar.

- Para- und retrotrachealer Streifen

$\checkmark$ Die rechte Lunge liegt medial der laterodorsalen Trachealwand an. Diese Nachbarschaftsbeziehung findet ihr röntgenmorphologisches Korrelat in einer paratrachealen (Sagittalbild) und retrotrachealen (Seitbild) streifenförmigen Verdichtung, die im Normalfall nicht breiter als $3-4 \mathrm{~mm}$ ist.

\subsection{3}

\section{Schnittbilddiagnostik}

Aufgrund der überlagerungsfreien Abbildung und des größeren Weichteilkontrastes ermöglichen die Schnittbildverfahren Computertomographie (CT) und Magnetresonanztomographie (MRT) eine der Projektionsradiographie überlegene Darstellung der Topographie (Abb.17.3, 17.4; Chukwuemeka 1997; Lange 1996; Leppert 1998; Schaefer-Prokop 1998; Wegener 1992).

Die großen arteriellen und venösen Gefäße gliedern das Mediastinum. Die auf Transversalschichten quer angeschnittene Aorta ascendens und Aorta descendens und ihre Abgangsgefäße sind ausgehend vom Aortenbogen leicht zu identifizieren. Die linke A. subclavia, die am weitesten dorsal aus dem Aortenbogen entspringt, wölbt sich gegen die linke Lungenspitze vor. Die in Kaliber und Verlauf stark variierende linke V. brachiocephalica (anonyma) liegt kranioventral des Aortenbogens. Die rechte V. brachiocephalica bildet die rechte obere Mediastinalkontur. Der Zusammenfluss beider Venen zur V. cava superior ist leicht zu identifizieren. Die V. cava superior verläuft rechts laterodorsal der Aorta ascendens bis zu ihrer Einmündung in den rechten Vorhof. Zur Abgrenzung von Querschnitten der V. subclavia und der Vv. jugulares internae und externae von Lymphknoten ist computertomographisch eine transvenöse Kontrastierung der Gefäßlumina hilfreich. V. subclavia und V.axillaris liegen ventral der entsprechenden Arterien und lassen sich dorsal des M. pectoralis minor bis in die Axillen verfolgen.

Conus und Truncus pulmonalis sind durch ihre Einbettung in das subepikardiale Fettgewebe sowohl computer- als auch MR-tomographisch gut beurteilbar. Die rechte A. pulmonalis hat intraperikardial einen Durchmesser von 12-15 mm und zieht dorsal der V. cava superior und ventral des Stammbronchus zum rechten Hilus. Die linke A. pulmonalis verläuft nur kurzstreckig intraperikardial, überkreuzt den linken Stammbronchus und zieht dorsal zum linken Hilus. Die Oberlappenvenen verlaufen ventrolateral der Arterien und Bronchien zum linken Vorhof. Die Einmündungen der Unterlappenvenen sind an den lateralen Rändern des linken Vorhofs erkennbar.

Die $V$. azygos verläuft rechts paravertebral im hinteren Mediastinum. Sie ist je nach Fettgehalt des umgebenden Gewebes ab einem Durchmesser von 3-5 mm erkennbar. In Höhe BWK 4-6 kreuzt sie in einem laterokonvexen Bogen zu der im mittleren Mediastinum gelegenen V. cava superior (Abb. 17.5). Kranial des Azygosbogens wölbt sich die Lunge dorsal der Trachea als supraazygealer Rezessus gegen das Mediastinum vor. Kaudal des Azygosbogens bildet sie in Höhe des Hilus und des Herzens den 

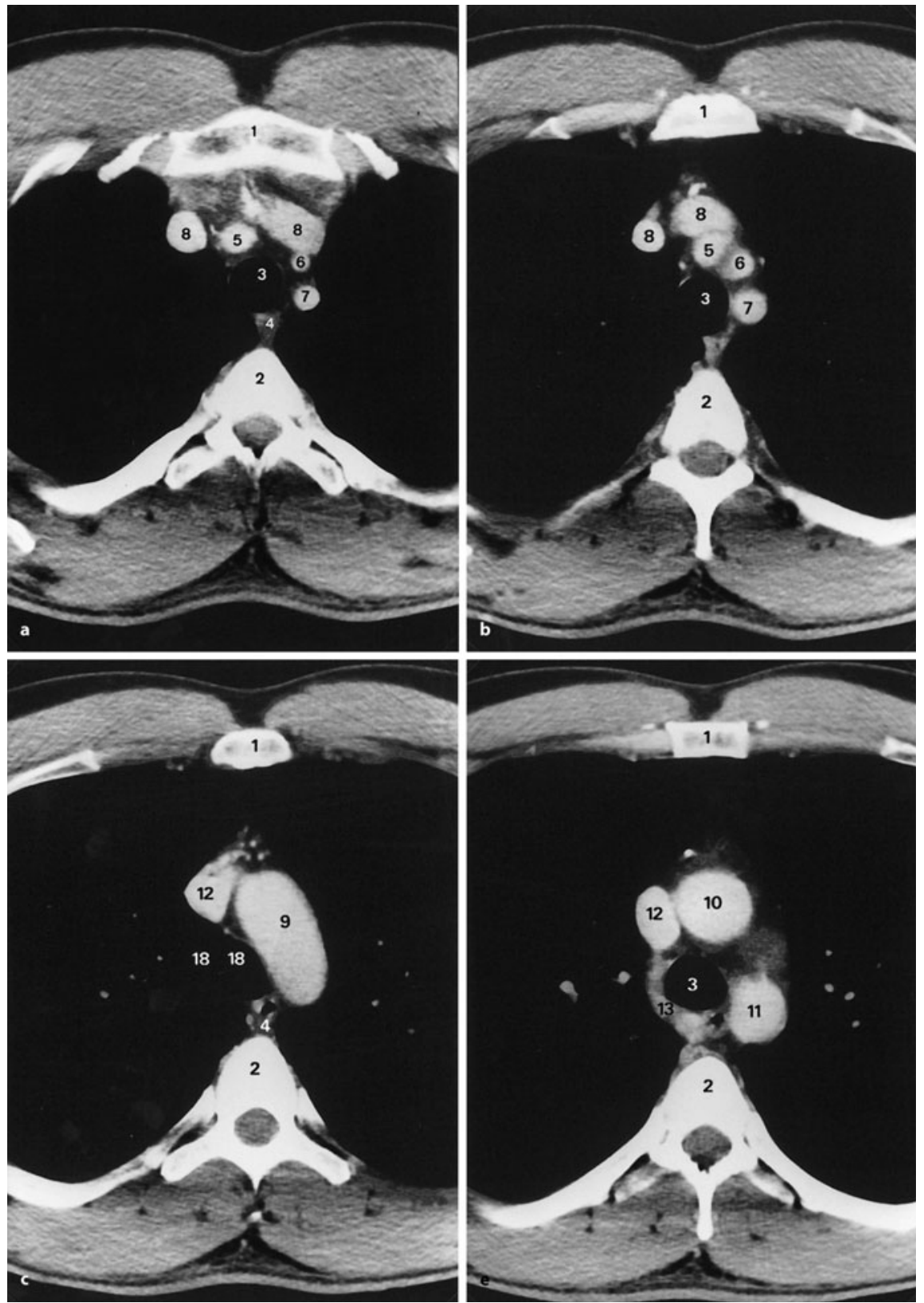

Abb. 17.3 a-d. Topographische Anatomie des Mediastinums in der Computertomographie. 1 Sternum, 2 Wirbelsäule, 3 Trachea, 4 Ösophagus, 5 A. brachiozephalica, 6 A. carotis, 7 A. subclavia, 8 V. subclavia, 9 Aortenbogen, 10 Aorta ascendens, 11 Aorta descendens, 12 V. cava superior, 13 V. azygos,

$14 \mathrm{~V}$. hemiazygos, 15 Truncus pulmonalis, 16 A. pulmonalis dextra bzw. sinistra, 17 Segmentaufzweigung der A. pulmonalis, 18 Stammbronchus, 19 rechter Vorhof, 20 linker Vorhof, 21 Lungenvene, 22 Bulbus aortae, 23 rechte Herzkammer, 24 linke Herzkammer, 25 Sinus coronarius, 26 V. cava inferior 

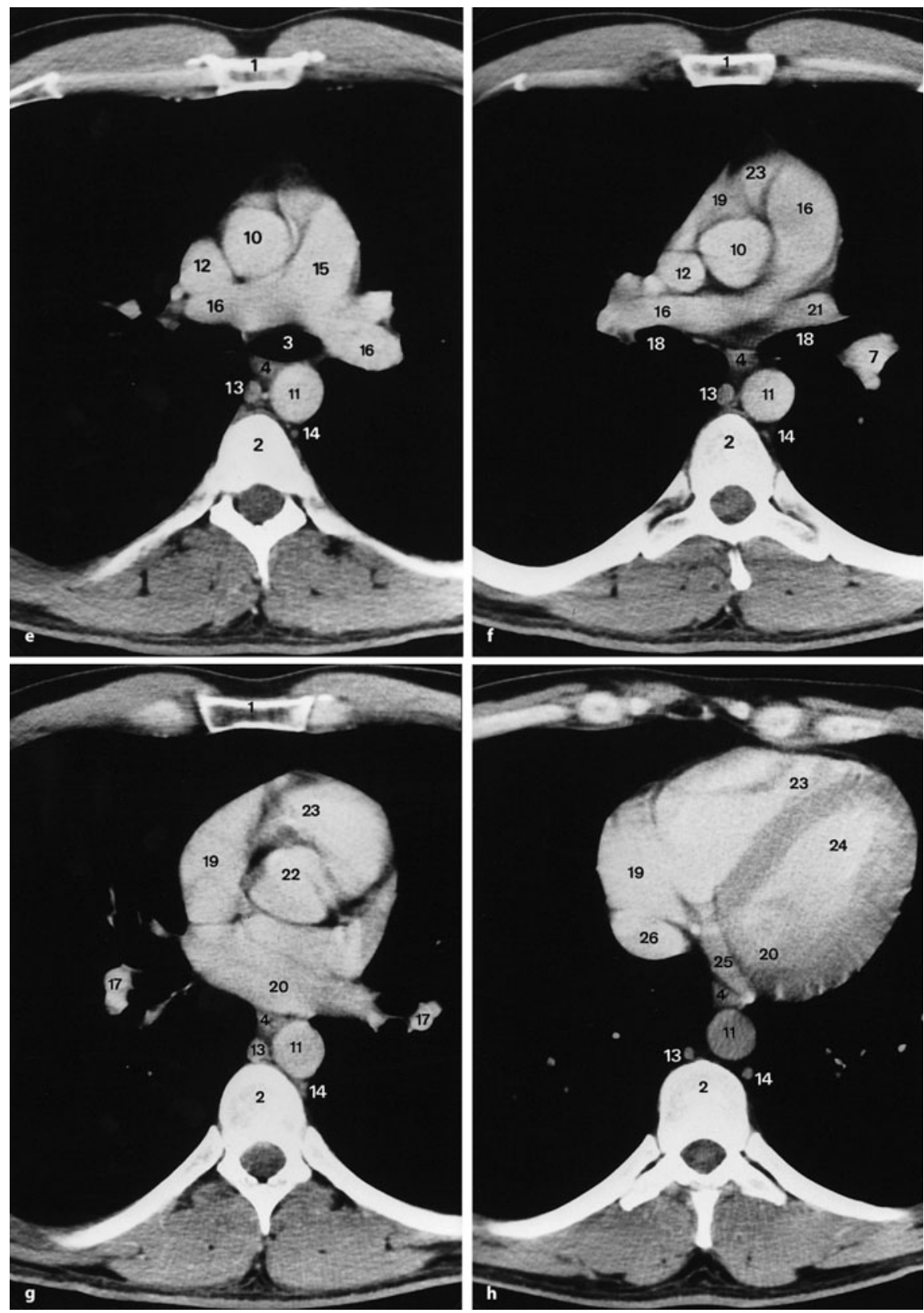

Abb. $17.3 \mathrm{e}-\mathrm{h}$. Legende s. S. 4 

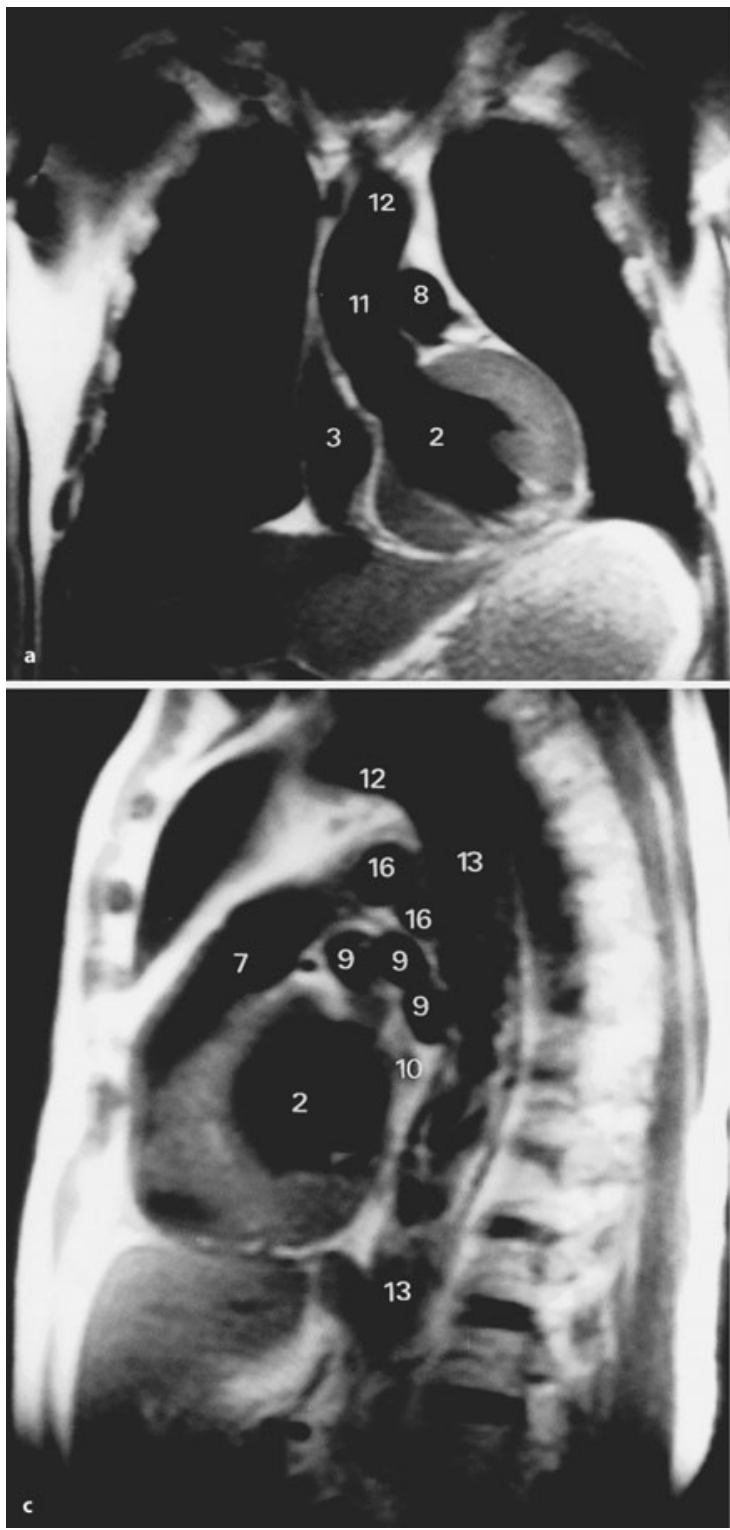

Abb. 17.4a-d. Topographische Anatomie des Mediastinums in der MR-Tomographie. (Mit freundlicher Genehmigung von Herrn Dr. B. Wintersperger, Institut für Radiologische Diagnostik, Klinikum Großhadern). 1 Rechte Herzkammer, 2 linke Herzkammer, 3 rechter Vorhof, 4 linker Vorhof, 5 V. cava

azygoösophagealen Rezessus. Die Einmündung der rechten V. mammaria interna in die V. cava superior ist - insbesondere bei Kollateralkreisläufen - an der rechten Mediastinalkontur erkennbar. Die V. hemiazygos liegt nach Durchtritt durch das Zwerchfell prävertebral im linken hinteren Mediastinum und entgeht, wie die $V$. hemiazygos accessoria, im Normalfall der bildlichen Darstellung. Der Begriff ,aortopulmonales Fenster" bezeichnet eine zwischen Aor-
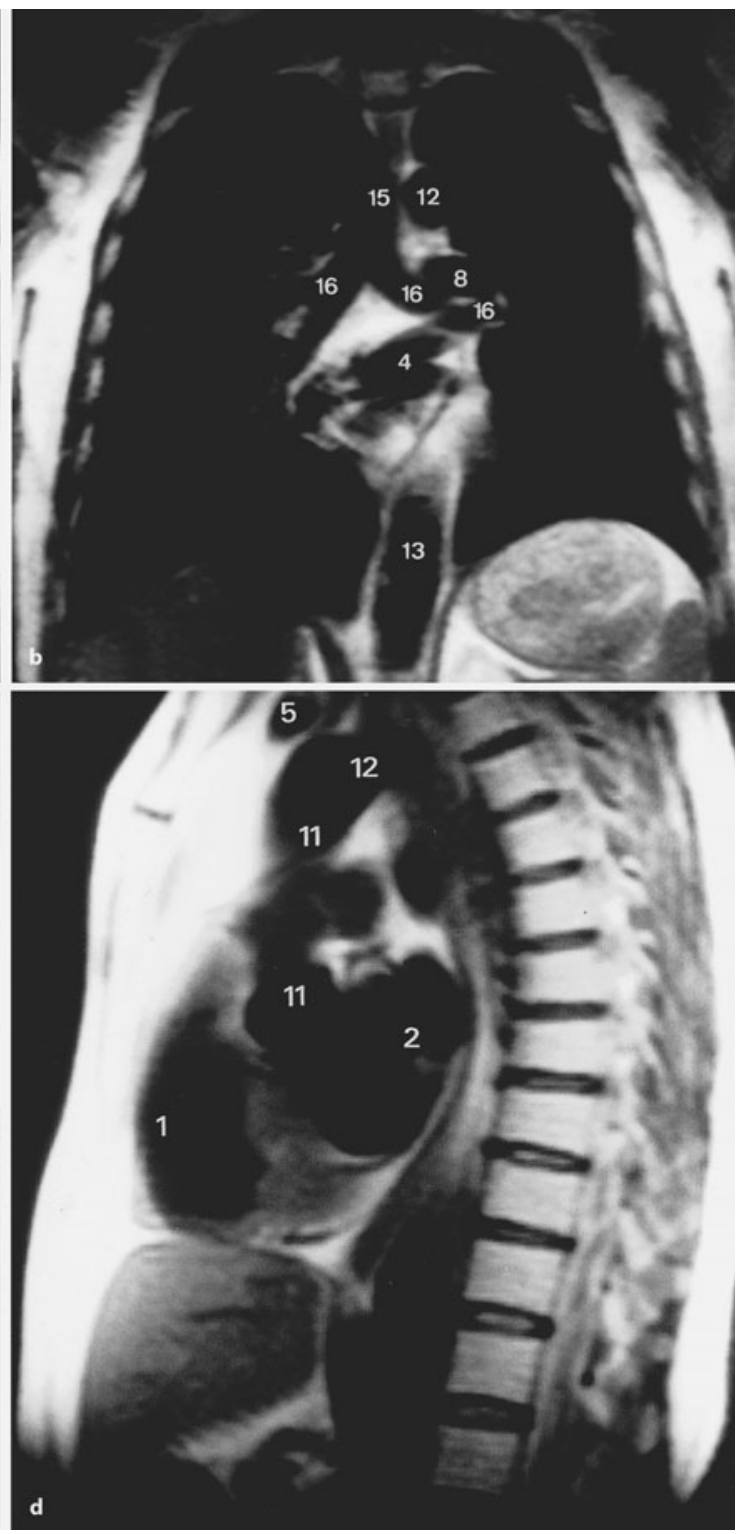

superior, $6 \mathrm{~V}$. cava inferior, 7 Truncus pulmonalis, $9 \mathrm{~A}$. pulmonalis dextra bzw. sinistra, 10 Lungenvene, 11 Aorta ascendens, 12 Aortenbogen, 13 Aorta descendens, 14 Truncus brachiozephalicus, 15 Trachea, 16 Stammbronchus

tenbogen und Truncus pulmonalis gelegene Mediastinalnische. Ihre Weite hängt von der Elongation der Aorta und dem Kaliber der Pulmonalgefäße ab.

Die Distanz zwischen laterodorsaler Trachealwand und Lunge beträgt in der Regel $4 \mathrm{~mm}$. Das Tracheallumen ist im Bereich der Pars membranacea abgeflacht. Die Knorpelspangen der zentralen Atemwege können Kalkeinlagerungen aufweisen. Der Ösophagus ist häufig durch seinen Luftgehalt und/oder 

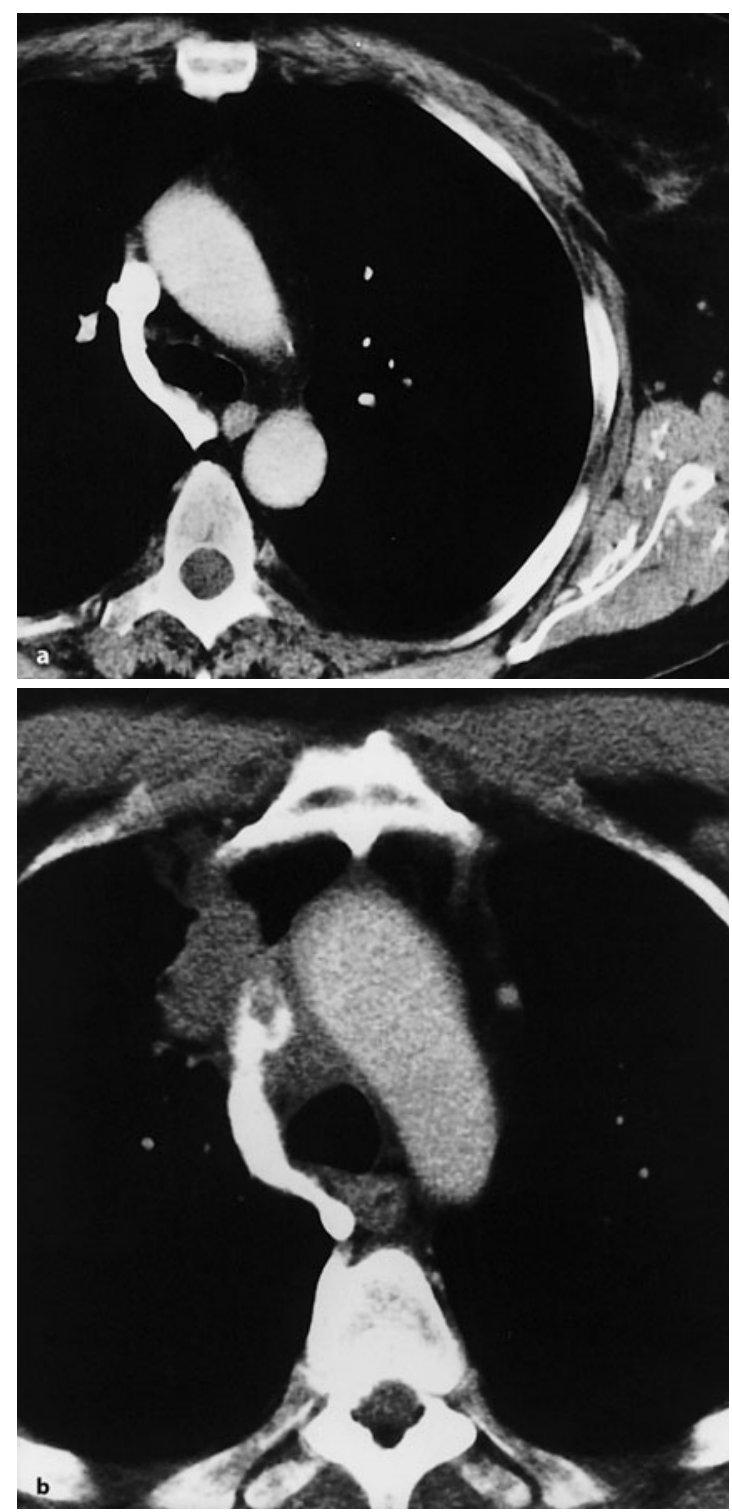

Abb. 17.5 a, b. Computertomographische Darstellung der V. azygos. a Verschluss der linken V. brachiocephalica mit Abfluss des links cubital injizierten Kontrastmittels über die V. azygos in die V. cava superior. b Thrombose der V. cava superior an der Einmündung der V. azygos bei einem Patienten mit mediastinalem Non-Hodgkin-Lymphom

peroral gegebenes, röntgenpositives Kontrastmittel markiert. Er liegt im oberen Mediastinum der Trachea dorsal an, nimmt kaudal der Trachealbifurkation einen links gerichteten Verlauf und überkreuzt supradiaphragmal die Aorta descendens. Eine Wanddicke von $3 \mathrm{~mm}$ gilt als normal. Nerven und Ductus thoracicus sind in der Regel nicht erkennbar. Ihre Lage kann nur relativ zu den Nachbarstrukturen vermutet werden. Lediglich die $\mathrm{Nn}$. phrenici sind gele- gentlich punktförmig an den lateralen Konturen des Herzens sichtbar.

Die mediastinalen Faszien bestimmen analog der retroperitonealen Verhältnisse die Ausbreitung hämorrhagischer und exsudativer Prozesse. Ösophagus und Trachea sind von lockerem Fett-/Bindegewebe umgeben, das von der periviszeralen Faszie eingescheidet wird. Dieser periviszerale Raum kommuniziert nach kranial mit dem Larynx, Trachea und Pharynx beinhaltenden zervikalen Kompartiment, das von der prätrachealen und buccopharyngealen Faszie begrenzt wird. Nach kaudal setzt sich der periviszerale Raum entlang der Bronchien bis in die Lungenperipherie fort und kommuniziert mit dem subepikardialen Fettgewebe. Die periviszerale Faszie ist mit der periaortalen Adventitia bindegewebig verbunden. Die prävertebrale Faszie reicht von der Schädelbasis bis zum Kreuzbein und umgibt das paravertebrale Fett-/Bindegewebe ventrolateral. Sie stellt eine Barriere für die ventrolaterale Ausbreitung vertebraler und paravertebraler entzündlicher Prozesse dar und erklärt deren kraniokaudale Ausbreitungstendenz.

Die Lymphknoten des Mediastinums werden unter topographischen Gesichtspunkten zu Gruppen zusammengefasst. Im vorderen Mediastinum werden die dorsal der Rippenknorpel lokalisierten parasternalen (Mammaria-interna-)Lymphknoten von den prävaskulären Lymphknoten unterschieden, die vereinzelt retrosternal (perikardial), in der Hauptsache jedoch ventral der großen Gefäße (Aorta ascendens, V. cava superior, Truncus brachiocephalicus, V. jugularis interna, V. subclavia) liegen. Sie dienen dem Lymphabfluss aus den medialen Mammaanteilen, der vorderen Thoraxwand und den medialen Anteilen der Leberkapsel. Im mittleren Mediastinum treten die an der unteren Zirkumferenz des Perikards und an den Ligamenta pulmonalia lokalisierten parietalen Lymphknoten gegenüber den viszeralen Lymphknoten zahlenmäßig zurück. Letztere werden entsprechend ihrer Lokalisation in paratracheale, tracheobronchiale, bifurkale (subcarinale) und bronchopulmonale Lymphknoten unterschieden. Im hinteren Mediastinum kommunizieren die ventral der Rippenköpfchen gelegenen interkostalen Lymphknoten mit den entlang des unteren Ösophagus und der Aorta descendens lokalisierten posterioren mediastinalen Lymphknoten.

Je nach Ortsauflösung des gewählten bildgebenden Verfahrens und Fettgehalt des umgebenden Gewebes sind mediastinale Lymphknoten ab einem Querdurchmesser von 3-5 mm erkennbar (Abb. 17.6; Leppert 1998; Schaefer-Prokop 1998; Wegener 1992). Computertomographisch grenzen sie sich als weichteilisodense Strukturen vom umgebenden hypodensen mediastinalen Fettgewebe (ca. -10 bis -80 H.E.) 


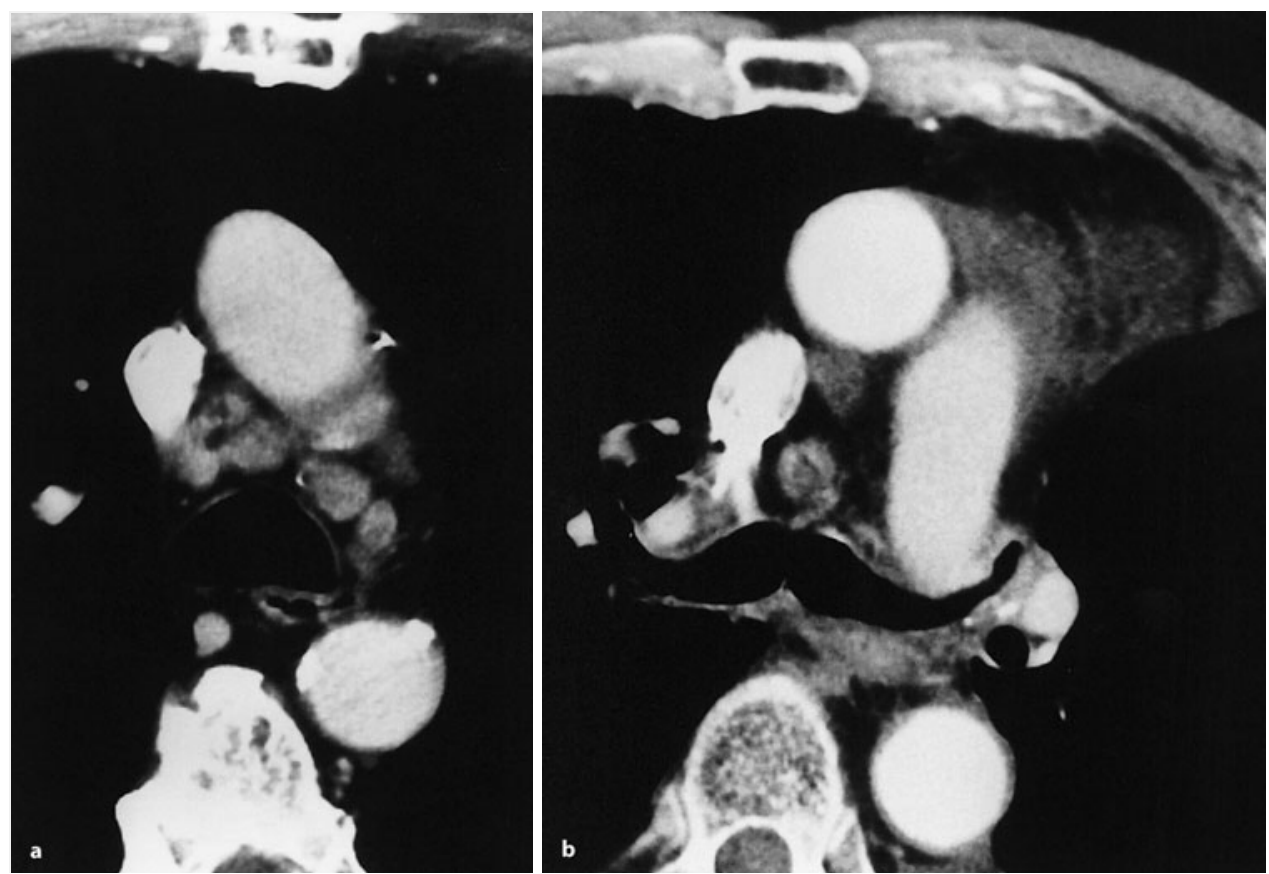

Abb. 17.6 a, b. Computertomographische Darstellung von zentral verfetteten und daher benignen mediastinalen Lymphknoten. a Bei einem Patienten mit malignem Melanom
Stadium T1N1M0. b Bei einem Patienten mit linksseitigem Pleura- und Perikarderguss ab. MR-tomographisch sind sie durch ihre intermediäre Signalintensität vom sowohl in T1-als auch in T2-Wichtung signalreichen Mediastinalfett differenzierbar. Nach intravenöser Kontrastmittelgabe zeigen normale Lymphknoten einen mäßigen, homogenen Kontrastanstieg. Sie besitzen eine ovaläre Form, wobei die Längsachse meist parallel zu den vaskulären Leitstrukturen ausgerichtet ist. Das Verhältnis von Längs- zu Querdurchmesser liegt normalerweise $\geqq 2$, der Querdurchmesser $<10 \mathrm{~mm}$. Er wird in anatomischen Arbeiten im Mittel mit 3-6 mm angegeben. Nur bei den tracheobronchialen und bifurkalen Lymphknoten werden in Folge der erhöhten Drainagefunktion physiologische Querdurchmesser von 11-12 mm beobachtet.

In axialer Schichtführung bieten Trachea, Aorta ascendens, Aorta descendens, V. cava superior, Ösophagus und V.azygos gut beurteilbare Queranschnitte. Geringe Volumenzunahmen der benachbarten Lymphknoten werden daher vergleichsweise leicht erfasst. Wegen ihres zu Partialvolumeneffekten führenden schrägen bis horizontalen Verlaufs bieten Stammbronchien, Conus pulmonalis und zentrale Pulmonalgefäße ungünstigere Abbildungsverhältnisse. Das aortopulmonale Fenster ist erst bei einem Abstand $>1,5 \mathrm{~cm}$ zwischen Aorta und Truncus pulmonalis sicher zu beurteilen. Der spitzwinklige azygoösophageale Rezessus wird durch bifurkale (sub- carinale) Lymphknotenvergrößerungen laterokonvex verformt. Die komplexe Gefäß- und Bronchialstruktur des Hilus setzt zur computertomographischen Beurteilung geringer Lymphknotenvergrößerungen eine intravenöse Kontrastmittelgabe voraus.

Der Thymus ist ein ventral der Aorta ascendens im vorderen Mediastinums gelegenes, paarig angelegtes Organ (Abb. 17.7). Der linke Flügel ist meist größer als der rechte und kann bis in das aortopulmonale Fenster reichen. In zwei Dritteln der Fälle sind beide Thymuslappen zu einer dreieckigen Gewebeformation verschmolzen, seltener finden sich zwei oder nur ein isolierter Thymuslappen. Als Teil des lymphatischen Systems hat der Thymus bei Neugeborenen sein größtes Volumen (Daldrup 1998; Kushihashi 1996; Merten 1991; Schaefer-Prokop 1998; Siegel 1996; Wegener 1992). Im Laufe der Kindheit und Adoleszenz bildet er sich kontinuierlich zurück. Die Dicke der Thymuslappen ist ein besseres $\mathrm{Ma} ß$ als ihre Länge. Sie sollte bis zum 20. Lebensjahr $1,8 \mathrm{~cm}$ und später $1,3 \mathrm{~cm}$ nicht überschreiten. Die computertomographische Dichte des Thymus entspricht in Kindheit und Jugend der der Muskulatur, mit zunehmender Involution sinkt sie zunehmend ab und wird nach dem 40. Lebensjahr fettisodens. Die Organinvolution kann gleichmäßig oder herdförmig erfolgen. Im Endstadium findet sich eine fibröse Organmatrix. MR-tomographisch hat Thymusgewebe in 


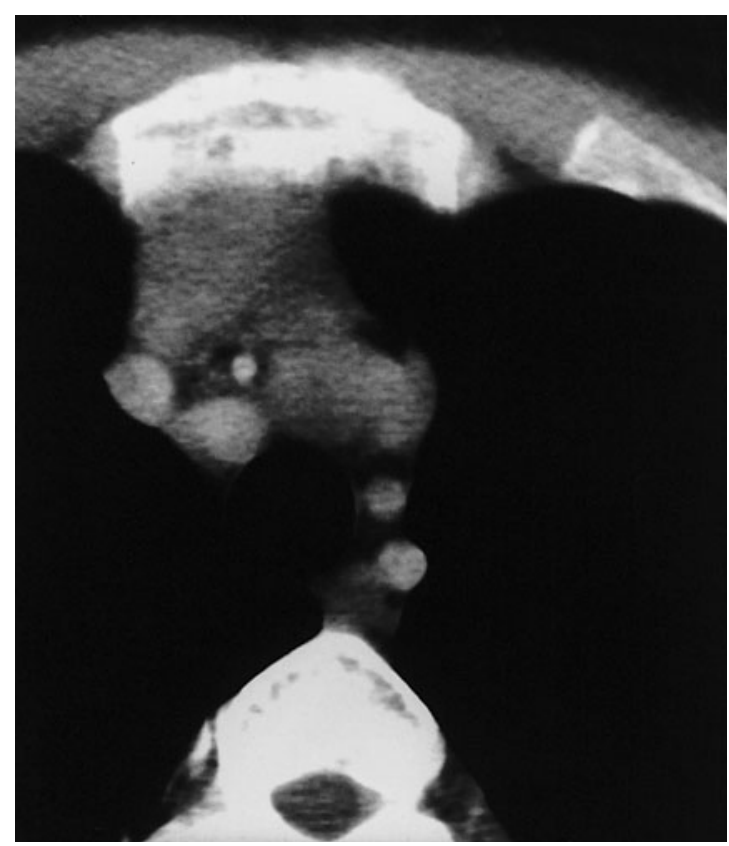

Abb. 17.7. Normaler Thymus eines 7-Jährigen

Tabelle 17.1. Häufigkeit mediastinaler Primärtumoren und tumorähnlicher Läsionen $(\mathrm{n}=1893)$. (Mod. nach Hofman $\mathrm{u}$. Otto 1991 sowie Anyanwu u. Krysa 1991)

\begin{tabular}{lcc} 
Tumor & n & [\%] \\
\hline Maligne Lymphome & 382 & 20 \\
Thymogene Tumoren & 321 & 17 \\
Schilddrüsentumoren & 297 & 16 \\
Mediastinale Zysten & 229 & 12 \\
Neurogene Tumoren & 167 & 9 \\
Teratome/Keimzelltumoren & 145 & 8 \\
Sonstige maligne Tumoren & 141 & 7 \\
Sonstige tumorähnliche Läsionen & 113 & 6 \\
Sonstige benigne Tumoren & 98 & 5
\end{tabular}

der Jugend in allen Wichtungen eine niedrigere Signalintensität als Fettgewebe, mit zunehmender Involution gleicht sich die Signalintensität des Parenchyms der des umgebenden Fettgewebes an.

\section{2}

\section{Fehlbildungen}

\section{Pathologie}

Bei den meisten zystischen Mediastinaltumoren (10-27\% aller primären Mediastinalgeschwülste) handelt es sich um kongenitale Fehlbildungen des Bronchialbaums und des neuroenteralen Kanals (Tabelle 17.1, 17.2; Aktogu 1996; Anayanwu u. Krysa 1991; Anawanyu 1991; Haddon 1991; Hofmann 1991; Kohmann 1993; Lange 1996; Levasseur 1976; Merten 1991; Schaefer-Prokop 1998; Wegener 1992).

Bronchogene Zysten stellen etwa $50 \%$ aller kongenitalen Mediastinaltumoren (Tabellen 17.1, 17.317.6). Entwicklungsgeschichtlich handelt es sich um Aussprossungen aus dem Tracheobronchialsystem, die ihren Anschluss an das Tracheobronchialsystem verloren haben. In ca. 30\% liegen bronchogene Zysten in Nachbarschaft der zentralen (mediastinale Lokalisation) und in ca. $70 \%$ in Nachbarschaft der peripheren Atemwege (pulmonale Lokalisation). Bei paratrachealer Lage werden sie auch als tracheobronchiale Zysten bezeichnet. Mediastinale bronchogene Zysten sind in der Regel in Umgebung der Trachealbifurkation lokalisiert. Ist die fibröse Verbindung zu den zentralen Atemwegen verloren, so finden sich bronchogene Zysten auch paraösophageal, in der Ösophaguswand, retrosternal und im hinteren Mediastinum. Pathologisch-anatomisch handelt es sich um kugelförmige Raumforderungen, deren Wandaufbau histologisch dem eines Bronchus entspricht. Sie sind mit einem Sekret von seröser bis stark visköser Konsistenz gefüllt. Selten besteht ein Anschluss an das Tracheobronchialsystem.

Tabelle 17.2. Topographie mediastinaler Tumoren $(n=742)$. (Mod. nach Levasseur et al. 1976)

\begin{tabular}{|c|c|c|c|c|}
\hline & & $\begin{array}{l}\text { Vorderes Mediastinum } \\
{[\%]} \\
69\end{array}$ & $\begin{array}{l}\text { Mittleres Mediastinum } \\
{[\%]} \\
13\end{array}$ & $\begin{array}{l}\text { Hinteres Mediastinum } \\
{[\%]} \\
20\end{array}$ \\
\hline Oberes Mediastinum & $48 \%$ & $\begin{array}{l}\text { Schilddrüsentumoren } \\
\text { Lymphome } \\
\text { Teratome } \\
\text { Thymustumoren }\end{array}$ & $\begin{array}{l}\text { Schilddrüsentumoren } \\
\text { Bronchogene Zysten }\end{array}$ & $\begin{array}{l}\text { Schilddrüsentumoren } \\
\text { Neurogene Tumoren }\end{array}$ \\
\hline Mittleres Mediastinum & $33 \%$ & $\begin{array}{l}\text { Thymustumoren } \\
\text { Teratome }\end{array}$ & Bronchogene Zysten & Neurogene Tumoren \\
\hline Unteres Mediastinum & $19 \%$ & $\begin{array}{l}\text { Lipome } \\
\text { Pleuroperikardzysten } \\
\text { Thymustumoren }\end{array}$ & Bronchogene Zysten & Neurogene Tumoren \\
\hline
\end{tabular}


Tabelle 17.3. Primäre und sekundäre mediastinale Raumforderungen. (Mod. nach Anyanwu u. Krysa 1991)

Primäre Mediastinaltumoren

Neurogene Tumoren

Keimzelltumoren

Thymome

Lymphome

Endokrine Tumoren: Struma, Nebenschilddrüsenadenom, Karzinoid

Zystische Tumoren: bronchogene Zyste, Perikardzyste, enterogene Zyste, Thymuszyste

\section{Sekundäre Mediastinaltumoren}

Metastasen aller malignen Tumoren

Ösophagustumoren

Substernale Strumen

Aortenaneurysmata

Hiatushernie, Morgagni-Hernie

Anteriore Meningozele

Pankreaspseudozyste

Akzessorische Milz

Tabelle 17.4. Zystische Mediastinaltumoren. (Mod. nach Amyamwu 1991)

\begin{tabular}{lll} 
Angeboren & Erworben & Parasitär \\
\hline Bronchogen & Hernien & Echinokokkus \\
Ösophageal & D. thoracicus & \\
Gastroenteral & Pankreaspseudozyste & \\
Tracheoösophageal & Neoplastisch & \\
Perikardial & Teratodermoid & \\
Thymogen & Lymphangiom & \\
Mesothelzyste & Zystisches Thymom & \\
Meningozele & &
\end{tabular}

Tabelle 17.5. Klassifikation thymogener bzw. mediastinaler Tumoren und tumorähnlicher Läsionen. (Mod. nach Hofmann u. Otto 1991)

Thymogene Mediastinaltumoren

Thymome

Thymuskarzinoide

Mesenchymale Thymustumoren

Thymolipome

Thymushyperplasie

Histiozytäre Tumoren

Maligne Lymphome

M. Hodgkin

Non-Hodgkin-Lymphome

Tumorähnliche Läsionen

Thymogene Zysten

Thymushyperplasie

\section{Nicht thymogene Mediastinaltumoren}

Keimzelltumoren/Teratome

Histiozytäre Tumoren

Maligne Lymphome

M. Hodgkin

Non-Hodgkin-Lymphome

Neurogene Tumoren

Tumorähnliche Läsionen

Mediastinale Zysten

Angiofollikuläre Lymphknotenhyperplasie

Tabelle 17.6. Staging epithelialer Thymustumoren. (Nach Masaoka et al. 1981)
Stadium I
Makroskopisch allseits kapselbegrenzte Thymome, histologisch keine Kapselinfiltration
Stadium II
Makroskopisch Invasion des parathymalen Fettgewebes und/oder der mediastinalen Pleura,
Stadium III histologisch Kapselinfiltration
Stadium IVa
Makroskopische Infiltration benachbarter Organe wie Perikard, Lunge und Gefäße
Stadium IVb
Pleurale und/oder perikardiale Tumorinfiltration
Lymphatische oder hämatogene Metastasen 
Angeborene tracheobronchiale Fisteln sind sehr selten. Sie sind histologisch aus unterschiedlichen Tracheal- und Ösophaguswandanteilen aufgebaut und nehmen kontinuierlich an Größe zu, bis sich schließlich eine zystische Raumforderung entwickelt hat.

Aus dem dorsalen Anteil des Urdarms entwickeln sich Ösophagus und Gastrointestinaltrakt. Alle zystischen Fehlbildungen, die sich aus diesem Anteil des Urdarms entwickeln, werden unter dem Begriff „enterale Zysten“ zusammengefasst, Synonyme sind „Duplikationszysten“, „Anschlusszysten“ und „Duplikaturen“. Ösophageale und gastroenterale Zysten beruhen auf einer fehlerhaften Umwandlung der in der Embryonalzeit soliden Speiseröhre in ein Hohlorgan. Sie sind in Umgebung des Ösophagus (ösophageale Zysten) und im hinteren Mediastinum anzutreffen (gastroenterale Zysten). Enterale Zysten sind mit Ösophagus-, Magen- oder Dünndarmepithel ausgekleidet. Sie enthalten ein wässriges Sekret und im Falle eines Anschlusses an den Magen-DarmTrakt häufig Luft.

Neuroenterale Zysten entstehen durch eine unvollständige Trennung des Endoderms von der notochordalen Platte. Im Gegensatz zu bronchogenen Zysten sind neuroenterale Zysten paravertebral lokalisiert und sind eine Differentialdiagnose zu Ductusthoracicus-Zysten, die typischerweise erst bei einer Sektion diagnostiziert werden. Neuroenterale Zysten sind in der Regel von Ösophagus-, Magen- oder Dünndarmepithel ausgekleidet und über einen Stiel sowohl mit den Meningen als auch mit unterschiedlichen Abschnitten des Gastrointestinaltraktes verbunden.

Das Perikard entsteht aus der Verschmelzung ursprünglich nicht miteinander in Verbindung stehender lakunärer Hohlräume. Werden bei der embryonalen Verschmelzung einzelne Lakunen ausgespart, so entstehen Perikardzysten, die bevorzugt im rechten, aber auch im linken kardiophrenischen Winkel auftreten. Pathologisch-anatomisch handelt es sich um mono- bis polyzystische Raumforderungen, die von kubischen oder flachen Epithelzellen ausgekleidet sind und seröse Flüssigkeit enthalten. Pleuroperikardiale Zysten sind meist zwischen 3 und $8 \mathrm{~cm}$ groß. Sie kommunizieren nur selten mit der Perikardhöhle.

Mesothelzysten entwickeln sich aus der gleichen Anlage wie Perikardzysten. Sie befinden sich in anderen Lokalisationen des Mediastinums und sind nicht mit dem Perikard verbunden.

Angeborene Thymuszysten sind auf eine Entwicklungsstörung der dritten Kiementasche zurückzuführen.

Meningozelen (s. Abschn. 16.4.2) gehören im engeren Sinne nicht $\mathrm{zu}$ der Gruppe der angeborenen Mediastinalzysten, sollten jedoch in die Differential- diagnose zystischer Raumforderungen des hinteren Mediastinums einbezogen werden.

\section{Klinik}

Angeborene Mediastinalzysten sind meist asymptomatisch und werden im frühen Erwachsenenalter als Zufallsbefund auf einer Thoraxübersichtsaufnahme diagnostiziert (Aktogu 1996; Anayanwu u. Krysa 1991; Anawanyu 1991; Benzarti 1997; Haddon 1991; Kohmann 1993; Lange 1996; Merten 1991; SchaeferProkop 1998). Durch Kompression paraspinaler Nerven, der zentralen Atemwege und der Speiseröhre können bei größeren Raumforderungen Schmerzen, Luftnot und Erbrechen auftreten. Bronchogene Zysten, die in Umgebung der Carina lokalisiert sind, führen meist schon in der Kindheit zu Obstruktionserscheinungen. Paraösophageale Zysten haben gelegentlich eine Dysphagie zur Folge, werden jedoch nur selten superinfiziert. Einblutungen und Infektionen können zu plötzlicher starker Größenzunahme führen. In seltenen Fällen kann eine bronchogene Zyste obliterieren. Bei gastroenteralen Zysten kann Magensekret durch Andauung der Zystenwand gastritische Beschwerden und im Extremfall eine Zystenperforationen mit entsprechenden Komplikationen verursachen. Enterale Zysten sind typischerweise mit weiteren Fehlbildungen wie Spina bifida, Skoliose, Hemivertebrae, Meningozele, Herzmissbildungen, intraabdominellen Zysten und Malrotationen des Darms vergesellschaftet.

\section{Projektionsradiographie}

Auf der Thoraxübersichtsaufnahme stellen sich angeborene Mediastinalzysten als rundlich-ovaläre, meist glatt konturierte mediastinale Raumforderungen dar (Abb. 17.8; Aktogu 1996; Anayanwu u. Krysa 1991; Anawanyu 1991; Haddon 1991; Kohmann 1993; Lange 1996; Merten 1991; Murray 1992). Bronchogene Zysten sind typischerweise in unmittelbarer Nachbarschaft der Atemwege gelegen (Abb.17.9 a, b). Enterale Zysten sind vorwiegend im hinteren Mediastinum lokalisiert und können gelegentlich Kalzifizierungen aufweisen. Luft-Flüssigkeits-Spiegel weisen auf eine Verbindung zu den zentralen Atemwegen oder dem Gastrointestinaltrakt hin. Durch perorale Kontrastierung der Speiseröhre lässt sich eine Verlagerung oder Lumeneinengung des Ösophagus diagnostizieren. Eine glatt begrenzte Raumforderung im kardiophrenischen Winkel macht eine Perikardzyste wahrscheinlich (Abb.17.10). Ossäre Missbildungen wie Spina bifida, Skoliose und Hemivertebrae lassen an mediastinale Missbildungstumoren denken. Eine sichere diagnostische Zuordnung ist jedoch bildmorphologisch nicht möglich und bedarf bei therapeutischer Relevanz der histologischen Klärung. 

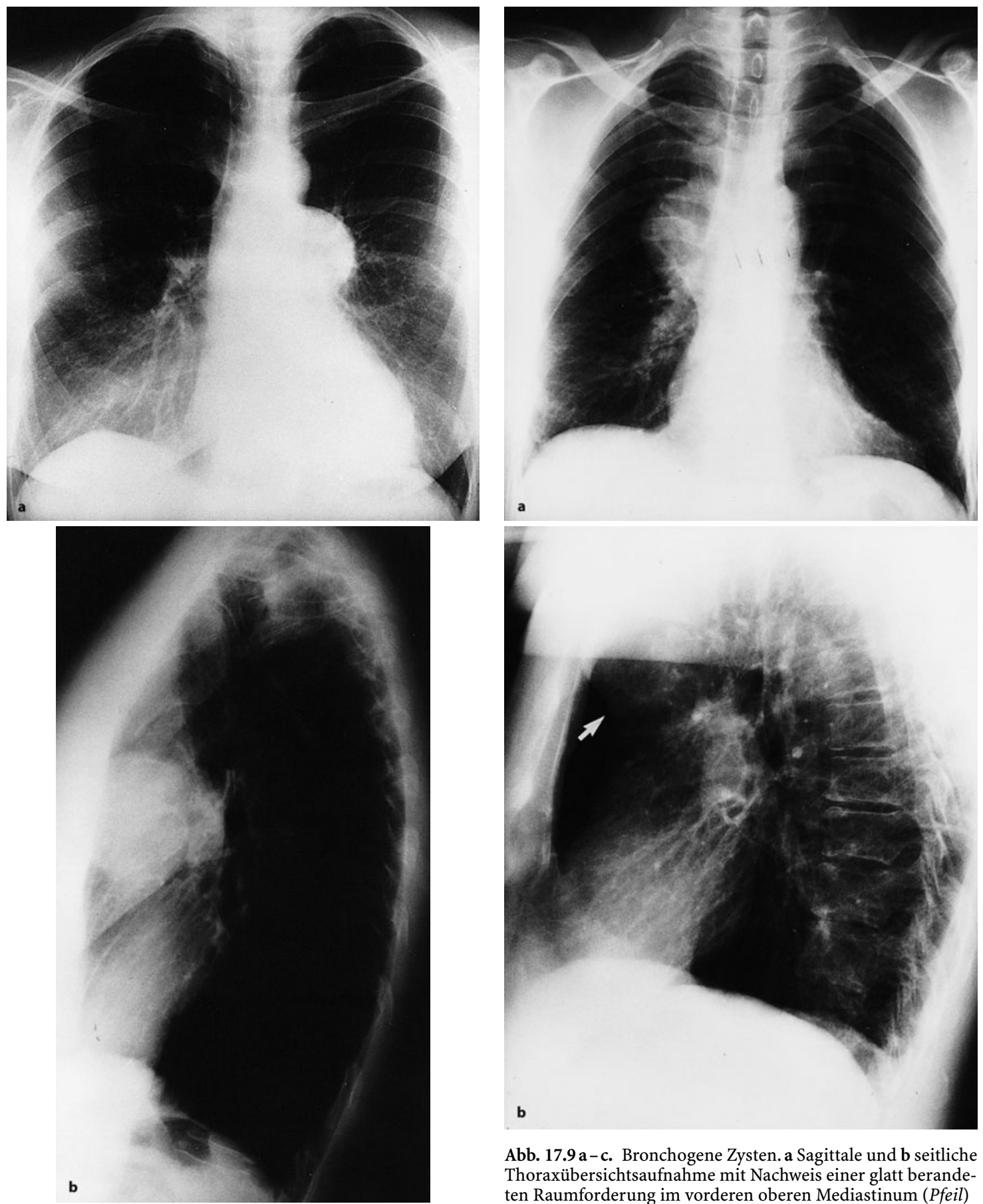

Abb. 17.9 a-c. Bronchogene Zysten. a Sagittale und b seitliche Thoraxübersichtsaufnahme mit Nachweis einer glatt berandeten Raumforderung im vorderen oberen Mediastinum (Pfeil)

Abb. 17.8 a, b. Verkalkte mediastinale Zyste. a Sagittale und b seitliche Thoraxübersichtsaufnahme 


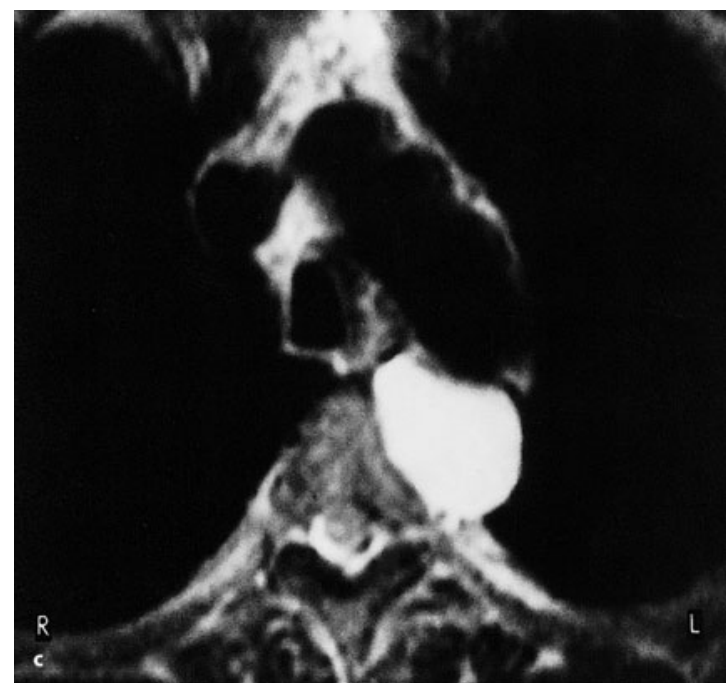

Abb. 17.9c. T2-gewichtetes Turbo-Spin-Echo-Bild mit Nachweis einer signalreichen bronchogenen Zyste im hinteren oberen Mediastinum (anderer Patient)

\section{Schnittbilddiagnostik}

Schnittbilddiagnostisch lässt sich die die zystische Natur der Raumforderung und ihre topographische Beziehung zu den Nachbarstrukturen klären (Aktogu 1996; Anayanwu u. Krysa 1991; Anawanyu 1991; Bittner 1998; Daldrup 1998; Haddon 1991; Kohmann 1993; Lange 1996; Lesko 1999; Merten 1991; Murray 1992; Schaefer-Prokop 1998; Siegel 1991; Wegener 1992; Wilson 1994). Es handelt sich in der Regel um dünnwandige Zysten von variabler Form. Die Dichte der Flüssigkeit schwankt je nach Eiweißgehalt zwischen -5 und $25 \mathrm{H}$. E. bei pleuroperikardialen Zysten (Abb. 17.10b) und zwischen 20 und 50 H.E. bei bronchogenen und enteralen Zysten, sodass eiweißreiche Zysten computertomographisch als solide Prozesse fehlgedeutet werden können. Die MRTomographie ist der Computertomographie wegen der eindeutigeren Differenzierung von Zysten mit proteinreichem Inhalt und soliden Geweben methodisch überlegen (Abb.17.9c). MR-tomographisch stellt sich die Zystenflüssigkeit unabhängig von ihrem Eiweißgehalt in T2-Wichtung signalreich dar, wohingegen in T1-Wichtung seröse Flüssigkeiten signalarm und eiweißreiche Flüssigkeiten isointens bis signalreich zur Abbildung kommen. Nach intravenöser Kontrastmittelgabe findet sich computerund MR-tomographisch kein intrazystisches Enhancement, sondern allenfalls ein Enhancement in der Zystenwand.

Bronchogene Zysten liegen in über 50\% der Fälle rechts parakarinär, seltener paratracheal, paraösophageal und retrokardial. Bei paravertebraler Lage kommt eine enterale Zyste in Betracht. Pleuroperi-
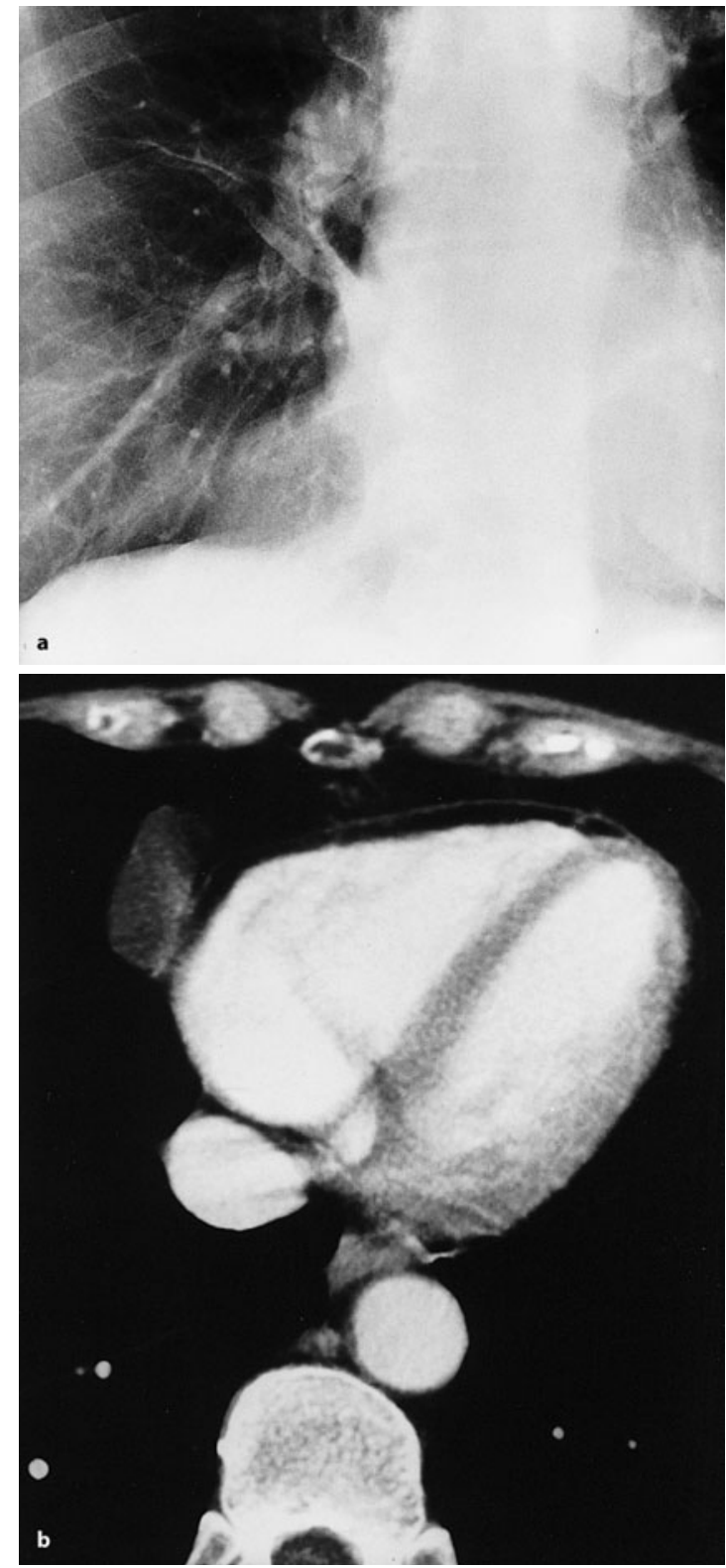

Abb. 17.10 a, b. Perikardzyste. a Ausschnittsvergrößerung einer Thoraxübersichtsaufnahme, b CT nach intravenöser Kontrastmittelgabe

kardiale Zysten sind typischerweise in den vorderen kardiophrenischen Winkeln lokalisiert. Luft-/Flüssigkeitsspiegel weisen auf einen Anschluss an das Tracheobronchialsystem oder den Gastrointestinaltrakt hin.

Enterale Zysten sind häufiger mit Malformationen der Wirbelsäule wie Hemivertebrae, Schmetterlingswirbel und Skoliose vergesellschaftet. Die Abgrenzung gegenüber mediastinalen Pankreaspseudozys- 
ten bereitet wegen der meist eindeutigen Anamnese und Klinik sowie dem obligaten infradiaphragmalen Befund keine Schwierigkeiten. Die Artdiagnose ist jedoch in der Regel nicht zu stellen.

\section{3}

\section{Entzündungen}

\subsection{1}

\section{Akute Mediastinitis}

\section{Pathologie}

Entzündliche Mediastinalerkrankungen werden in akute und chronische Formen unterteilt (Tabelle 17.1). Die akute Mediastinitis ist Folge einer bakteriellen Infektion und nimmt typischerweise einen foudroyanten, häufig letalen Verlauf (Kohmann 1993; Lange 1996; Schaefer-Prokop 1998; Wegener 1992). Sie beruht in über $90 \%$ der Fälle auf einer Ösophagusperforation durch iatrogene Manipulation (Endoskopie, Biopsie, Fremdkörperextraktion), Tumorinfiltration oder äußere Verletzung. Spontane Ösophagusperforationen werden vereinzelt nach schwerem Erbrechen (Boerhaave-Syndrom) beobachtet und sind in der Regel suprakarinär lokalisiert. Seltener sind akute Mediastinitiden Folgen einer Operation (Sternotomie, Magenhochzug) oder zervikaler Entzündungen, die sich entlang der präformierten Faszienräume in das Mediastinum ausbreiten. Entzündungen der Pleura, der Lunge und der Wirbelsäule greifen aufgrund der anatomisch-topographischen Gegebenheiten erst spät auf das Mediastinum über. Das entzündliche Exsudat durchsetzt die mediastinalen Spalträume in der Regel diffus. Seltener bilden sich mediastinale Abszesse, die ihrerseits in die Speiseröhre, die zentralen Atemwege und die Pleurahöhlen einbrechen können.

\section{Klinik}

Plötzlich auftretende schwere retrosternale Schmerzen, die in die Halsweichteile ausstrahlen, Fieber, Schüttelfrost, eine obere Einflussstauung und ein zervikales Weichteilemphysem lassen an eine akute Mediastinitis denken.

\section{Projektionsradiographie}

Auf der Thoraxübersichtsaufnahme zeigt sich eine fortgeschrittene Mediastinitis als unscharf begrenzte, bilaterale, meist kranial betonte Mediastinalverbreiterung (Abb.17.11 a; Lackner 1998; Lange 1996; Murray 1992). Der Vergleich mit Voraufnahmen kann bei entsprechender Klinik zur Bewertung initialer Veränderungen hilfreich sein. Lufteinschlüsse in Projektion auf das mittlere Mediastinum und die Halsweichteile können Folge einer Ösophagusperforation
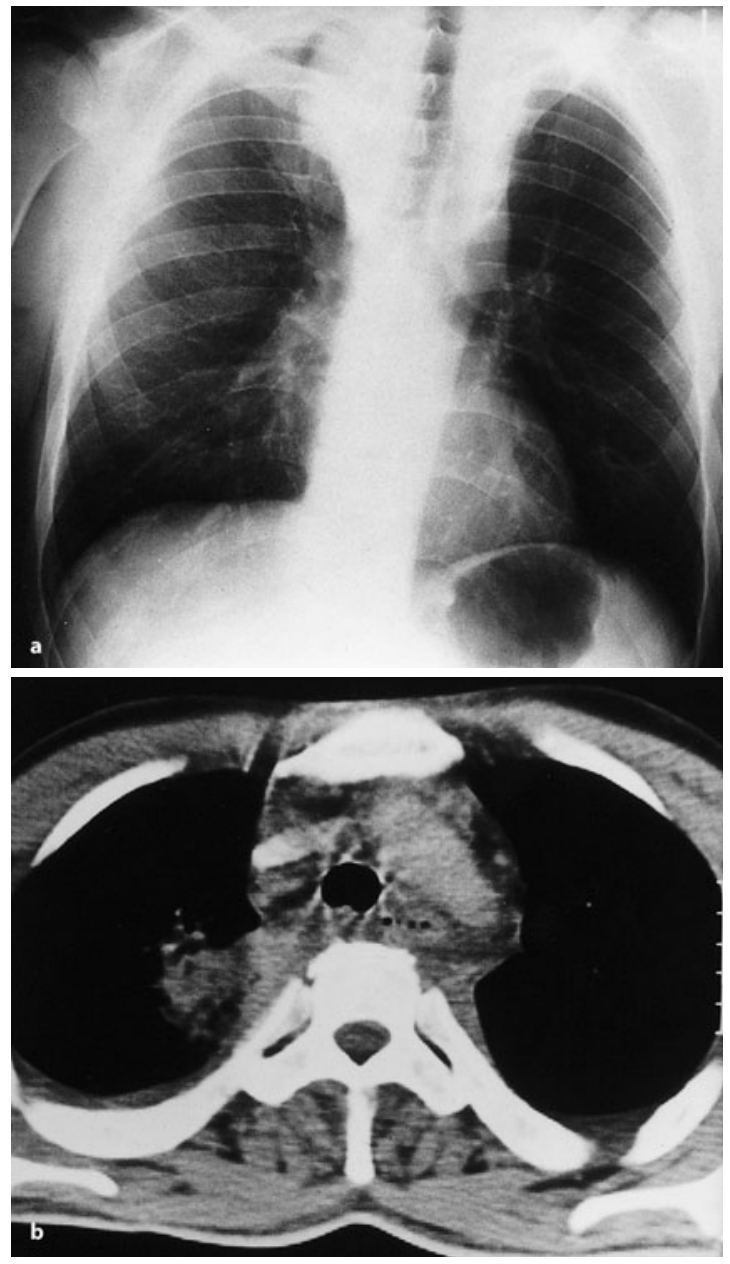

Abb. 17.11 a, b. Akute Mediastinitis infolge einer iatrogenen Ösophagusperforation: a Die Thoraxübersichtsaufnahme zeigt eine glatt begrenzte Verbreiterung des oberen Mediastinums. b Computertomographisch sind extraluminale Lufteinschlüsse und eine diffuse Dichteanhebung des mediastinalen Fett-/Bindegewebes durch das entzündliche Ödem nachzuweisen

sein. Oft werden begleitende Pleuraergüsse, gelegentlich ein meist links lokalisierter Seropneumothorax beobachtet. Bei entsprechendem Verdacht ist die Durchführung einer Ösophagusbreischluckuntersuchung indiziert. Mediastinale Gasansammlungen, die nicht auf eine Ösophagusperforation oder eine Operation zurückzuführen sind, sind als Ausdruck einer Abszedierung zu werten.

\section{Schnittbilddiagnostik}

Über die Aussage der Thoraxübersichtsaufnahme hinausgehend zeigt das mediastinale Fett- und Bindegewebe als Ausdruck der entzündlichen Exsudation eine streifige bis diffuse Anhebung der Dichte (CT) 


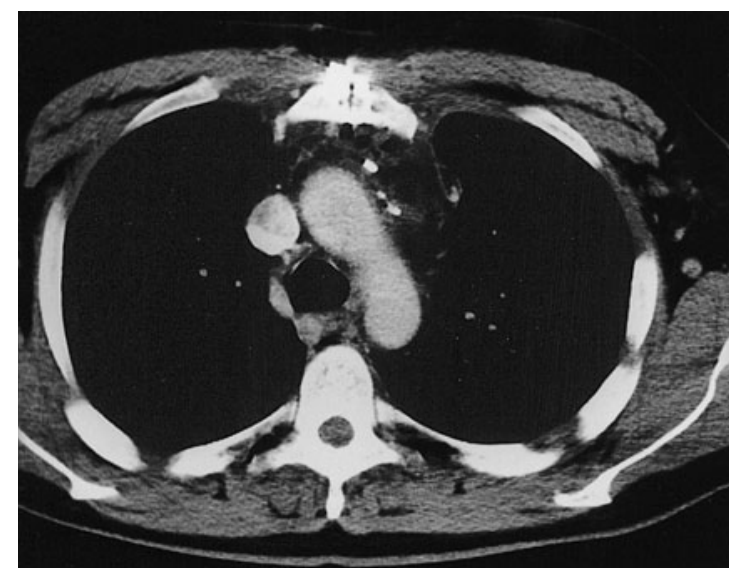

Abb. 17.12. Computertomographische Darstellung physiologischer mediastinaler Lufteinschlüsse nach Herzoperation

bzw. der Signalintensität (T2-gewichtetes MR-Bild; Abb.17.11b; Bittner 1998; Lange 1996; Lesko 1999 Murray 1992; Schaefer-Prokop 1998; Siegel 1996; Wegener 1992; Wilson 1994). Bei schlanken Patienten ist der Abstand zwischen den Mediastinalgefäßen durch das Exsudat vergrößert. Luftansammlungen lassen sich einzelnen mediastinalen Kompartimenten und Organstrukturen zuordnen, wodurch die Differentialdiagnose eingeengt wird. Umschriebene Flüssigkeitsansammlungen mit hyperdensem/hyperintensem Randsaum nach intravenöser Kontrastmittelgabe machen insbesondere bei Vorliegen zentraler Lufteinschlüsse eine Abszedierung wahrscheinlich (s. Abschn. 17.4.5). In der postoperativen Phase ist $\mathrm{zu}$ bedenken, dass kleinere Luft- und Flüssigkeitsansammlungen in der Regel bis zu 20 Tage und selten bis zu 50 Tage nach der Operation nachweisbar sind, ohne dass eine Entzündung vorliegt (Abb. 17.12). Erst ein Wiederauftreten oder eine $\mathrm{Zu}$ nahme in Verlaufskontrollen deuten auf ein entzündliches Geschehen hin.

\subsection{2}

\section{Chronische Mediastinitis}

\section{Pathologie}

Die Ätiologie der chronischen Mediastinitis ist heterogen und zum Teil ungeklärt (Tabelle 17.1). Meist ist sie infektiösen Ursprungs (Tuberkulose, Mykosen, Histoplasmose, Aktinomykose, Syphilis), sie kann jedoch auch als Folge eines Mediastinalhämatoms, einer Bestrahlung, einer medikamentösen Therapie (Methysergid) oder eines mediastinalen M. Ormond sein (Hainaut 1998; Kohman 1993; Lange 1996; Schaefer-Prokop 1998; Wegener 1992).
Eine Unterscheidung in granulomatöse und fibrosierende Formen ist nicht sinnvoll, da eine granulomatöse Mediastinitis schleichend in eine fibröse Mediastinitis übergehen kann und sich die Röntgenmorphologie beider Entitäten nicht unterscheidet. Die chronische Mediastinitis betrifft nahezu ausnahmslos das obere Mediastinum im paratrachealen, subcarinalen und hilären Bereich. Die Diagnose einer chronischen Mediastinitis wird in der Regel histologisch gestellt. Pathologisch-anatomisch zeigen sich Veränderungen im Rahmen einer granulomatösen Entzündung oder eine derbe Bindegewebsplatte, die die großen Mediastinalgefäße, die zentralen Atemwege und den Ösophagus einmauert.

\section{Klinik}

Der klinische Verlauf ist in der Regel subakut. Bei der tuberkulösen Mediastinitis im Kindesalter sind ausgeprägte Lymphknotenschwellungen typisch, die Kompressionserscheinungen hervorrufen können. Die entzündeten Lymphknoten können einschmelzen und Anschluss an die Atemwege gewinnen. Bei Erwachsenen sind tuberkulöse Lymphknotenvergrößerungen nur mäßig ausgeprägt (Hainaut 1998; Lange 1996; Moon 1996; Schaefer-Prokop 1998). Das gleichzeitige Vorliegen einer retroperitonealen Fibrose, einer Riedel-Struma oder eines orbitalen Pseudotumors spricht für eine idiopathische fibröse Mediastinitis. Ein Verschlusssyndrom der V. cava superior wird in etwa $10 \%$ der Fälle, also seltener als bei neoplastischen Mediastinalprozessen beschrieben. Gelegentlich wird eine Dysphagie durch Einengungen des Ösophagus oder eine Dyspnoe durch Einengung der zentralen Atemwege und der zentralen Pulmonalgefäße beobachtet.

\section{Projektionsradiographie}

Die radiologischen Symptome variieren mit der Ausdehnung und Lokalisation der chronischen Entzündung bzw. Fibrosierung. Die Thoraxübersichtsaufnahme kann einen mediastinalen Normalbefund, eine meist das obere Mediastinum betreffende diffuse Verbreiterung oder eine lobulierte, meist rechts betonte, paratracheale Raumforderung zeigen. Verkalkungen sind selten. Pulmonale Infiltrate und hiläre Lymphknotenvergrößerungen können auf die Genese der Erkrankung hindeuten (Kohman 1993; Lange 1996; Murray 1992). Pulmonale Minderbelüftungen aufgrund einer Obstruktion der zentralen Atemwege, eine pulmonal-venöse Stauung aufgrund einer Obstruktion der zentralen Lungenvenen oder eine pulmonal-arterielle Hypertonie aufgrund einer Obstruktion der zentralen Lungenvenen oder der zentralen Pulmonalarterien legen in solchen Fällen die Diagnose einer chronischen Mediastinitis nahe. 


\section{Schnittbilddiagnostik}

Die chronische Mediastinitis findet ihre computertomographische Entsprechung in einer meist im oberen mittleren Mediastinum gelegenen Verdichtung, die die mediastinalen Fettschichten maskiert und den Ösophagus, die zentralen Atemwege und die großen Gefäße in unterschiedlichem Maße einscheidet (Kohman 1993; Lange 1996; Murray 1992; Schaefer-Prokop 1998; Wegener 1992; Wilson 1994). Sie kann eine flächige pseudotumoröse oder eine retikuläre Struktur aufweisen. Gelegentlich liegen nur minimale perivaskuläre Verdichtungen des mediastinalen Fettgewebes vor. MR-tomographisch wird die Signalintensität von dem Exsudatgehalt des pathologisch veränderten Gewebes bestimmt. Chronisch fibrosierende Formen weisen sowohl in T1-als auch in T2-Wichtung eine Signalintensitätsminderung im Vergleich zum gesunden mediastinalen Fett-/Bindegewebe auf (Bittner 1998; Lesko 1999; Siegel 1996). Die Intensität des Enhancements nach intravenöser Kontrastmittelgabe spiegelt die Floridität des entzündlichen Prozesses wieder. Typischerweise liegen Lymphknotenvergrößerungen vor, die von entzündlichen Lymphknotenveränderungen anderer Genese nicht zu differenzieren sind. Bei älteren Verschlüssen der V. cava superior lässt sich das Ausmaß der Thrombose anhand des Kollateralkreislaufes über das Azygosvenensystem abschätzen.

\section{4}

\section{Mediastinale Raumforderungen}

\subsection{1}

\section{Allgemeines}

\section{Pathologie}

Der topographische Begriff „Mediastinaltumor“ beinhaltet eine Vielzahl pathogenetisch und histologisch unterschiedlicher Tumoren, die mehrheitlich gutartig sind (Tabelle 17.4-17.6; Anayanwu u. Krysa 1991; Anayanwu 1991; Chang 1994; Hofmann 1991; Kohman 1993; Lackner1998; Lange 1996; Levasseur 1976; Merten 1991; Schaefer-Prokop; Wegener 1992). Die differentialdiagnostische Zuordnung orientiert sich einerseits an der Tumorlokalisation im vorderen, mittleren oder hinteren Mediastinum und andererseits an der Bildmorphologie (solide vs. zystische Tumoren, Tumorkalzifikationen, Fettanteile). Entsprechend der anatomischen Topographie sind als erworbene Tumoren im vorderen Mediastinum Thymome bzw. Thymuskarzinome (Tabelle 17.6), benigne und maligne teratogene Tumoren und maligne Lymphome $\mathrm{zu}$ erwarten. Raumforderungen im mittleren Mediastinum gehen vom Herzen (Aneurysmen), von den großen Gefäßen (Aneurysmen, Hämatome), von der Speiseröhre (Ösophaguskarzinom, Dilatation des Ösophagus bei Achalasie und Sklerodermie), von den zentralen Atemwegen (Bronchialkarzinom) und von den regionären Lymphknoten (benigne Lymphadenopathie, maligne Lymphome, Lymphknotenmetastasen) aus. In Zwerchfellnähe können abdominelle Organe durch Zwerchfelllücken in das mittlere Mediastinum hernieren (axiale und paraösophageale Hiatushernie, Upside-down-Magen, Morgagni-Hernie, Bochdalek-Hernie). Raumforderungen des hinteren Mediastinums sind überwiegend neurogenen (Neurinome, Neurofibrome) oder vaskulären Ursprungs (Aneurysmen des Aortenbogens und der Aorta descendens).

Aus systematischer Sicht werden kardiovaskuläre Raumforderungen, Ösophagusprozesse und neurogene Tumoren in den Bänden "Kardiovaskuläres System“, „Gastrointestinales System“ und „Kopf Hals" dieser Reihe ausführlicher behandelt. Im Weiteren wird nur auf ihre differentialdiagnostische Bedeutung eingegangen.

\section{Klinik}

Mediastinale Raumforderungen sind meistens asymptomatisch und werden als Zufallsbefund entdeckt (Anayanwu u. Krysa 1991; Anawanyu 1991; Chang 1994; Freitas 1994; Higgins 1993; Kohman 1993; Lange 1996; Masaoka 1981; Merten 1991; SchaeferProkop 1998; Schulman 1998; Wegener 1992). Thoraxschmerzen, Husten, Dyspnoe und Fieber sind Leitsymptome eines malignen Mediastinaltumors. Eine Infiltration oder Kompression des N. recurrens führt zu Heiserkeit, des Ganglion stellatum zum HornerSyndrom und der Lymphbahnen zum mediastinalen Lymphstau und evtl. Chylothorax. Eine akute, d.h. schnell entstandene Kompression der V. cava superior hat eine obere Einflussstauung zur Folge, wohingegen eine chronische partielle oder vollständige Blockade der V. cava superior zur Ausbildung eines venösen Kollateralkreislaufs führt. Hierfür sind in $25 \%$ der Fälle benigne und in $75 \%$ der Fälle maligne Tumoren wie vom rechten Oberlappen ausgehende Bronchialkarzinome, maligne Strumen und Thymome als ursächlich zu nennen. Rückenschmerzen können Folge eines Tumors im hinteren Mediastinum sein. Eine Myasthenia gravis kann mit einem Thymom, eine paroxysmale Hypertonie mit einem neuroendokrinen Tumor (Phäochromozytom, chromaffines Paragangliom, Ganglioneurom) und eine Hyperkalzämie mit einem solitären Adenom (85\%), multiplen Adenomen (4\%), einer Hyperplasie (10\%) oder einem Karzinom (1\%) der Nebenschilddrüse vergesellschaftet sein.

Die Aufgaben der bildgebenden Diagnostik bei der Abklärung mediastinaler Raumforderungen bestehen in der Identifizierung des Tumors und seiner 
Abb. 17.13 a, b.

Durchleuchtungsgesteuerte Zielaufnahmen der Trachea während des MüllerManövers (Erzeugung eines intrathorakalen Unterdrucks, a) und des Valsalva-Manövers (Erzeugung eines intrathora-kalen Überdrucks, b).

Links betonte, retrosternale Struma mit Trachealverlagerung nach rechts. Keine Trachealinstabilität
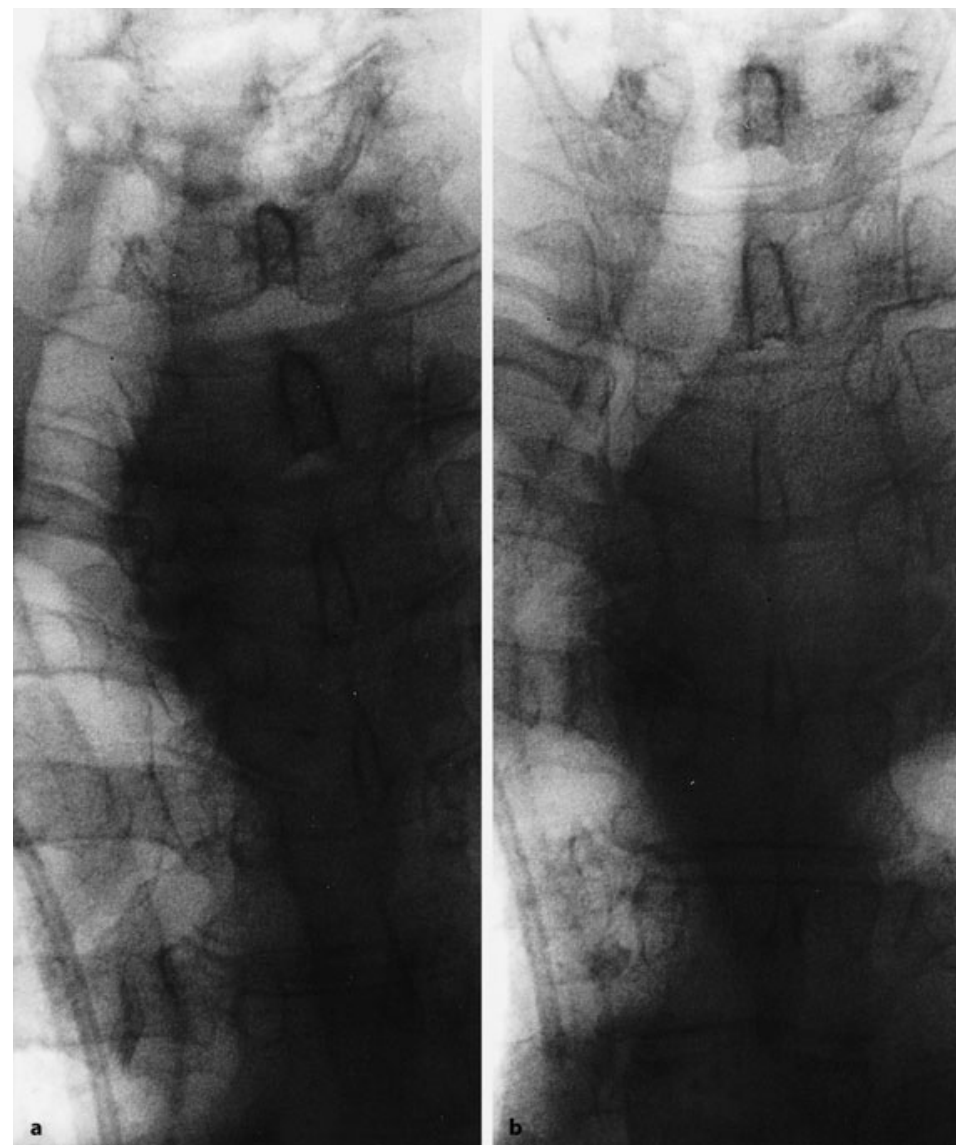

Lokalisation, in der Beschreibung der Tumormorphologie, in der Erfassung von pulmonalen, pleuralen oder ossären Begleiterkrankungen, in der Einengung der Differentialdiagnose und in der Therapiekontrolle. Hierbei ist schnelles, klinisch effizientes und kosteneffektives Vorgehen von Bedeutung.

\section{Projektionsradiographie}

Die Thoraxübersichtsaufnahme in posterior-anteriorem und seitlichem Strahlengang stellt die Basisdiagnostik des Mediastinums dar (Anayanwu u. Krysa 1991; Anawanyu 1991; Chang 1994; Cole 1995; Kohman 1993; Lackner 1998; Lange 1996; Merten 1991; Murray 1992; O’Donovan). Sie erlaubt die Beurteilung von Krankheitsverläufen und hat eine große differentialdiagnostische Breite (Herz, Lunge, Mediastinum, Skelett). Mit Thoraxübersichtsaufnahmen lassen sich Mediastinaltumoren ab einem Durchmesser von etwa $1,5 \mathrm{~cm}$ zuverlässig nachweisen. Kleinere Tumoren entgehen dem projektionsradiographischen Nachweis, da sie von den physiologischen Thoraxstrukturen überlagert werden. Tho- raxübersichtsaufnahmen geben keine zuverlässige Auskunft über die Dichte der mediastinalen Raumforderung. Nur Kalkeinschlüsse sind aufgrund des großen Absorptionsunterschiedes sicher von Weichteilgewebe $\mathrm{zu}$ unterscheiden. Durch perorale Gabe eines röntgenpositiven Kontrastmittels können Lage und Wandkonturen des Ösophagus dargestellt werden. Soweit Schluckstörungen im Vordergrund stehen, sollte ein Ösophagusbreischluck erfolgen. Die Stabilität der Trachealwand wird durch durchleuchtungsgesteuerte Zielaufnahmen während der Ausführung des Müller- (Erzeugung eines intrathorakalen Unterdrucks) und des Valsalvamanövers (Erzeugung eines intrathorakalen Überdrucks) kontrolliert (Abb. $17.13 \mathrm{a}, \mathrm{b})$. Die konventionelle Tomographie hat ihre Bedeutung durch die Einführung der digitalen Schichtbildverfahren CT und MRT verloren. Zur prätherapeutischen Darstellung des topographischen Bezugs eines Mediastinaltumors zu den großen Mediastinalgefäßen kann eine Arterio- oder Kavographie notwendig werden. Die Myelographie wurde in weiten Indikationsbereichen durch die MR-Tomographie ersetzt. 


\section{Schnittbilddiagnostik}

Wegen ihrer überlagerungsfreien Darstellung der Pathoanatomie und ihres überlegenen Weichteilkontrastes werden die Computertomographie und in letzter Zeit zunehmend auch die MR-Tomographie (Abb. 17.14) zur Beschreibung der genauen Lokalisation und Morphologie projektionsradiographisch nachgewiesener Mediastinalprozesse, zum präoperativen TMN-Staging und zur Suche nach kleineren, auf der Thoraxübersichtsaufnahme mutmaßlich nicht sichtbaren mediastinalen Raumforderungen bei entsprechendem klinischen Verdacht eingesetzt (Anayanwu u. Krysa 1991; Anawanyu 1991; Bittner 1998; Chang 1994; Daldrup 1998; Ferguson 1998; Higgins 1993; Kohman 1993; Lesko 1999; Merten 1991; Murray 1992; Schaefer-Prokop 1998; Siegel 1996; Storto, Wegener 1992; Wilson 1994). In der klinischen Routine hat sich wegen ihrer diagnostischen Genauigkeit und der vergleichsweise günstigen Kosten-Nutzen-Relation die Computertomographie bewährt. Bei Staging-Untersuchungen werden Sensitivitäten zwischen 75-95\%, Spezifitäten zwischen 65-100\% und Treffsicherheiten zwischen $75-95 \%$ angegeben. Die MR-Tomographie gilt in

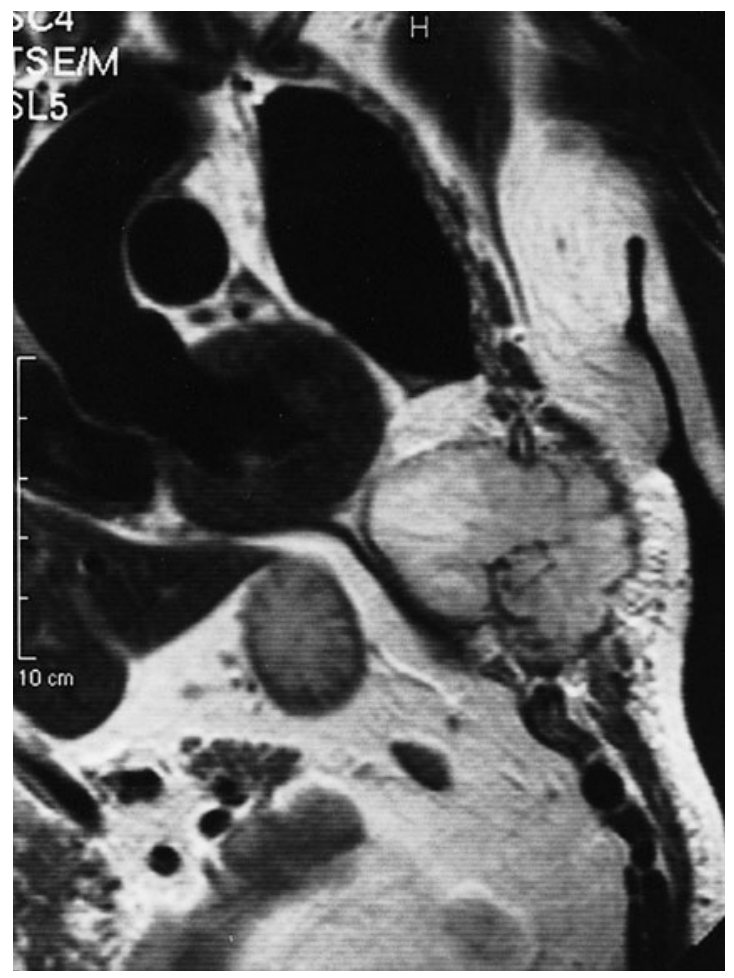

Abb. 17.14. Das Mediastinum infiltrierendes Chondrosarkom. T2-gewichtetes Turbo-Spin-Echo-Bild in der die maximale mediastinale Tumorausdehnung erfassenden angulierten Schichtrichtung der Beurteilung des Mediastinums der Computertomographie als ebenbürtig. Von Vorteil sind die fehlende Strahlenexposition, die freie Wahl der Schichtebenen, der hohe Weichteilkontrast und die von einer intravenösen Kontrastmittelgabe weitgehend unabhängige Darstellung der Gefäßstrukturen. Als nachteilig gelten die vergleichsweise hohen Kosten, die geringere Geräteverfügbarkeit, die bislang etwas niedrigere Ortsauflösung und die untersuchungstechnisch noch nicht vollständige behobene Anfälligkeit für Bewegungsartefakte. Daher sollte nur bei Fragestellungen, die das hintere Mediastinum betreffen, und bei Kindern oder Patienten mit bekannter Kontrastmittelunverträglichkeit der MR-Tomographie der Vorzug gegeben werden.

Die transkutane Sonographie erlaubt bei jugulärer und parasternaler Einschallung eine begrenzte Einsicht in das vordere obere Mediastinum (Betsch 1994). Eine retrosternale Struma ist diagnostizierbar. Eine Beurteilung der kaudalen Anteile ausgedehnterer Strumen und eine genaue topographische $\mathrm{Zu}$ ordnung eines vergrößerten Schilddrüsenlappens zu Trachea und den großen Gefäßen ist jedoch wegen der Behinderung der Schallwellenausbreitung durch Knochen und Luft nicht möglich, sodass sich die Methode bei der Klärung mediastinaler Fragestellungen nicht breiter durchsetzte.

Durch die Einführung der transösophagealen Endosonographie wurde eine sonographische Beurteilung der Ösophaguswand und des periösophagealen Mediastinums einschließlich des Herzens und der Aorta ascendens möglich (Schüder 1995). Die Präzision, mit der die anatomischen Schichten der Ösophaguswand dargestellt werden können, wird durch kein anderes bildgebendes Verfahren erreicht. Kraniokaudale Begrenzung und Infiltrationstiefe von Ösophagustumoren werden in etwa zwei Dritteln der Fälle richtig bestimmt. Ösophagusimpressionen durch mediastinale Tumoren können sicher von intramuralen Tumoren unterschieden werden.

Die genannten Verfahren erlauben nur eine begrenzte differentialdiagnostische Zuordnung mediastinaler Tumoren. Diese orientiert sich an Lokalisation, Form, Kontur und Dichte (CT) bzw. Signalintensität (MRT) des Fremdgewebes vor und nach intravenöser Kontrastmittelgabe. Mehrere, nicht operationspflichtige mediastinale Tumoren wie Thymolipome, Zysten und benigne Strumen können in Zusammensicht mit Anamnese und Klinik sicher diagnostiziert werden. In vielen Fällen ist jedoch zur Wahl des geeigneten Therapieverfahrens eine histologische Sicherung notwendig. Bei günstiger Lokalisation im vorderen oder hinteren Mediastinum bieten sich perkutane CT- oder neuerdings auch $M R$ gesteuerte Biopsietechniken zur Dignitätsklärung 
einer mediastinalen Raumforderung an. Für die histologische Beurteilung maligner Lymphome ist der Materialbedarf jedoch so groß, dass oft einer offenen Biopsie über eine Mediastinoskopie oder eine Thorakotomie der Vorzug gegeben wird. In seltenen Fällen kann die interventionelle Behandlung einer trachealen, ösophagealen oder venösen Tumorobstruktion durch Laserung und/oder Stent-Einlage sinnvoll sein.

\subsection{2}

\section{Primäre Mediastinaltumoren}

Primäre Mediastinaltumoren sind als Raumforderungen definiert, die von einer im Mediastinum gelegenen Organstruktur ausgehen (Anayanwu u. Krysa 1991; Anayanwu 1991; Chang 1994; Daldrup 1998; Kohman 1993; Lackner 1998; Lange 1996; Levasseur 1976; Merten 1991; Schaefer-Prokop 1998; Wegener 1992). Auf angeborene primäre Mediastinaltumoren wurde im Abschn. 17.2 eingegangen. Erworbene primäre Mediastinaltumoren sind selten, sie sind durch große Heterogenität ausgezeichnet (Tabellen 17.2-17.6).

Da sich die Mediastinalorgane aus allen drei Keimblattanlagen entwickeln, werden im Mediastinum neben histogenetisch unterschiedlichen Tumoren teratoide Geschwülste mit ekto-, ento- und mesodermalen Anteilen gefunden.

\section{Vorderes Mediastinum}

\section{Pathologie und Klinik}

Primärtumoren des vorderen Mediastinum gehen von den dort befindlichen parenchymalen und mesenchymalen Geweben aus (Tabelle 17.3, Anayanwu u. Krysa 1991; Anayanwu 1991; Brown 1991; Chang 1994; Hofmann 1991; Kohman 1993; Lackner 1998; Lange 1996; Masaoka 1981; Merten 1991; Saleeb 1999; Schaefer-Prokop 1998; Wegener 1992). In der Hauptsache handelt es sich um retrosternale Strumen, Thymome, Teratome und primäre Lymphknotenerkrankungen. Aber auch Perikardtumoren, Paragangliome, Lipome, Hämangiome, Hämatome oder Morgagni-Hernien kommen in Betracht. Oft ist die Erkrankung nicht auf das vordere Mediastinum beschränkt, sondern bezieht das mittlere Mediastinum ein. Bei zystischen Prozessen ist an Thymuszysten, Teratome, Dermoidzysten und Strumen zu denken. Teratome, Dermoidzysten, maligne Keimzelltumoren und Strumen weisen typischerweise Kalzifikationen auf. Fettanteile machen Lipome, Teratome oder Dermoidzysten wahrscheinlich.

Die physiologische Involution des Thymus nach dem 20. Lebensjahr geht mit dem Ersatz des Drü- senparenchyms durch Fettgewebe einher. Thymusatrophien findet man auch bei schweren Allgemeinerkrankungen und unter Chemotherapie. Reaktive Thymushyperplasien („thymic rebound“; Abb. 17.15) werden nach schweren Allgemeinerkrankungen, Radiochemotherapien, Thyreotoxikosen, HashimotoThyreoiditis, M. Addison und M. Behçet beobachtet und werden mit einer Besserung der Immunlage in Verbindung gebracht. Hierbei kann die Volumenzunahmen des Thymus über $50 \%$ des Ausgangswertes betragen. Die gleichfalls als Hyperplasie bezeichnete lymphoide Reaktion des Thymus bei Myasthenia gravis ist nicht obligat mit einer Organvergrößerung verbunden.

Primäre Thymustumoren sind selten (Tabelle 17.1). Die unter ontogenetischen und histogenetischen Aspekten komplizierte Organstruktur des Thymus hat zur Entwicklung zahlreicher Klassifikationen geführt. Mehrheitlich werden unter dem Begriff "Thymom" die epithelialen Thymustumoren subsummiert. Bei Erwachsenen ist das Thymom der häufigste Tumor im vorderen Mediastinum (Masaoka 1981). Extrem selten werden ektope Thymome im hinteren Mediastinum und im lateralen Halsbereich beschrieben. Epitheliale Thymustumoren werden meist im mittleren Lebensalter diagnostiziert. Ihre Größe schwankt von wenigen Millimetern bis $\mathrm{zu} 30 \mathrm{~cm}$. Unter klinischen und prognostischen Aspekten ist es sinnvoll, zwischen nichtinvasiven und invasiven Thymomen zu unterscheiden. Histologisch sind Thymome meistens durch mit Lymphozyten vermischte gutartig erscheinende neoplastische Thymusepithelzellen charakterisiert. Sie können eine Kapsel aufweisen, infiltrativ wachsen und intrathorakal metastasieren. Extrathorakale Absiedlungen sind eine Rarität. Thymome sind oft asymptomatisch oder aber mit Symptomen einer Myasthenia gravis assoziiert. 50\% der Patienten mit Thymom haben eine Myasthenia gravis, $10 \%$ ein paraneoplastisches Syndrom.

Maligne Thymome werden histologisch besser als Thymuskarzinome beschrieben. Thymuskarzinoide werden histogenetisch den Neuroendokrinopathien zugerechnet. Sie treten gehäuft bei Männern im mittleren Lebensalter auf und haben aufgrund ihres aggressiven Wachstums eine vergleichsweise schlechte Prognose. Primäre Thymustumoren mesenchymalen Ursprungs sind selten. Thymuslipome sind unter den mesenchymalen Thymustumoren die mit Abstand größte Gruppe. Sie treten bei Kindern und jungen Erwachsenen auf und können eine beträchtliche Größe erreichen. Erworbene Thymuszysten sind Folge einer Entzündung, einer Operation oder einer Radiochemotherapie.

Der Thymus ist bei M. Hodgkin in etwa $30 \%$ der Fälle infiltriert (Tabelle 17.1). Insofern sind im vor- 

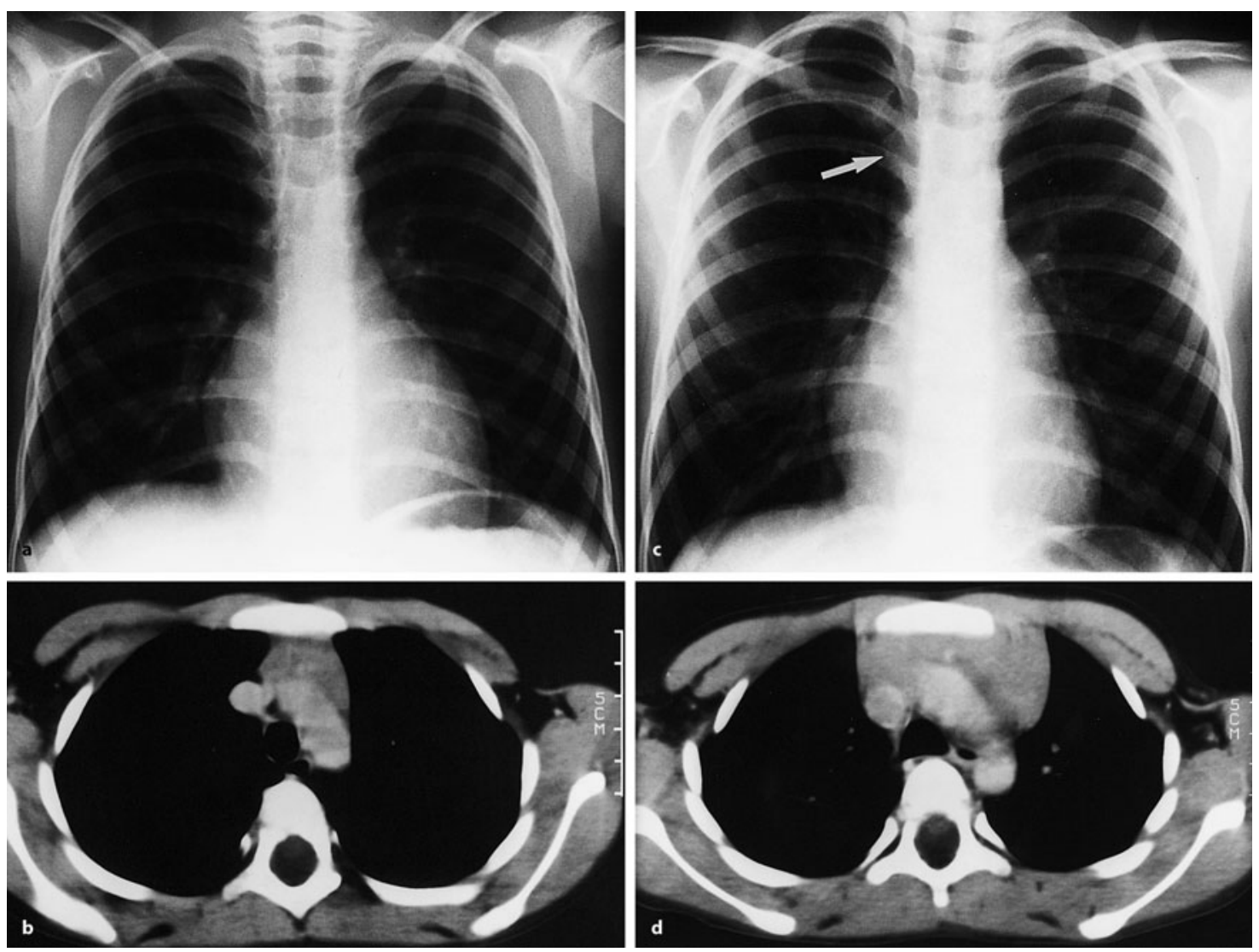

Abb. 17.15a-d. Thymushyperplasie (Rebound) nach Therapie eines Wilmstumors bei einer 4-Jährigen. a Thoraxüber-

sichtsaufnahme und b CT vor Chemotherapie. c Thoraxübersichtsaufnahme (Pfeil) und d CT nach Chemotherapie

deren Mediastinum lokalisierte maligne Lymphome wenigstens teilweise den primären Thymustumoren, überwiegend jedoch den nicht-thymischen Mediastinalgeschwülsten zuzuordnen (s. Abschn. 17.44). Unter den Non-Hodgkin-Lymphomen können sich das T-lymphoblastische Lymphom vom ,convoluted type“, das histologisch durch Zellen der Thymozytopoese gekennzeichnet ist, und das primär mediastinale hellzellige B-Zell-Lymphom, das als epithelassoziiertes Thymuslymphom interpretiert wird, als primäre Mediastinaltumoren manifestieren.

Unter dem Begriff Keimzelltumoren oder teratoide Blastome werden gut- und bösartige Teratome, Seminome (Dysgerminome), Dermoidzysten, Embryonalzellkarzinome und Chorionkarzinome zusammengefasst (Tabelle 17.1). Keimzelltumoren gehen aus Zellen der embryonalen Keimblätter hervor. Ihre histologischen Differenzierungsmuster entsprechen denen primär gonadaler Tumoren. So enthalten Teratome Zellanteile aller drei Keimblätter, während Dermoidzysten epidermalen Ursprungs sind. Das Mediastinum ist einer der häufigsten Manifestations-

orte primär extragonadaler Keimzelltumoren. Umgekehrt stellen Keimzelltumoren etwa $15 \%$ aller mediastinalen Raumforderungen dar. Das Seminom ist mit einem Anteil von ca. 30\% der häufigste mediastinale Keimzelltumor. Während für die benignen mediastinalen Keimzelltumoren keine Geschlechtsund Alterspräferenz besteht, treten maligne Formen bevorzugt bei jungen Männern auf. Zystische Tumoren erweisen sich meist als gutartig, solide eher als bösartig. In der Hälfte der Fälle finden sich Tumorverkalkungen. Analog den Verhältnissen im Retroperitoneum ist bei einer persistierenden mediastinalen Raumforderung nach Chemotherapie eines malignen Teratoms zu etwa gleichen Teilen mit vitalem Tumorgewebe, Narben bzw. Nekrosen und der Ausdifferenzierung eines „reifen“ Teratoms zu rechnen, das nur einer operativen Therapie zugänglich ist.

Nicht-thymogene mesenchymale Tumoren sind in allen drei Mediastinalkompartimenten anzutreffen (Tabelle 17.1). Die benignen Formen sind am häufigsten im vorderen, die malignen Varianten am häufigsten im hinteren Mediastinum lokalisiert. Lipome 
sind selten und meist exzentrisch gelegen. Sie können das Mediastinum kranial- und kaudalwärts verlassen und nehmen dann eine sanduhrförmige Gestalt an. Aufgrund ihrer weichen Konsistenz führen Lipome nicht zu einer Verdrängung der Nachbarorgane und stellen häufig einen Zufallsbefund dar.

Zervikale Lymphangiome und zystische Hygrome reichen in $10 \%$ der Fälle bis in das obere vordere Mediastinum (Topcu 1997). Primäre mediastinale Lymphangiome sind selten. Beide Entitäten entsprechen angeborenen, von Endothel ausgekleideten und Lymphe enthaltenden Hohlräumen, die sich nur durch ihre Durchmesser unterscheiden. Hämangiome werden gelegentlich im vorderen Mediastinum beobachtet. Bei der generalisierten Lymphangiomatose (zystische Angiomatose) liegen sowohl hämangiomatöse als auch lymphangiomatöse Anteile vor und sind selbst histologisch schwer voneinander zu differenzieren.

Eine intrathorakale Schilddrüsenvergößerung kann hyperplastisch (Jodmangel), entzündlich (Thyreoiditis) oder neoplastisch bedingt sein (Tabelle 17.1). Intrathorakale Strumaanteile gehen in zwei Dritteln der Fälle vom Isthmus und den kaudalen Polen der Schilddrüse aus und reichen in das vordere obere Mediastinum. In einem Drittel sind die dorsalen Schilddrüsenanteile hyperplastisch und dehnen sich peritracheal in das mittlere Mediastinum, oder - seltener - dorsal der Speiseröhre in das hintere Mediastinum aus.

Schilddrüsenadenome weisen häufig regressive Veränderungen mit zystischen und verkalkten Anteilen auf. Dystope Knoten sind von einer Kapsel umgeben und erhalten ihre Blutversorgung über einen Verbindungsstrang mit der Schilddrüse. Intrathorakale Strumen können die Trachea verlagern und erheblich einengen. In seltenen Fällen können benigne und maligne intrathorakale Strumen zu einer Trachealinstabilität führen. Dystope mediastinale Strumen sind selten und führen früh zu Verdrängungserscheinungen. Die Jod-123-Szintigraphie sichert durch den Nachweis von Mehrbelegungen innerhalb des Mediastinums und fehlenden Mehrbelegungen innerhalb des Schilddrüsenlagers die Diagnose.

Orthotope Nebenschilddrüsenkörperchen (ca. 90\% der Fälle) liegen den kranialen und kaudalen Schilddrüsenpolen dorsal an (Tabelle 17.1). Ektope Nebenschilddrüsen (ca. 10\% der Fälle) sind typischerweise im vorderen oberen Mediastinum, seltener im tracheo-ösophagealen Winkel gelegen. Eine Nebenschilddrüsenüberfunktion wird in $90 \%$ von Adenomen und in $10 \%$ von einer Hyperplasie hervorgerufen. Die Mehrzahl der Adenome ist hormonaktiv. Ihr Durchmesser variiert in der Regel zwischen 0,5 und $3 \mathrm{~cm}$, kann jedoch auch $10 \mathrm{~cm}$ überschreiten.
Wegen der etwa 95\%igen Erfolgsrate einer primären operativen Exploration ist eine Lokalisation ektoper Nebenschilddrüsen mittels bildgebender Verfahren nur im Falle eines postoperativ persistierenden oder rezidivierenden Hyperparathyreoidismus indiziert (Higgins 1993).Als methodische Möglichkeiten kommen in Betracht die hochauflösende Sonographie, die Technetium-Thallium-Szintigraphie, die MRTomographie, die superselektive Arteriographie und die superselektive Venographie mit Entnahme von Blutproben zur Hormonbestimmung (Cesani 1995; Freitas 1994; Saleeb 1999).

\section{Projektionsradiographie}

Auf der sagittalen Thoraxübersichtsaufnahme führen Tumoren des vorderen Mediastinums bei ausreichender Größe $\mathrm{zu}$ einer Mediastinalverbreiterung, die nicht von der Herzkontur zu trennen ist (Silhouetten-Phänomen) und im Gegensatz zu Atelektasen und Infiltraten der benachbarten Lunge die Interlobien nicht überschreitet (Abb. 17.16a, b, 17.17 a, b, 17.18 a, b; Chang 1994; Kohmann 1993; Lackner 1998; Lange 1996; Merten 1991; Murray 1992). Im Seitbild zeigt sich eine retrosternale Verdichtung, die mit der ventralen Herzkontur und der ventralen Verdichtung der Aorta ascendens konfluiert, wenn der Tumor den genannten anatomischen Leitstrukturen unmittelbar anliegt (Abb. 17.16b).

Thymustumoren projizieren sich auf der sagittalen Thoraxübersichtsaufnahme in $50-80 \%$ auf die Hilusregion (Abb.17.18 a, b). Eine niedrige Dichte, die Verwechslungsmöglichkeiten mit HerzvergröBerungen und perikardialen Zysten bietet, ist für Thymuslipome typisch. Kaudal der Klavikulaebene im vorderen oberen Mediastinum gelegene Raumforderungen mit grobscholligen Verkalkungen sind mit großer Wahrscheinlichkeit auf eine Struma zurückzuführen. Trachealverlagerung und Trachealkompression weisen auf eine Beteilgung des mittleren Mediastinums hin. Ausgedehntere Lymphangiome führen typischerweise zu Verdichtungen, die sowohl das obere vordere Mediastinum als auch die untere Zervikalregion einschließen. Auch hier ist eine Trachealverlagerung häufig. Pleuroperikardiale Fetteinlagerungen und pleuroperikardiale Zysten sind bei entsprechender Größe als glatt begrenzte, rundlich-ovaläre perikardiale Verdichtungen bevorzugt in den Herz-/Zwerchfellwinkeln erkennbar (Abb. 17.19). Hodgkin- und Non-Hodgkin-Lymphome des vorderen Mediastinums sind häufig mit Lymphomen in anderen Mediastinalkompartimenten assoziiert. Auch andere Begleitbefunde (z.B. Pleuraoder Perikardergüsse, pleurale und intrapulmonale Raumforderungen und ossäre Destruktionen) können die Differentialdiagnose einer Raumforderung im vorderen Mediastinum einengen. 



Eine Trachealinstabilität ist anhand durchleuchtungsgesteuerter Zielaufnahmen dann zu diagnostizieren, wenn sich der Querdurchmesser der Trachea in zwei senkrecht zueinander stehenden Ebenen im Müller- (intrathorakaler Unterdruck) und Valsalvamanöver (intrathorakaler Überdruck) um $>50 \%$ ändert (Abb. $17.13 \mathrm{a}, \mathrm{b}$ ).

\section{Schnittbilddiagnostik}

Ist der Tumornachweis projektionsradiographisch geführt, lässt sich die Artdiagnose durch Beschreibung der Tumorlokalisation und -morphologie mittels Computertomographie und MR-Tomographie einengen (Brown 1991; Chang 1994; Daldrup 1998; Ferguson 1998; Higgins 1993; Kushihashi 1996; Kohman 1993; Lesko 1999; Lange 1996; Merten 1991; Murray 1992; Schaefer-Prokop 1998; Siegel 1996; Wegener 1992; Wilson 1994). Aber auch kleinere me-

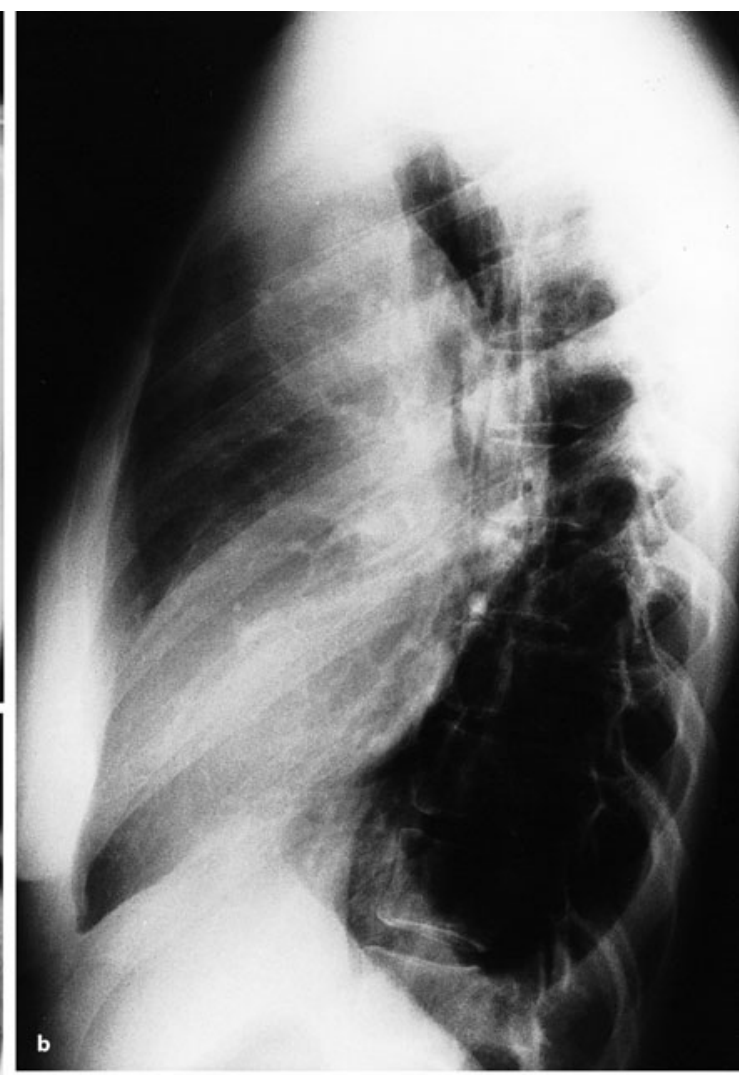

Abb. 17.16a-c. Primärtumor des vorderen Mediastinums. Histologisch handelt es sich um ein Teratom. a Thoraxübersichtsaufnahme im sagittalen und b im seitlichen Strahlengang, c CT nach intravenöser Kontrastmittelgabe

diastinale Raumforderungen, die dem projektionsradiographischen Nachweis entgehen, lassen sich computer- und MR-tomographisch diagnostizieren. Fettgewebe und flüssigkeitshaltige Zysten sind sicher von solidem Weichteilgewebe unterscheidbar. Unscharfe Randkonturen, eine fehlende Abgrenzbarkeit von Nachbarstrukturen als Zeichen der Infiltration, Lymphknotenvergrößerungen über $1,5 \mathrm{~cm}$ Querdurchmesser und ossäre Destruktionen stellen Malignitätskriterien dar.

Gelegentlich ist als Normvariante nur ein Thymuslappen angelegt, der mit einem Tumor verwechselt werden kann. In diesen Fällen behält der Lobus seine elongierte Form und legt sich den Mediastinalstrukturen an. Die physiologische Involution des Thymusparenchyms findet ihr computer- und MR-tomographisches Korrelat in zunehmend fettäquivalenten Dichtewerten bzw. Signalintensitäten (Abb. 17.7). 

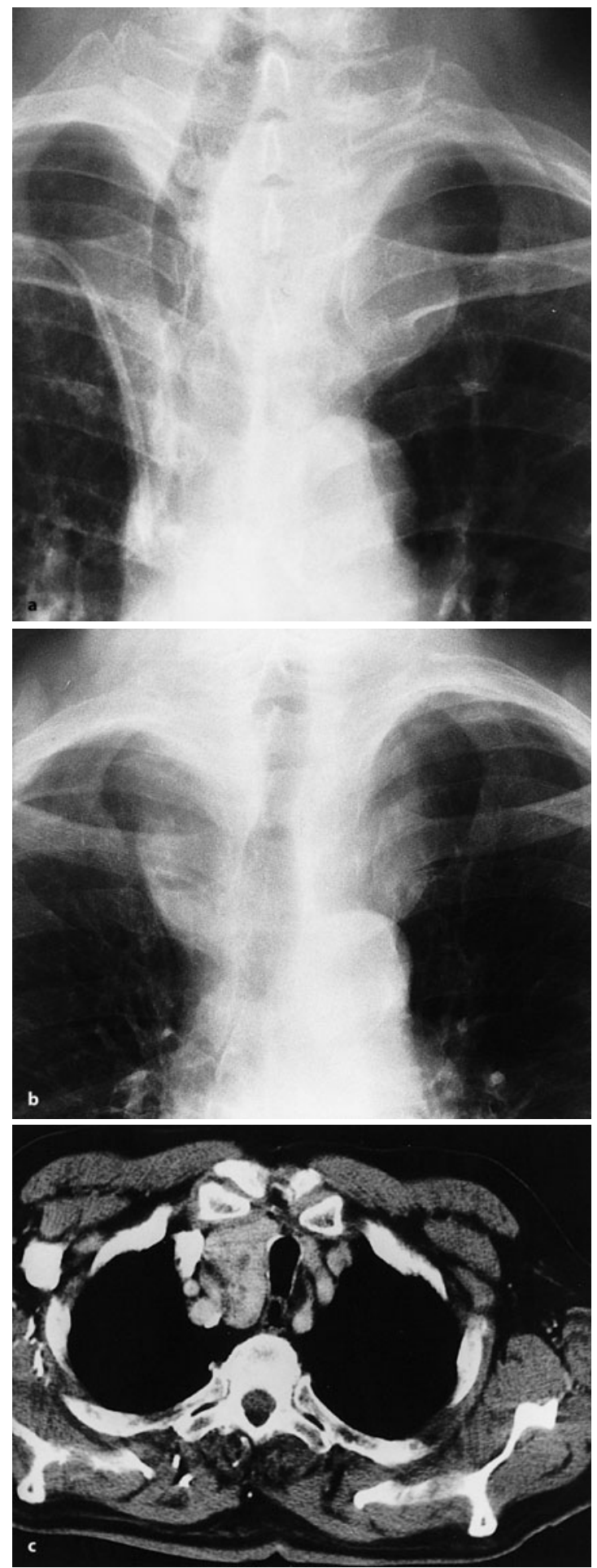

Abb. 17.17 a-c. Retrosternale Struma nodosa bei drei Patienten. Sagittale Thoraxübersichtsaufnahme mit a Nachweis einer links betonten Raumforderung im oberen Mediastinum, die zu einer Trachealverlagerung nach rechts geführt hat, und $\mathbf{b}$ einer Raumforderung im oberen Mediastinum, die die Trachea von beiden Seiten einengt. c CT nach intravenöser Kontrastmittelgabe mit Nachweis eines nach retrosternal reichenden rechten Schilddrüsenlappens, dessen Parenchym durch hypodense Adenome durchsetzt ist und der die Trachea einengt

Gelegentlich kann der Thymus im Erwachsenenalter als kleine, noduläre, fettdurchsetzte retrosternale Struktur persistieren. Bei Kindern und Jugendlichen existieren keine verlässlichen Messwerte zur Unterscheidung eines normalen Thymus von einer Thymushyperplasie. Nach dem 20. Lebensjahr ist bei einem maximalen Querdurchmesser über $15 \mathrm{~mm}$ von einer Thymushyperplasie auszugehen. Hyperplastische Veränderungen als Folge einer schweren Allgemeinerkrankung oder einer Chemotherapie können sich im Verlauf von Monaten zurückbilden. In etwa $25 \%$ der Fälle bleibt jedoch eine Organvergrößerung von $>50 \%$ des Ausgangswertes bestehen.

Thymome finden ihre schnittbilddiagnostische Entsprechung in rundlich-ovalären, exzentrisch im vorderen oberen Mediastinum gelegenen überwiegend soliden Raumforderungen (Abb. 17.19). Lobulierte Randkonturen weisen auf kleinere intraparenchymatöse Raumforderungen hin. Zysten und Verkalkungen kommen unabhängig von der Dignität bei etwa $25 \%$ der Thymome vor. Verkalkungen können diffus in der Tumormatrix verteilt oder in der Tumorperipherie lokalisiert sein. Computertomographisch gelingt nach intravenöser Kontrastmittelgabe eine im Vergleich zur Nativdiagnostik bessere Demarkierung intratumoraler Zysten. MR-tomographisch zeichnen sich Thymome durch eine niedrige, der Muskulatur annähernd isointense Signalintensität in T1-Wichtung und eine hohe Signalintensität in T2-Wichtung aus. Im Gegensatz zu mediastinalem Fettgewebe ist die Signalintensität von Thymomen in T2-Wichtung in der Regel inhomogen. 30-40\% der Thymome wachsen lokal infiltrierend. Verbreiterungen der Pleura und des Perikards, Pleura- und Perikardergüsse, eine Obliteration der Fettlinien der Thoraxwand und Arrosionen des Sternums zeigen ein infiltratives Wachstum an. Da die Tumorkapsel mit dem Perikard und der Pleura verwachsen sein kann, deutet ein Fehlen der entsprechenden Fettlinien nicht zwangsläufig auf eine Infiltration hin. Eine sichere Dignitätszuordnung von Thymomen ist weder computer- noch MR-tomographisch möglich, sodass Thymome nach wie vor operativ exploriert werden müssen (Abb. $17.18 \mathrm{c}, \mathrm{d})$. 

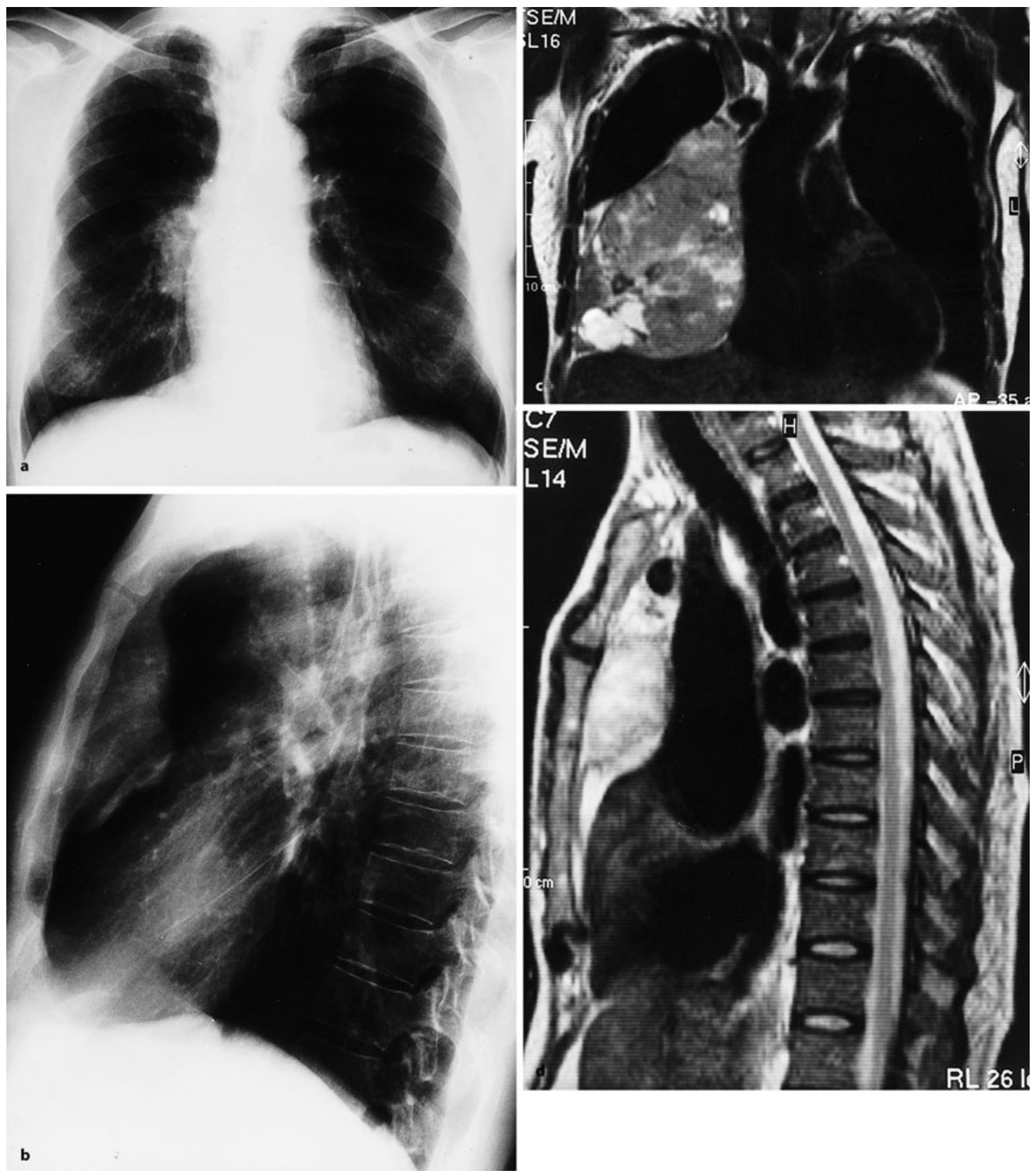

Abb. 17.18a-d. Thymuskarzinom. Thoraxübersichtsaufnahme im sagittalen $\mathbf{a}$ und im seitlichen Strahlengang $\mathbf{b}$ mit Nachweis einer retrosternalen, glatt begrenzten Verdichtung.

Das T2-gewichtete koronale c und sagittale d Turbo-SpinEcho-Bild eines anderen Patienten zeigt eine inhomogen strukturierte Tumormatrix 


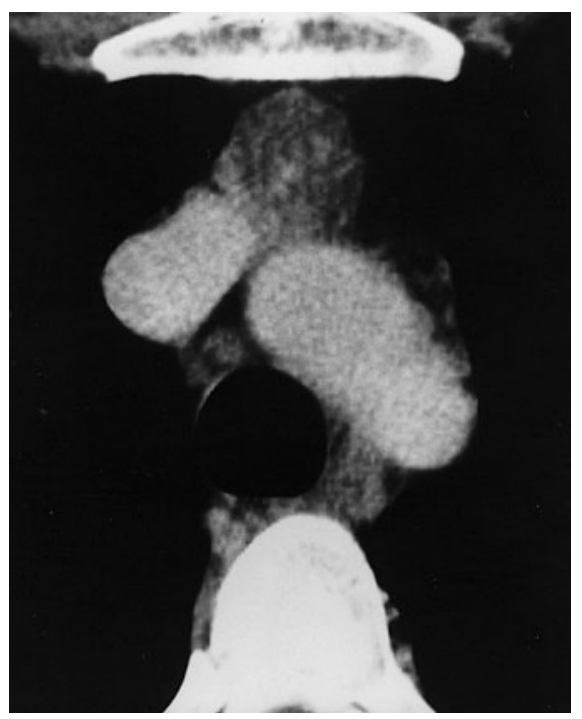

Abb. 17.19. Thymom. CT nach intravenöser Kontrastmittelgabe

Thymolipome können sehr groß werden. Sie passen sich aufgrund ihrer weichen Konsistenz den mediastinalen Strukturen an, ohne sie zu verdrängen. Sie können in wechselndem Ausmaß bindegewebige Anteile aufweisen, sodass ihre computertomographische Dichte gegenüber reinem Fettgewebe erhöht ist. MR-tomographisch ist eine im Vergleich zu Fettgewebe erhöhte Signalintensität sowohl in T1-als auch in T2-Wichtung charakteristisch. Einblutungen in Thymuszysten sind häufig, sodass der Zysteninhalt je nach Proteingehalt Dichtewerte zwischen -10 und +80 H.E. aufweisen kann. MR-tomographisch ist eine mit zunehmender T2-Wichtung ansteigende Signalintensität des Zysteninhalts diagnoseweisend. Wandverkalkungen werden als Ausdruck stattgehabter Einblutungen gewertet.

Keimzelltumoren sind häufig im vorderen oberen Mediastinum und hier insbesondere im Thymuslager lokalisiert. Teratome weisen entsprechend ihrer Abstammung aus allen drei Keimblattanlagen inhomogene, von fett- über wasser- bis zu kalziumäquivalente Dichten bzw. Signalintensitäten auf (Abb. 17.16c). Zwischen Dermoidzysten und zystischen Teratomen bestehen histologisch wie bildmorphologisch fließende Übergänge. Eine unscharfe Begrenzung, zentrale Nekrosen und eine Kompression oder Verlagerung der Nachbarstrukturen kennzeichnen ein malignes Teratom. Dermoidzysten bestehen typischerweise aus einer oder mehreren Zysten mit glatt konturierten, oft verkalkten Wänden. Sie reichen gelegentlich in das mittlere Mediastinum oder nach pulmonal. Der Zysteninhalt hat computer- und MRtomographisch eine wasser- oder fettäquivalente Bild- morphologie. Als pathognomonisch gilt der Nachweis von Zähnen, Haaren, Knochenanlagen und Fett-Flüssigkeits-Spiegeln. Bei Seminomen, Chorionkarzinomen, Embryonalzellkarzinomen und ihren Mischformen handelt es sich um solide, eher lobulierte Tumoren. Seminome zeichnen sich durch ihre homogene Gewebedichte aus. Zystische Veränderungen werden in weniger als einem Viertel aller Seminome, Verkalkungen nur gelegentlich beobachtet. Die übrigen malignen Keimzelltumoren dagegen zeigen aufgrund von Nekrosen und Einblutungen ein inhomogenes Tumorstroma. Verkalkungen sind selten, Fetteinschlüsse ungewöhnlich. Schnelles Tumorwachstum, lokale Infiltrationszeichen und der Nachweis von Fernmetastasen beweisen die Malignität. In bis zu $40 \%$ der Fälle finden sich Verdrängungen oder Kompressionen der zentralen Atemwege und der V. cava superior. Bei extragonadalen malignen Keimzelltumoren muss der Primärcharakter der mediastinalen Läsion durch Ausschluss gonadaler und retroperitonealer Tumoren gesichert werden. Bei soliden Resttumoren nach Radiochemotherapie kann ohne langfristige Verlaufskontrollen bildmorphologisch nicht zwischen residualem Narbengewebe, Resttumorgewebe und der Ausdifferenzierung eines malignen Teratoms differenziert werden.

Lymphangiome und Hämangiome finden ihre computer- bzw. MR-tomographische Entsprechung in glatt begrenzten, dünnwandigen Raumforderungen von wasseräquivalenter Dichte bzw. Signalintensität bei Nativuntersuchungen (Parker 1997). Ein hoher Fettgehalt der Lymphe spiegelt sich in einer Signalintensitätsanhebung in T1-Wichtung wieder. Hämangiome zeigen im Gegensatz zu Lymphangiomen nach intravenöser Kontrastmittelgabe ein starkes Enhancement. Phlebolithen innerhalb eines Hämangioms sind computertomographisch aufgrund der Kalkdichte diagnostizierbar.

Unter einer intrathorakalen bzw. retrosternalen Struma wird die Fortsetzung des Gewebes einer orthotopen Schilddrüse nach intrathorakal verstanden. Die transkutane Sonographie ermöglicht über ein juguläres oder parasternales Schallfenster die Diagnose der intrathorakalen Ausdehnung. Solide (Adenome, Malignome), liquide (benigne Zysten, nekrotisch zerfallende Malignome) und regressiv verkalkte Parenchymläsionen sind durch ihre Echogenitätsabweichungen vom gesunden Schilddrüsengewebe in den einsehbaren Parenchymanteilen sicher zu diagnostizieren. Durch knöcherne (Rippen, Sternum) oder lufthaltige Strukturen (Trachea, Lunge) überlagerte Drüsenanteile sind der transkutanen Sonographie jedoch nicht zugänglich.

Computertomographisch lassen sich Verlagerungen und Kompressionen der Trachea und der brachiozephalen Gefäße durch eine retrosternale Struma 

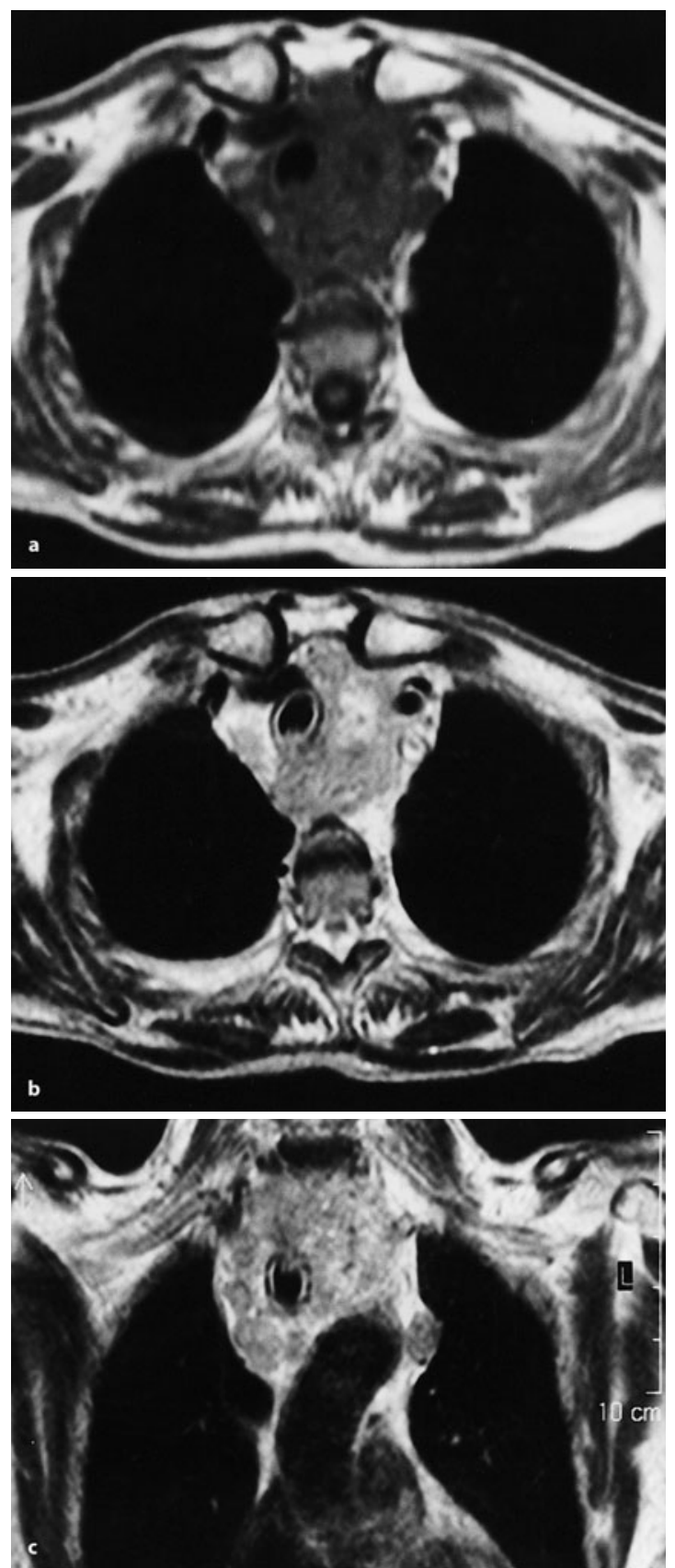

Abb. 17.20 a - c. Schilddrüsenkarzinom. T1-gewichtetes transversales Spin-Echo-Bild vor intravenöser Kontrastmittelgabe (a) und transversales (b) und koronales (c) T1-gewichtetes Spin-Echo-Bild nach intravenöser Kontrastmittelgabe zuverlässlich nachweisen (Abb. 17.17c). Regressive Verkalkungen und Zysten sind anhand ihrer Dichtewerte eindeutig zuzuordnen. Lässt sich eine strangförmige Verbindung zwischen einem Mediastinaltumor und der Schilddrüse nachweisen, so ist die Diagnose einer Struma endothoracica wahrscheinlich. Eine vergleichsweise hohe native Gewebedichte (ca. 70 H.E.) und ein starkes Enhancement nach transvenöser Gabe eines jodhaltigen Kontrastmittels sind weitere Hinweise auf das Vorliegen von Schilddrüsengewebe. Wegen der hohen Dichte des gesunden Drüsenparenchyms stellen sich fokale Parenchymläsionen sowohl im Nativ- als auch im Kontrastscan als hypodense intraparenchymatöse Raumforderungen da. Zysten weisen nach intravenöser Kontrastmittelgabe wasseräquivalente Dichtewerte ohne Dichteanstieg auf. Strumagewebe stellt sich MRtomographisch signalinhomogen dar (Cyna-Gorse 1996). Größere Verkalkungen führen zu Signalintensitätsminderungen in allen Wichtungen. Unkomplizierte Zysten sind aufgrund ihrer wasseräquivalenten Signalintensitäten eindeutig zuzuordnen. Einblutungen zeigen je nach Alter variierende Signalintensitäten. Eine Dignitätszuordnung solider thyreoidaler Raumforderungen ist mit allen bildgebenden Verfahren nur eingeschränkt möglich (Abb.17.20). Eine glatte Berandung und regressive Verkalkungen machen ein benignes Adenom, ein Übergreifen auf Nachbarstrukturen und Lymphknotenvergrößerungen ein Karzinom wahrscheinlich. Bei entsprechendem klinisch-szintigraphischen Verdacht ist die Diagnose jedoch histologisch zu klären (Freitas 1994).

Bei voroperierten Patienten wird die Sensitivität im Nachweis vergrößerter Nebenschilddrüsen im Methodenvergleich mit 47-82\% für die Sonographie, mit $44-66 \%$ für die Computertomographie und mit $50-75 \%$ für die MR-Tomographie angegeben (Higgins 1993; Saleeb 1999). Vergrößerte Nebenschilddrüsen stellen sich sonographisch als rundlichovaläre, glatt berandete, echoarme Raumforderungen dar, die unter Bevorzugung der rechten Seite meistens dorsokaudal der unteren Schilddrüsenpole gelegen sind. Bei der Frage nach ektopen Nebenschilddrüsenadenomen ist besonders auf den tracheoösophagealen Winkel und das Thymuslager zu achten. Wegen der geringen Größe ist in CT und MRT eine Untersuchungsschichtdicke $£ 5 \mathrm{~mm}$ zu empfehlen. MR-tomographische Untersuchungen sollten zur Verbesserung des Signal-zu-Rausch-Verhältnisses mit einer Oberflächenspule erfolgen.

Computertomographisch haben Nebenschilddrüsenadenome eine muskeläquivalente Dichte. Parenchymhypodensitäten sprechen für Einblutungen oder Nekrosen. Parenchymverkalkungen und -zysten werden nur selten gefunden. Die Mehrzahl der Ade- 
nome zeigt im Gegensatz zu mediastinalen Lymphknoten ein homogenes, starkes Kontrastmittelenhancement.

Die MRT ist die Methode der Wahl zur Suche nach ektopen Nebenschilddrüsen. Zur Unterscheidung von ektopem Nebenschilddrüsen- und mediastinalem Fettgewebe sind fettunterdrückte T1- und T2-gewichtete Sequenzen sowohl vor als auch nach intravenöser Kontrastmittelgabe hilfreich. Nebenschilddrüsenadenome stellen sich in T1-Wichtung mehrheitlich signalarm bis intermediär und in T2-Wichtung signalreich dar (Freitas 1994; Higgins 1993; Soler 1996). Bei einer TE-Zeit über $60 \mathrm{~ms}$ ist die Signalintensität von vergrößerten Nebenschilddrüsen in der Regel höher als die von Fett. Gelegentlich werden jedoch auch in T1- und T2-Wichtung signalarme Adenome beobachtet, die sich histologisch durch ältere Einblutungen und Fibrosierungen auszeichnen. Subakute Einblutungen können zu einer hohen Signalintensität in T1- und T2-Wichtung führen. Analog den Verhältnissen bei der Computertomographie kommt es nach intravenöser Kontrastmittelgabe zu einem starken Enhancement, das bei T1-gewichteten, nicht fettunterdrückten Sequenzen $\mathrm{zu}$ einer Angleichung der Signalintensität der $\mathrm{Ne}$ benschilddrüse an die des umgebenden Fettgewebes führt. Die MR-tomographische Bildmorphologie vergrößerter Nebenschilddrüsen weist Ähnlichkeiten zur Abbildung von Lymphknoten und Nervenstrukturen auf, sodass sich Verwechslungsmöglichkeiten ergeben.

\section{Mittleres Mediastinum}

\section{Pathologie und Klinik}

Primärtumoren des mittleren Mediastinums sind selten (Tabellen 17.1, 17.3; Anayanwu u. Krysa 1991; Anayanwu 1991; Chang 1994; Daldrup 1998; Hofmann 1991; Kohman 1993; Lange 1996; Merten 1991; Schaefer-Prokop 1998; Siegel 1996; Strolle 1997; Wegener 1992). Trachealtumoren sind eine Rarität. Histologisch kommen Plattenepithelkarzinome, adenoidzystische Karzinome (Zylindrome), Neurofibrome, Papillome und mesenchymale Tumoren in Betracht. Polypöse Tumoren sind meist benigne. Malignome breiten sich in der Regel langstreckig infiltrierend entlang der Trachealwand aus und sind irregulär begrenzt. Trachealtumoren verursachen erst dann Symptome, wenn das Lumen auf $\geqq 75 \%$ des Ausgangsdurchmessers eingeengt ist und eine Infiltration der paratrachealen Strukturen vorliegt. Als klinische Zeichen sind Husten, Hämoptysen, Dyspnoe und Stridor zu nennen. Die Differenzierung eines Trachealkarzinoms von einem metastasierenden Bronchialkarzinom ist nur im frühen Tumorstadium durch Analyse der topographischen Pathoanatomie mög- lich. Auch die Abgrenzung eines infiltrierend wachsenden Trachealkarzinoms von einem Ösophaguskarzinom kann mit bildgebenden Verfahren Schwierigkeiten bereiten. Durch nekrotischen Zerfall eines infiltrierend wachsenden Tracheal- oder Ösophaguskarzinoms können Fisteln zwischen beiden Hohlraumstrukturen und dem Mediastinum entstehen.

Paragangliome oder Chemodektome gehören $\mathrm{zu}$ den neurogenen katecholaminproduzierenden Tumoren und sind in etwa $10 \%$ der Fälle maligne. Sie gehen von den zwischen A. pulmonalis und der Aorta lokalisierten paraganglischen Zellen der Endplatten des N. vagus aus.

\section{Projektionsradiographie}

Auf der Thoraxübersichtsaufnahme führen Raumforderungen des mittleren Mediastinums bei entsprechender Größe zu einer Mediastinalverbreiterung und Mediastinalverdichtung (Abb. 17.21, 17.22; Allen 1983; Chang 1994; Kohman 1993; Lackner 1998; Lange 1996; Merten 1991; Murray 1992; O’Donovan 1994). Trachealverlagerung und Trachealkompressionen sind häufig auf eine Struma zurückzuführen. Trachealtumoren stellen sich projektionsradiographisch bei entsprechender Größe als polypoide oder breitflächige intraluminale Verdichtungen dar. Eine Verbreiterung der physiologischen Verdichtung zwischen Trachea und Ösophagus im Seitbild über $4 \mathrm{~mm}$ deutet auf Fremdgewebe hin. Hinweise auf das Vorliegen eines Ösophaguskarzinoms können eine Verbreiterung der retrotrachealen Linie (Seitbild) über $4 \mathrm{~mm}$, eine Verlagerung der azygoösophagealen Linie (Sagittalbild) und eine Obliteration des aortopulmonalen Fensters (Sagittalbild) geben. Eine Vergrößerung des Bifurkationswinkels über $70^{\circ}$ und eine Verlagerung des distalen Ösophagus machen eine Raumforderung im unteren mittleren Mediastinum wahrscheinlich. Durch eine durchleuchtungsgesteuerte Kontrastmitteldarstellung des Ösophagus lässt sich überprüfen, ob die entsprechende Mediastinalveränderung vom Ösophagus ausgeht, diesen nur verlagert oder infiltriert (Abb.17.23, 17.24b). Auf gleichem Wege lassen sich ösophagotracheale und ösophagomediastinale Fisteln nachweisen.

\section{Schnittbilddiagnostik}

Gutartige Trachealtumoren sind in der Regel glatt begrenzte, intraluminale weichteildichte (CT) bzw. intermediär intense (MRT) Raumforderungen unter $2 \mathrm{~cm}$ Durchmesser. Bösartige Trachealtumoren weisen ein längerstreckiges, exophytisches Wachstum auf. Die Beurteilung einer Infiltration der Nachbarstrukturen ist computer- und MR-tomographisch meist unsicher (Bittner 1998; Chang 1994; Daldrup 1998; Ferguson 1998; Kohman 1993; Lange 1996; Lesko 1999; Merten 1991; Murray 1992; Nwose 1998; 

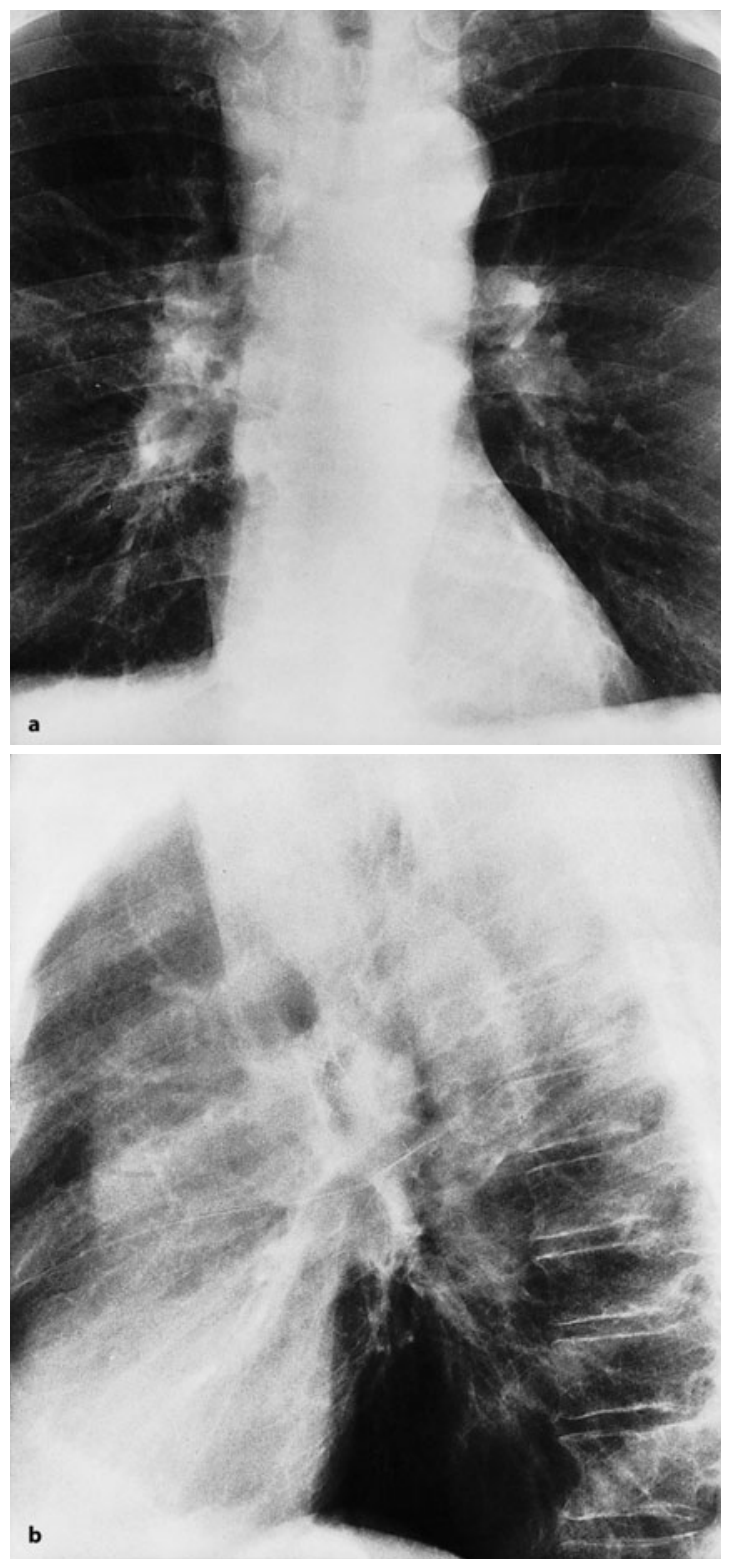

Abb. 17.21 a, b. Primärtumor des mittleren Mediastinums. Histologisch handelt es sich um ein Ösophaguskarzinom. a Thoraxübersichtsaufnahme im sagittalen und $\mathbf{b}$ im seitlichen Strahlengang

Schaefer-Prokop 1998; Siegel 1996; Wegener 1992; Wilson 1994). Während bei ausgedehnten Umgebungsinfiltrationen eindeutige Befunde zu erheben sind, bereitet der Ausschluss einer beginnenden Infiltration methodische Schwierigkeiten. Eine sichere Dignitätszuordnung ist daher oft nicht möglich, sodass eine bioptische Klärung anzustreben ist.

Ösophagustumoren stellen sich als exzentrische oder konzentrische Wandverbreiterungen der Speise-

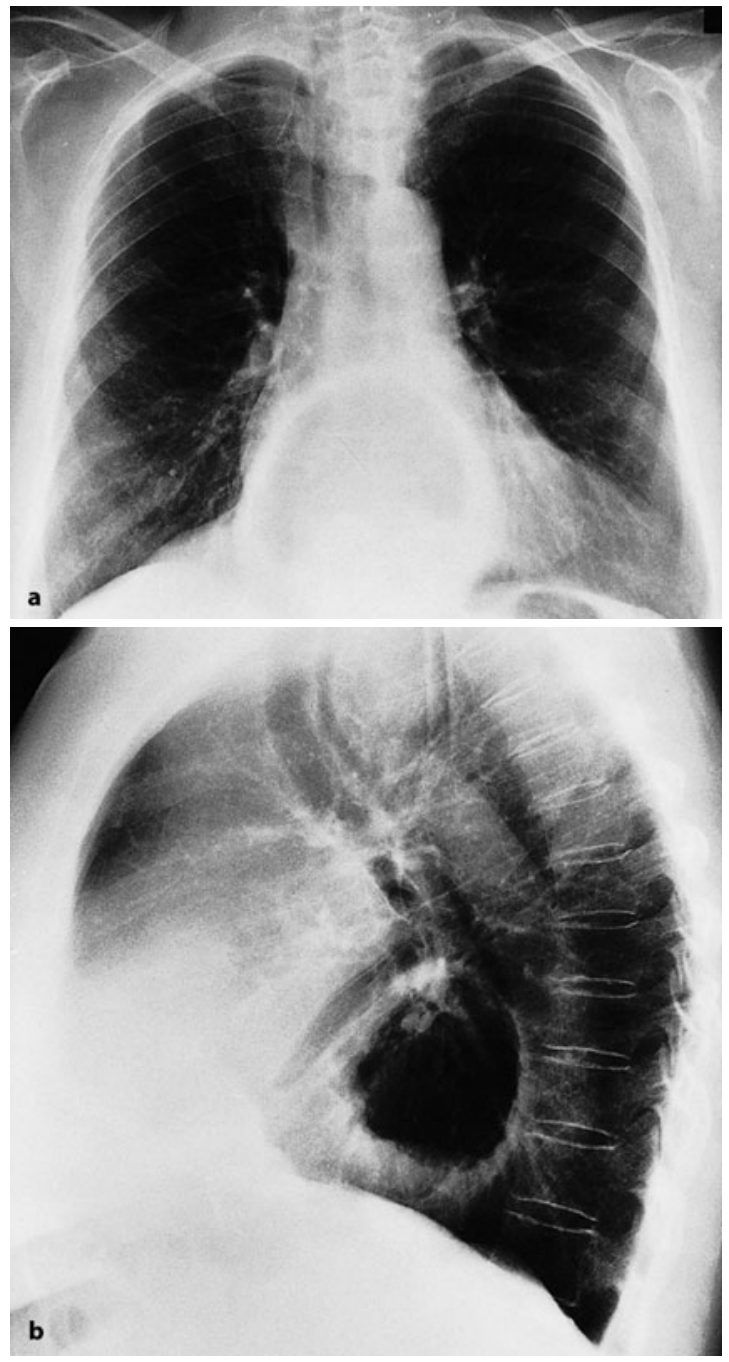

Abb. 17.22a, b. Axiale Hernie. Thoraxübersichtsaufnahme im sagittalen a und seitlichen Strahlengang $\mathbf{b}$ mit Nachweis einer glatt begrenzten retrokardialen Raumforderung mit Luft-Flüssigkeits-Spiegel

röhre dar, die im Falle eines Karzinoms bevorzugt an den drei physiologischen Ösophagusengen lokalisiert sind (Abb. 17.24). Der Verlust der peritrachealen bzw. periösophagealen Fettschicht ist bei beiden Tumorentitäten Kriterium eines frühen wandüberschreitenden Wachstums. Als sichere Zeichen eines organüberschreitenden Tumorwachstums gelten eine Verlagerung und Kompression der Speiseröhre bzw. der Luftröhre, tracheale bzw. ösophageale Fisteln, die an einem unphysiologischen Übertritt von peroral appliziertem Kontrastmittel in Luftwege, Mediastinum und Pleuraraum erkennbar sind, und Wirbelkörperarrosionen. 

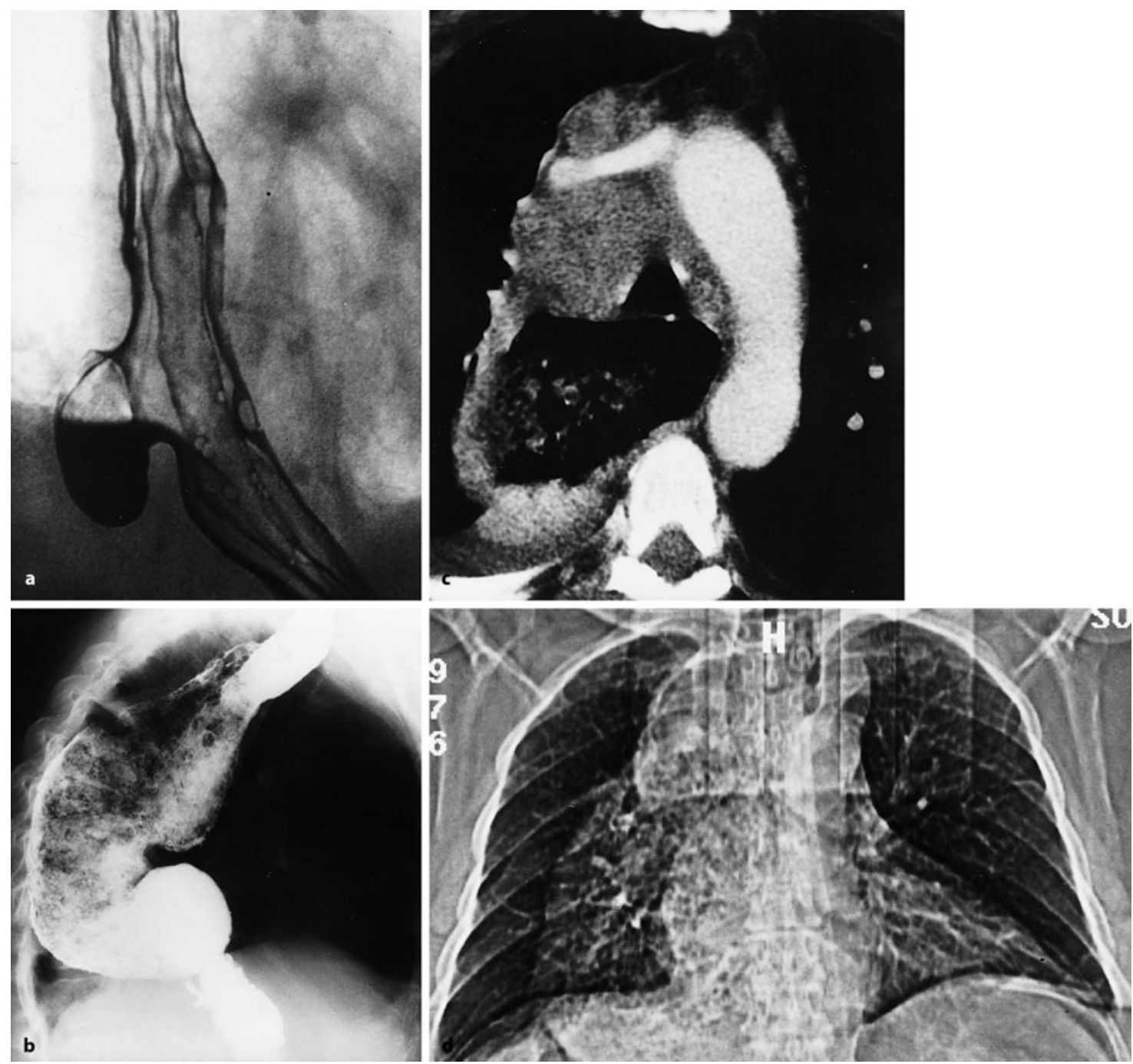

Abb. 17.23 a-d. Kontrastmittelgestützte Ösophagusdiagnostik. a Epiphrenisches Divertikel: Durchleuchtungsgesteuerte Zielaufnahme. b-d Achalasie: Durchleuchtungsgesteuerte Zielaufnahme (b), CT-Topogramm (c) und CT nach intravenöser

Kontrastmittelgabe (Ausschnittsvergrößerung) (d). Zusätzlich weichteildichte entzündliche Lymphknotenvergrößerungen im vorderen und mittleren oberen Mediastinum infolge einer Tuberkulose (c, d)

Paragangliome sind überwiegend im aortopulmonalen Fenster, seltener im hinteren Mediastinum lokalisiert. Es handelt sich um weichteildichte Raumforderungen, die aufgrund ihrer starken Vaskularisation nach intravenöser Kontrastmittelgabe ein ausgeprägtes Enhancement aufweisen.

\section{Hinteres Mediastinum}

\section{Pathologie}

Neurogene Tumoren stellen bei Erwachsenen etwa $20 \%$ und bei Kindern etwa 35\% der mediastinalen Tumoren (Tabelle 17.1 - 17.3, 17.6; Anayanwu u. Krysa
1991; Anayanwu 1991; Chang 1994; Daldrup 1998; Hofmann 1991; Kawashima, Kohman 1993; Lange 1996; Merten 1991; Nwose 1998; Parker 1997; SchaeferProkop 1998; Schulman 1998; Siegel 1996; Strolle 1997; Taki 1996; Wegener 1992).

Mit Ausnahme des Paraganglioms (Chemodektoms) sind sie im hinteren Mediastinum gelegen (90\% der neurogenen Mediastinaltumoren) und repräsentieren in dieser Lokalisation die häufigsten Primärtumoren (75\% der Tumoren des hinteren Mediastinums). Neurogene Tumoren stammen von den Nervenscheiden, den Nervenzellen oder dem perineuralen Bindegewebe ab. Tumoren der Nerven- 

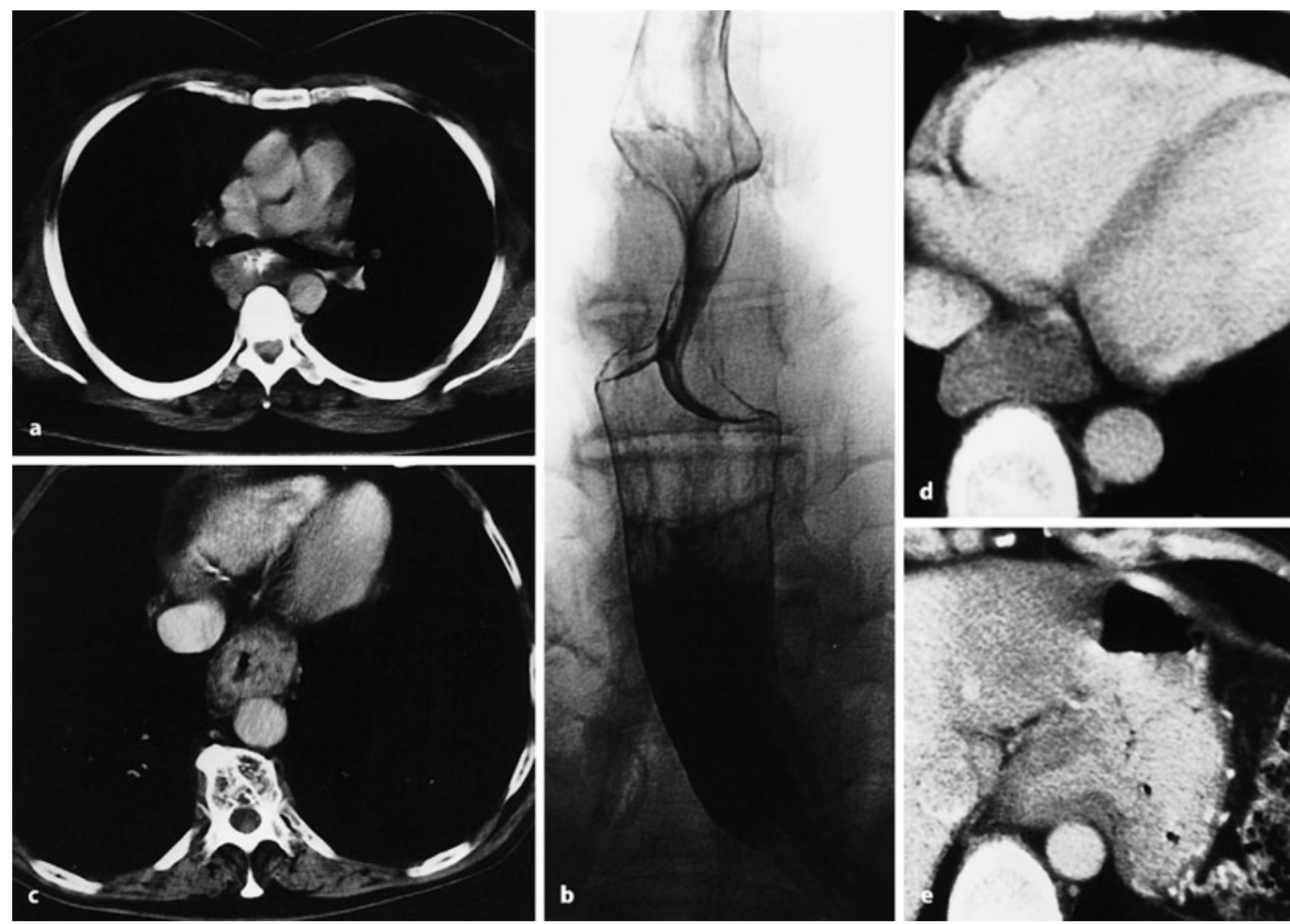

Abb. 17.24a-e. Computertomographische Darstellung von Ösophagustumoren. a, b Leiomyom: Subkarinärer Ösophagustumor, der zu einer Passageverzögerung des peroral gegebenen Kontrastmittels führt (a) und im Ösophagusbrei-

schluck glatte Wandkonturen aufweist (b). c Tumor im mittleren Speiseröhrendrittel mit wandüberschreitendem Wachstum. Als Nebenbefund Koronarsklerose. d, e Auf die Kardia übergreifendes Ösophaguskarzinom

scheide überwiegen zahlenmäßig und sind praktisch immer gutartig. Von den Nervenzellen und dem perinervalen Bindegewebe ausgehende Tumoren sind eher bösartig. Je nach histologischem Ursprung werden drei Tumortypen differenziert:

- Von peripheren Nerven ausgehende Neurinome, Neurofibrome und Neurilemome. Neurofibrome stammen von dem endoneuralen Gewebe ab, Neurilemome (Schwannome) von der SchwannScheide.

- Von den sympathischen Nervenzellen in Ganglien ausgehende Ganglioneurome (benigne), Ganglioneuroblastome (niedrigmaligne) und Neuroblastome (hochmaligne, Abb. 17.25).

- Von den paraganglischen Zellen abstammende Phäochromozytome.

Laterale Meningozelen und Meningomyelozelen sind seltene Anomalien des Spinalkanals, die aus einer uni- oder bilateralen Hernierung der Leptomeninx durch ein Neuroforamen entstehen. Meningozelen enthalten ausschließlich Liquor, Meningomyelozelen zusätzlich Nervengewebe. Meningozelen treten in der Regel solitär auf und werden im gesamten hinteren
Mediastinum von der oberen Thoraxapertur bis zum Zwerchfell beobachtet. Als Begleitbefund findet sich häufig eine Kyphoskoliose, wobei die Meningozele üblicherweise konvexseitig am Scheitelpunkt der Wirbelsäulenverbiegung liegt.

Die mesenchymalen Tumoren des hinteren Mediastinum sind mehrheitlich maligne. Die extrem seltenen Lipofibrosarkome führen typischerweise zu Verdrängungen und Infiltrationen der Nachbarorgane.

Paraspinale Weichteilprozesse können vertebragenen Ursprungs sein. Die Röntgen-, CT- und MRMorphologie der differentialdiagnostisch in Betracht

Abb. 17.25 a-g. Primärtumor des hinteren Mediastinums. Histologisch handelt es sich um Neuroblastome. a Die sagittale und $\mathbf{b}$ die seitliche Thoraxübersichtsaufnahme zeigen eine glatt begrenzte Raumforderung im hinteren Mediastinum (Pfeil). c Sagittale und d seitliche Thoraxübersichtsaufnahme eines anderen Patienten mit einer vom hinteren ins mittlere Mediastinum übergreifenden Raumforderung, e natives CT, $\mathbf{f}$ transversales und $\mathbf{g}$ koronales, T2- gewichtetes Turbo-Spin-Echo-Bild 

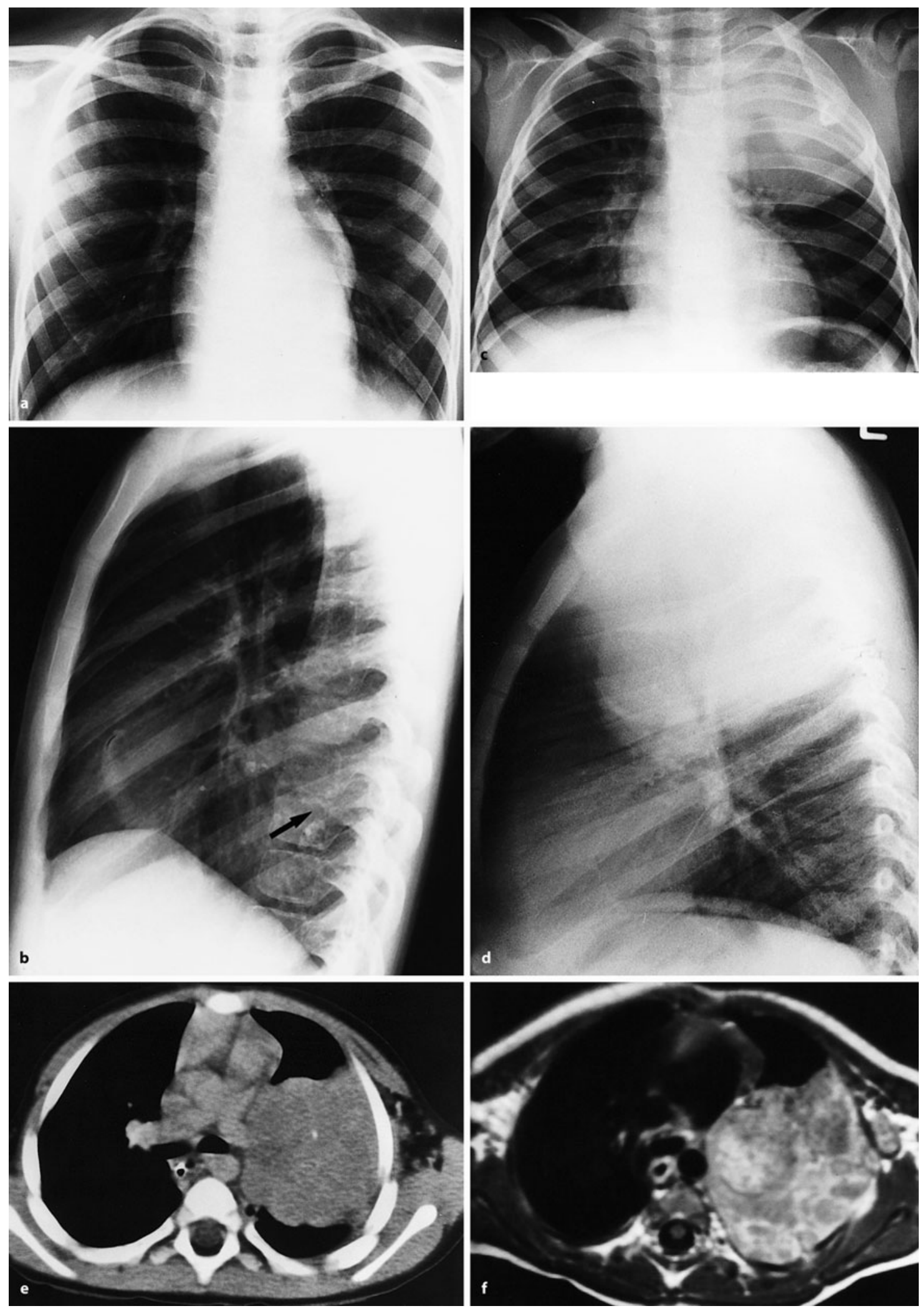


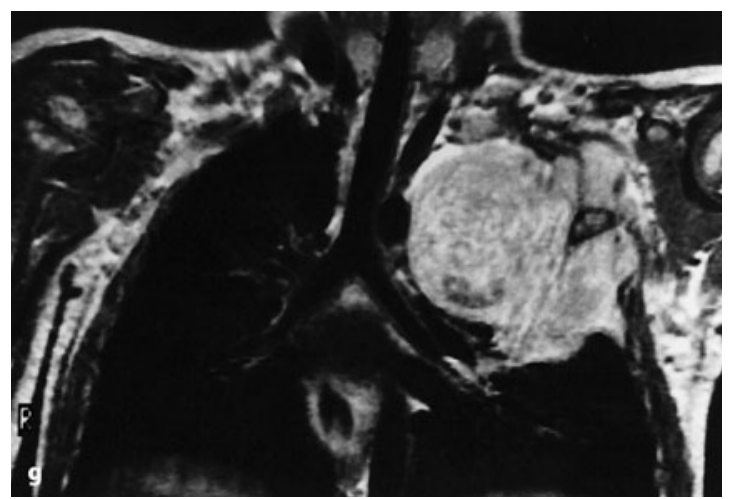

Abb. 17.25 g. Legende s. S. 30

zu ziehenden Erkrankungen - Spondylitis/Spondylodiscitis (Abb.17.26), primärer und sekundärer Knochentumor und Traumafolgen - sind in Band „Kopf - Hals“ dieser Reihe besprochen.

\section{Klinik}

Neurogene Tumoren und laterale Meningozelen/ Meningomyelozelen sind in der Mehrzahl asymptomatisch und werden als Zufallsbefund auf der Thoraxübersichtsaufnahme entdeckt (Chang 1994; Daldrup 1998; Kawashima, Kohman 1993; Lange 1996; Merten 1991; Schaefer-Prokop 1998; Schulman 1998; Siegel 1996; Strolle 1997; Taki 1996; Wegener 1992). Symptome lassen sich - wenn vorhanden - auf die raumfordernde Wirkung des Tumors, ein intraspinales Tumorwachstum und/oder eine Infiltration von Umgebungsstrukturen zurückführen (Abb. 17.27).

Neurinome, Neurofibrome und Schwannome sind Tumoren junger Erwachsener und häufig mit Neurofibromen in anderen Körperabschnitten vergesellschaftet (M. von Recklinghausen). Nach Resektion solitärer Tumoren besteht keine Rezidivneigung. Mediastinale Neurofibrome, die mit einer Neurofibromatose vergesellschaftet sind, weisen eine Entartungsneigung auf.

Ganglioneurome betreffen meist ältere Kinder bis junge Erwachsene, Ganglioneuroblastome Kinder unter 10 Jahren. Neuroblastome sind die häufigsten extrakraniellen soliden Tumoren im Kindesalter. In $60 \%$ der Fälle sind Kinder unter 2 Jahren und in bis zu $90 \%$ Kinder unter 5 Jahren betroffen. Selten sind Neuroblastome angeboren. In 15\% aller Neuroblastomerkrankungen findet sich ein Mediastinalbefall. Zwei Drittel aller an einem Neuroblastom erkrankten Kinder werden aufgrund hämatogener Metastasen symptomatisch. Neuroblastome, weniger häufig Ganglioneuroblastome und Ganglioneurome können metabolisch aktiv sein. Dies ist an erhöhten Spiegeln



Abb. 17.26 a, b. Spondylodiscitis. a Ausschnittsvergrößerung einer sagittalen Thoraxübersichtsaufnahme, b CT nach intravenöser Kontrastmittelgabe (Ausschnittsvergrößerung). Entzündliche Destruktion der bandscheibennahen Kortikalis und entzündlicher paravertebraler Weichteilprozess

von Katecholaminen im Blutserum und Katecholaminabbauprodukten (Homovanillinmandelsäure) im Urin erkennbar. Vasoaktives intestinales Peptid (VIP) kann einen Bluthochdruck, eine Flush-Symptomatik und wässrige Diarrhöen verursachen. Die einen Epinephrinvorläufer nutzende und daher für Neoplasmen sympathischen Ursprungs relativ spezifische Jod-123 MIBG(Meta-Iodo-Benzyl-Guanidin)Szintigraphie eignet sich zur Primärdiagnostik und zum Metastasenscreening beim Neuroblastom. Ungünstige Prognosefaktoren sind ein höheres Lebens- 




alter zum Zeitpunkt der Diagnose, ein großes Tumorvolumen, ein undifferenzierter Zelltyp, ein Tumorstadium $\geqq$ III und eine extrathorakale Lokalisation des Primärtumors. Eine intraspinale Infiltration verschlechtert die Prognose nicht.

Bei Erwachsenen lassen hypertone Krisen, die mit einer Flush-Symptomatik einhergehen, in Verbindung mit einer mediastinalen Raumforderung an das sehr seltene mediastinale Phäochromozytom denken.

Laterale Meningozelen/Meningomyelozelen führen aufgrund ihrer langsamen Größenzunahme wie Neurinome oder Neurofibrome zu einer druckbedingten Verbreiterung des betroffenen Neuroforamens. Meningozelen und Meningomyelozelen werden meistens als Zufallsbefund in der 4. und 5. Lebensdekade diagnostiziert. Ebenso wie die im hinteren Mediastinum gelegenen Neurofibrome und Neurofibrosarkome sind sie in etwa $75 \%$ der Fälle mit einer Neurofibromatose vergesellschaftet.

\section{Projektionsradiographie}

Auf der sagittalen Thoraxübersichtsaufnahme sind Raumforderungen im hinteren Mediastinum aufgrund des unterschiedlichen Objekt-Film-Abstandes (Silhouetten-Zeichen) gut von dem Herzrand abzugrenzen (Abb. 17.25 a; Chang 1994; Kohman 1993; Lackner 1998; Lange 1996; Merten 1991; Murray 1992; Schulman1998; Taki 1996). Bei unmittelbarem Kontakt verliert die Kontur des dorsalen Aorten-

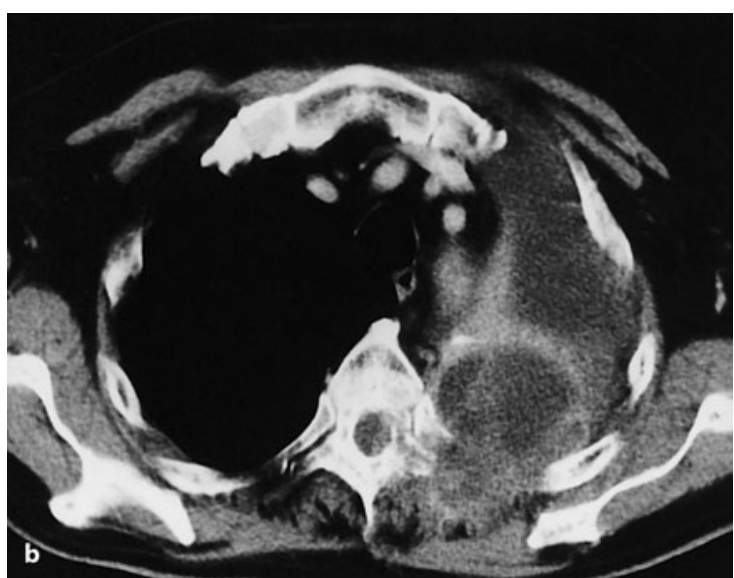

Abb. 17.27 a, b. Computertomographische Darstellung von Pancoast-Tumoren. Zwei Patienten mit peripheren Bronchialkarzinomen im linken Oberlappen, Infiltration des hinteren Mediastinums und beginnender Destruktion eines Wirbelkörpers (Ausschnittsvergrößerung) (a) bzw. Destruktion einer dorsalen Rippe (b)

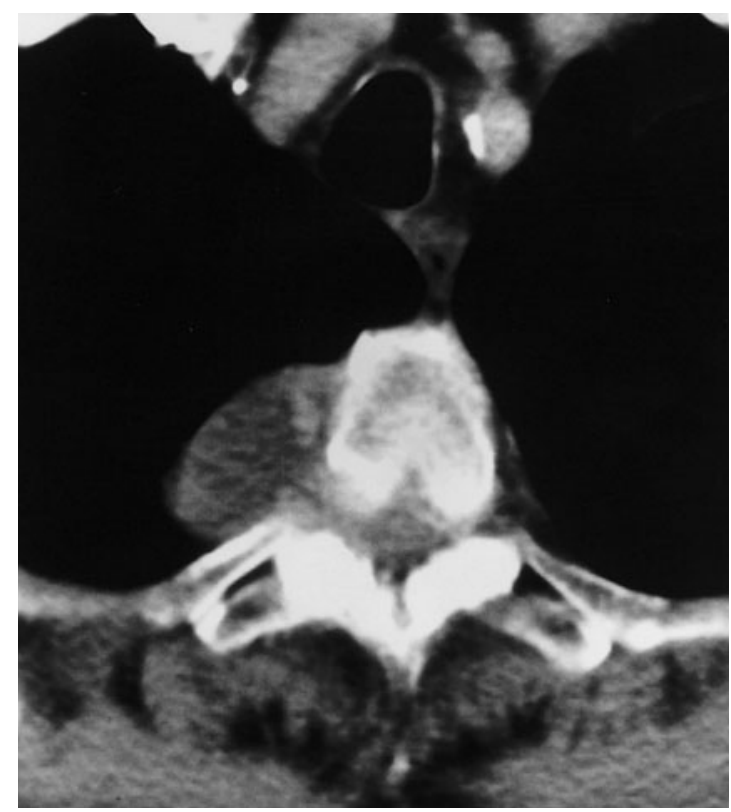

Abb. 17.28. Neurinom. CT nach intravenöser Kontrastmittelgabe (Ausschnittsvergrößerung). Rechts paravertebrale Raumforderung, deren infraforaminaler Anteil zu einer Aufweitung des Foramen intervertebrale geführt hat 
bogens und der Aorta descendens sowohl im sagittalen als auch im seitlichen Thoraxübersichtsbild an Schärfe, sodass sich bei großen Raumforderungen mit entsprechend engem Kontakt die Grenzlinie zwischen Tumor und Aorta verliert.

Solange kein infiltratives Wachstum besteht, stellen sich Raumforderungen des hinteren Mediastinums praktisch immer als glatt begrenzte, rundlichovaläre, homogene Verdichtungen im Paravertebralraum dar. Projektionsradiograpisch ist eine Unterscheidung von zystischen und soliden Tumoren nicht möglich. Schwannome und Neurofibrome erstrecken sich in der Regel über etwa 2 Wirbelkörperhöhen. Ein Maximaldurchmesser über $5 \mathrm{~cm}$ ist insbesondere bei Patienten mit einer Neurofibromatose als Malignitätshinweis zu werten. Ganglioneurome sitzen der anterolateralen Zirkumferenz der Wirbelsäule breitbasig auf und sind bei Diagnosestellung häufig länger als 3 bis 5 Wirbelkörperhöhen. Neuroblastome führen $\mathrm{zu}$ ausgedehnten paraspinalen Verdichtungen.

Das Vorliegen oder Fehlen von Verkalkungen kann differentialdiagnostische Hinweise geben: Mediastinale Zysten sind im Gegensatz zu soliden Tumoren selten verkalkt. Im Kindesalter werden Verkalkungen gelegentlich bei primären (Neuroblastome), jedoch nie bei sekundären Mediastinaltumoren beobachtet. Im Erwachsenenalter sind verkalkte Ganglioneurino$m e$ beschrieben. Das Verkalkungsmuster liefert keine Hinweise auf die Dignität.

Wirbelsäule und Rippen werden sowohl von gutals auch von bösartigen Tumoren arrodiert. Durch länger bestehende Drucknekrosen können glatt begrenzte Defekte an Rippen, Wirbelkörpern und Neuroforamina entstehen. Unscharf begrenzte Knochendefekte sprechen für eine entzündliche oder maligne Genese. Ganglioneurome und Meningozelen/Meningomyelozelen können zu einer Skoliose führen.

Myelozelen und Meningomyelozelen lassen sich zwar myelographisch durch Kontrastierung der leptomeningealen Aussackung diagnostizieren, zur Artdiagnose und zur präoperativen Beschreibung der Ausdehnung sind jedoch eine Computertomographie (ggf. in Kombination mit einer intraspinalen Kontrastmittelgabe) oder eine MR-Tomographie indiziert.

\section{Schnittbilddiagnostik}

Die Aufgabe der Schnittbilddiagnostik besteht bei Tumoren des hinteren Mediastinums darin, die Artdiagnose durch eine Qualitätsbeschreibung der Tumormatrix und eine Bewertung der topographischen Pathoanatomie (Nachweis bzw. Ausschluss eines intraforaminalen/intraspinalen Tumoranteils) einzugrenzen (Bittner 1998; Chang 1994; Cifti 1998; Daldrup 1998; Ferguson 1998; Kawashima 1992; Ko 1998; Kohman 1993; Lange 1996; Lesko 1999; Merten
1991; Murray 1992; Schaefer-Prokop 1998; Schulman 1998; Siegel 1996; Taki 1996; Wegener 1992; Wilson 1994). Aufgrund der variablen Schichtführung und des höheren Weichteilkontrastes ist die MR-Tomographie der Computertomographie in der Darstellung der Weichteilkomponenten überlegen (Abb. $17.25 \mathrm{e}-\mathrm{g})$. Methodischer Vorteil der Computertomographie ist die bessere Darstellung ossärer Strukturen (Abb. 17.26-17.28).

Solide neurogene Tumoren finden ihre Entsprechung in paravertebralen, rundlich-ovalären Raumforderungen (Abb.17.28). Benigne Tumoren sind glatt begrenzt, eine unscharfe Berandung weist auf Malignität hin. Die Matrix neurogener Tumoren ist in der Regel homogen ausgebildet. Sie stellt sich computertomographisch überwiegend weichteiläquidens dar. Hypodensitäten entsprechen zellarmen Tumoranteilen, regressiv-zystischen Veränderungen oder Lipideinlagerungen. MR-tomographisch haben neurogene Tumoren in T1-Wichtung eine niedrige bis intermediäre, in T2-Wichtung eine intermediäre bis angehobene Signalintensität. Ganglioneurome und Neuroblastome zeigen in allen Sequenzen eine intermediäre Signalintensität. Pathognomonisches Zeichen eines Neurinoms/Neurofibroms ist eine sanduhrförmige Konfiguration, wobei der intraspinale Tumoranteil über einen im Neuroforamen gelegenen Stiel mit dem extraspinalen Tumoranteil verbunden ist. Mit Ausnahme des Neurinoms zeigen solide neurogene Tumoren in der Regel ein starkes Enhancement nach intravenöser Kontrastmittelgabe. Daher hebt sich ein intraspinaler Tumoranteil auf T1-gewichteten MR-Bildern nach intravenöser Kontrastmittelgabe gut von den physiologischen intraspinalen Weichteilstrukturen ab, wohingegen er nativdiagnostisch nur schwer vom gesunden Myelon (T1-Wichtung) bzw. vom intraspinalen Liquor (T2Wichtung) differenzierbar ist.

Bei soliden neurogenen Tumoren lassen sich in wechselndem Prozentsatz Tumorverkalkungen nachweisen, die jedoch nicht zur Sicherung der Artdiagnose beitragen. Erweiterte Neuroforamina, glatt berandete Wirbelkörper- und glatt begrenzte Rippenarrosionen werden als Ausdruck von Drucknekrosen durch länger bestehende intraforaminale Tumoranteile beobachtet. Unscharf berandete Skelettveränderungen weisen auf einen malignen Tumor hin. Die Artdiagnose muss jedoch bei therapeutischer Relevanz bioptisch geklärt werden.

Zystische neurogene Tumoren stellen sich als paravertebral gelegene, glatt begrenzte, rundliche oder ellipsoide zystische Raumforderungen dar. Die Dichte bzw. Signalintensität des Zysteninhalts entspricht in der Regel der des Liquors. Der Nachweis einer leptomeningealen Verbindung über ein meist erweitertes Foramen intervertebrale ist diagnoseweisend. Die 
Myelo-CT kann durch den Nachweis eines Übertritts von intrathekal appliziertem Kontrastmittel in die Zyste die Artdiagnose sichern.

\subsection{3}

\section{Sekundäre Mediastinaltumoren}

Unter sekundären Mediastinaltumoren werden lymphogene und hämatogene mediastinale Metastasen karzinomatöser und sarkomatöser Tumoren und ein mediastinaler Befall im Rahmen einer malignen lymphatischen Systemerkrankung subsummiert (Chang 1994; Daldrup 1998; Kohman 1993; Lackner 1998; Lange 1996; Leppert 1998; Manegold 1991; Schaefer-Prokop 1998; Wegener 1992). In der Mehrzahl der Fälle sind die mediastinalen Lymphknoten Ausgangspunkt des Tumorwachstums. Hämatogene Metastasierungen in Herz, Thymus und intrathorakale Strumen sind selten.

\section{Pathologie}

- Lymphknotenmetastasen. Lymphknotenmetastasen sind bei weitem die häufigste Ursache einer mediastinalen Raumforderung (Tabelle 17.1; Chang 1994; Hofmann 1991; Kohman 1993; Lange 1996; Leppert 1998; Manegold 1991; Schaefer-Prokop 1998; Wegener 1992).

Intrathorakale Tumoren wie das Bronchialkarzinom und das Ösophaguskarzinom metastasieren antegrad über ihre Lymphabflusswege in die abhängigen mediastinalen Lymphknotenstationen. Die weitere Metastasierung erfolgt über Anastomosen in nicht-abhängige mediastinale und kontralaterale hiläre Lymphknoten sowie antegrad über den D. thoracicus in die linke V. brachiocephalica.

In das Mediastinum metastasierende extrathorakale Tumoren sind mit abnehmender Häufigkeit im Magen, im Pankreas, in der Mamma, den Nieren, dem Hoden, der Prostata, der Schilddrüse oder dem Larynx lokalisiert. Folgende Ausbreitungswege sind beschrieben:

- Hämatogene Mikrometastasen in den Lungenkapillaren gelangen über eine Migration durch die Gefäßwand in die peripheren pulmonalen Lymphwege. Die weitere Ausbreitung erfolgt antegrad entlang der peribronchialen Lymphgefäße in die hilären und mediastinalen Lymphknoten.

- Mikrometastasen gelangen retrograd über präformierte Lymphbahnen in die Lymphknoten des oberen (Larynxkarzinom, Schilddrüsenkarzinom), des vorderen (Mammakarzinom) und des mittleren und hinteren Mediastinums (Magen-, Pankreasund Nierenzellkarzinom, maligne Hodentumoren). Sarkome metastasieren im Vergleich zu Karzinomen selten lymphogen in das Mediastinum.
- Maligne Lymphome. Sie stellen bei Erwachsenen mit etwa $20 \%$ den zweithäufigsten mediastinalen Tumortyp dar. Maligne Lymphome können isoliert im Mediastinum entstehen (Tabelle 17.1; s. Abschn. 17.4.2) oder als mediastinale Beteiligung einer generalisierten Lymphknotenerkrankung in Erscheinung treten. Sowohl die mediastinalen Lymphknoten als auch der Thymus können betroffen sein. Ein Mediastinalbefall liegt in zwei Dritteln der Erstdiagnosen eines M. Hodgkin und in einem Drittel der Erstdiagnosen eines Non-Hodgkin-Lymphoms vor.

Der M. Hodgkin ist mit über $50 \%$ der bioptisch gesicherten Fälle das häufigste mediastinale maligne Lymphom. In der Mehrzahl handelt es sich um den nodulär-sklerosierenden Typ. Typisch ist eine Lymphomausbreitung per continuitatem. Ein mediastinaler Hodgkin-Befall ist dementsprechend häufig mit supraklavikulären und zervikalen Lymphomen assoziiert. In bis zu $15 \%$ der Fälle lässt sich eine extranodale Infiltration der Umgebungsstrukturen nachweisen.

Die Non-Hodgkin-Lymphome stellen eine histologisch heterogene Gruppe dar, die histopathologisch unterschiedlich klassifiziert wird. Geläufig sind die europäische Kiel-, die US-amerikanische Rappaportund - neuerdings - die R.E.A.L.-Klassifikation, die niedriggradige, mittelgradige und hochgradige Lymphomformen unterscheiden (Tabelle 17.7; Hiddemann 1996; Meusers 1997). Eine multifokale Genese wird angenommen. Im Vergleich zum M. Hodgkin lässt sich ein höherer Anteil an extranodalem Tumorwachstum und ein unterschiedliches Befallsmuster feststellen. Non-Hodgkin-Lymphome breiten sich hämatogen, d.h. diskontinuierlich aus. Hierbei wird das Mediastinum häufig übersprungen („mediastinal skip“). Obwohl bei der Erstdiagnose von NonHodgkin-Lymphomen bereits in $80 \%$ mehrere Lymphknotenregionen befallen sind, entwickelt sich im Rahmen einer Generalisation nur in $10-15 \%$ der Fälle ein größerer Mediastinaltumor. Non-HodgkinLymphome mit mediastinaler „bulky disease " betreffen überwiegend die histologischen Subtypen mit hoher Malignität. Bei Kindern handelt es sich in mehr als der Hälfte der Fälle um leukämische oder aleukämische T-lymphoblastische Lymphome. Bei Erwachsenen dominieren die hochmalignen B-ZellLymphome. Maligne Non-Hodgkin-Lymphome sind am häufigsten im mittleren Mediastinum lokalisiert. Bei den leukämischen Verlaufsformen finden sich in einem Viertel der Fälle mediastinale oder hiläre Lymphome, vor allem bei der lymphozytären Form.

Unter Radiochemotherapie werden Größenabnahmen, nekrotische Einschmelzungen und/oder regressive Verkalkungen beobachtet. Bei soliden Restbefunden nach therapeutischer Intervention bereitet die Unterscheidung zwischen narbigen Abheilungen 
Tabelle 17.7. R.E.A.L.-Klassifikation der Non-Hodgkin-Lymphome

\begin{tabular}{|c|c|}
\hline B-Zell-Linie & T-Zell-Linie \\
\hline \multicolumn{2}{|l|}{ I. Niedriggradige NHL } \\
\hline $\begin{array}{l}\text { B-CLL } \\
\text { Lymphoplasmozytisches Lymphom } \\
\text { Haarzellenleukämie } \\
\text { MALT-Lymphom } \\
\text { Follikuläres Keimzentrumslymphom }\end{array}$ & $\begin{array}{l}\text { T-CLL } \\
\text { T-Zell-/NK-Zell-Typ der Lymphozytenleukämie } \\
\text { Mycosis fungoides } \\
\text { Sezary-Syndrom }\end{array}$ \\
\hline \multicolumn{2}{|l|}{ II. Hoch- bis mittelgradige NHL } \\
\hline $\begin{array}{l}\text { Promyelozytenleukämie } \\
\text { Plasmozytom } \\
\text { Mantelzell-Lymphom } \\
\text { Großzelliges follikuläres Keimzentrumslymphom } \\
\text { Diffuse großzellige B-Zell-Lymphome }\end{array}$ & $\begin{array}{l}\text { Prolymphozytenleukämie } \\
\text { Periphere T-Zell-Leukämie } \\
\text { Angioimmunoblastisches T-Zell-Lymphom } \\
\text { Angiozentrisches Lymphom } \\
\text { Intestinales T-Zell-Lymphom } \\
\text { Anaplastisches großzelliges Lymphom }\end{array}$ \\
\hline \multicolumn{2}{|l|}{ III. Hochgradige, sehr aggressive NHL } \\
\hline $\begin{array}{l}\text { Vorläufer B-lymphoblastisches Lymphom } \\
\text { Burkitt-Lymphom } \\
\text { B-Zellen-Leukämie } \\
\text { Plasmazellenleukämie }\end{array}$ & $\begin{array}{l}\text { Vorläufer T-lymphoblastisches Lymphom } \\
\text { Adultes T-Zell-Lymphom (ATLL) }\end{array}$ \\
\hline
\end{tabular}

und aktivem Restlymphomgewebe Schwierigkeiten. Auch eine Größenkonstanz bei längeren Verlaufskontrollen schließt aktives Lymphomgewebe innerhalb des Resttumors nicht aus. Eine solche Konstellation soll bei etwa $90 \%$ der Patienten mit mediastinalem Hodgkin- und bei etwa $40 \%$ der Patienten mit mediastinalem Non-Hodgkin-Befall gegeben sein. Etwa $50 \%$ der betroffenen Patienten erleiden im weiteren Verlauf ein Lymphomrezidiv.

\section{Klinik}

Entsprechend den Verhältnissen bei primären $\mathrm{Me}$ diastinaltumoren (Abschn. 17.4.2) sind mediastinale Lymphknotenmetastasen und Lymphome überwiegend asymptomatisch (Kohman 1993; Lange 1996; Leppert 1998; Manegold 1991; Schaefer-Prokop 1998; Wegener 1992). Nur bei Kompression oder Infiltration der entsprechenden anatomischen Leitstrukturen treten Dyspnoe (zentrale Atemwegsobstruktion), Dysphagie (Ösophagusobstruktion), Heiserkeit (Parese des N. recurrens), neuralgische Beschwerden (Nervenkompression, -infiltration) und Innervationsstörungen des Zwerchfells (Kompression oder Infiltration des N. phrenicus) auf.

Als Komplikationen mediastinaler Lymphknotenneoplasien werden obere Einflussstauungen, nekrotisierende bakterielle Pneumonien, Pilzpneumonien und atypische virale Pneumonien beschrieben. Der M. Hodgkin hat eine biphasische Altersverteilung mit einem ersten Gipfel um das 30. und einem zweiten um das 70. Lebensjahr. Das lymphatische Lymphoblastom tritt meist in der Kindheit, die anderen Non-Hodgkin-Lymphome am häufigsten im sechsten Lebensjahrzehnt auf.
Die N-Klassifikationen solider Organtumoren (Spiessl 1998) trägt dem individuellen Stadium der lymphogenen Ausbreitung Rechnung. Die Prognose mediastinaler maligner Lymphome richtet sich nach histologischem Subtyp und dem Krankheitsstadium. Die Stadieneinteilung des M. Hodgkin wird nach der Ann-Arbor-Klassifikation vorgenommen, in die die Anzahl der befallenen Lymphknoten, ihre Lokalisation in Bezug auf das Zwerchfell sowie die Beteiligung von Milz und extralymphatischen Organen eingehen (Tabelle 17.8). Die gleiche Klassifikation wurde für die Stadieneinteilung der Non-Hodgkin-Lymphome übernommen, wobei zusätzlich zwischen einem primär nodalen und primär extranodalen Befall unterschieden wird.

\section{Projektionsradiographie}

Lymphknotenvergrößerungen sind die häufigste Ursache einer Mediastinalverbreiterung (Kohman 1993; Lackner 1998; Lange 1996; Manegold 1991; Murray 1992). In den frühen Stadien ist die Thoraxübersichtsaufnahme ohne pathologischen Befund. Größere - in der Regel $>2 \mathrm{~cm}$ große - Tumoren führen $\mathrm{zu}$ unterschiedlichen projektionsradiographischen Mustern: Gruppierte Lymphknotenvergrößerungen konfluieren zu einer polyzyklisch begrenzten mediastinalen Verdichtung. Bei anderer Tumorkonfiguration entsprechen die bildmorphologischen Befunde den für Primärtumoren im vorderen, mittleren und hinteren Mediastinum beschrieben Veränderungen (s. 17.4.2).

Aufgrund der geschilderten Metastasierungswege finden sich Lymphknotenmetastasen gehäuft im mittleren Mediastinum. Sie erreichen in der Regel 
Tabelle 17.8. Ann-Arbor-Klassifikation der Hodgkin-Lymphome

$\begin{array}{ll}\text { Stadium I/IE } & \text { Lokalisierter Befall eines extralymphatischen Organs/Bezirks } \\ \text { Stadium II } & \text { Befall von mehreren Lymphknotenregionen auf derselben Seite des Zwerchfells } \\ \text { II E } & \text { Lokalisierter Befall eines extralymphatischen Organs/Bezirks und Lymphknotenbefall } \\ \text { Stadium III } & \text { Befall von Lymphknoten auf beiden Seiten des Zwerchfells } \\ \text { III E } & \text { Zusätzlich lokalisierter Befall eines extralymphatischen Organs/Bezirks } \\ \text { III S } & \text { Zusätzlich Befall der Milz } \\ \text { Stadium IV } & \text { Diffuser Organbefall mit/ohne Lymphknotenbefall } \\ \text { Zusatz A } & \text { Ohne systemische Symptome } \\ \text { Zusatz B } & \text { Mit Fieber, Nachtschweiß, Juckreiz, Gewichtsverlust }\end{array}$

nicht die Größe von malignen Lymphomen und zeigen im Gegensatz zu malignen Lymphomen meist einen einseitigen, zumindest aber einen asymmetrischen Hilus- und Mediastinalbefall (Abb. 17.29, 17.30). Bei Hodgkin-Lymphomen führt der häufige primäre Befall der zervikalen Lymphknoten und die Ausbreitung per continuitatem zu einem bevorzugten Befall der Lymphknoten des oberen vorderen und mittleren Mediastinums, während die Lymphknoten des hinteren unteren Mediastinums fast immer ausgespart sind (Abb. 17.30). Isolierte hiläre Lymphome stellen eine Ausnahme dar. Insgesamt bietet das mediastinale Befallmuster jedoch keine Möglichkeit, zwischen Hodgkin- und Non-Hodgkin-Lymphomen zu differenzieren.

Aufgrund projektionsradiographischer Kriterien ist es gleichfalls nicht möglich, reaktiv-entzündliche, granulomatöse und neoplastische mediastinale Lymphknotenerkrankungen voneinander $\mathrm{zu}$ unterscheiden. Der Nachweis grobscholliger Verkalkungen macht eine postentzündliche bzw. granulomatöse Genese wahrscheinlich. Lymphknotenverkalkungen werden jedoch auch nach Radiochemotherapie beobachtet. Zusätzliche Verdichtungen in Projektion auf die zervikalen, supraklavikulären und axillären Lymphknotenstationen können die Differentialdiagnose einengen. Allein die klinische Gesamtsituation, pulmonale, pleurale und ossäre Begleitbefunde und die Tatsache, dass bei zunehmendem Tumordurchmesser der Anteil maligner Lymphknotenveränderungen zunimmt, erlauben eine Abstufung der Differentialdiagnosen.

\section{Schnittbilddiagnostik}

Die Computertomographie ist derzeit die Methode der Wahl zur Klärung des mediastinalen und hilären Lymphknotenstatus (Abb. 17.31 und 17.32) (Bonomo 1996; Cascade 1998; Lackner 1998; Lange 1996; Leppert 1998; Manegold 1991; Murray 1992; SchaeferProkop 1998; Wegener 1992; Wilson 1994). Der für die Dignitätseinschätzung relevante nodale Längsdurchmesser wird wegen seiner meist orthogonalen Aus-

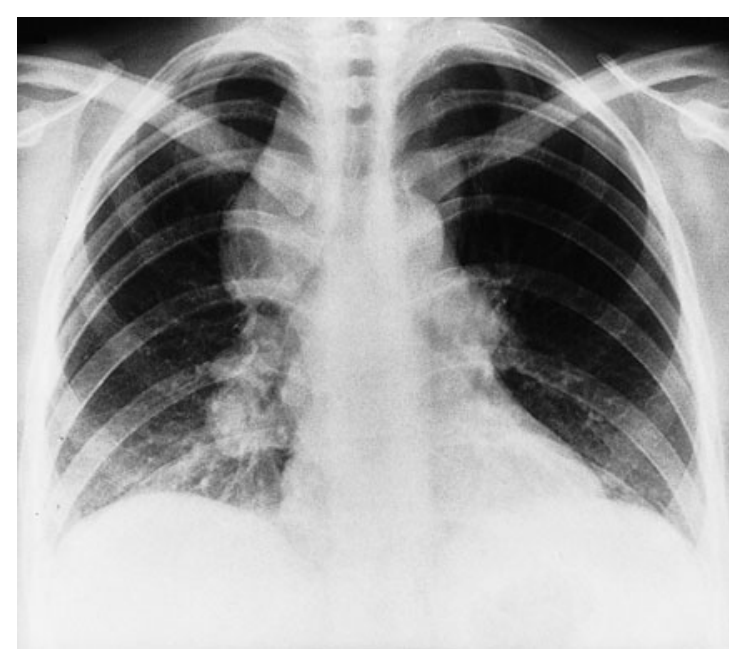

Abb. 17.29. Projektionsradiographische Darstellung von Lymphknotenmetastasen. Metastasierendes Bronchialkarzinom mit seitenasymmetrischer, polyzyklischer Mediastinalund Hilusbegrenzung

richtung zur transversalen Schichtebene computertomographisch nur mit der Spiralakquisitionstechnik zuverlässig erfasst.

Mit Ausnahme von Verlaufskontrollen während Radiochemotherapie liefert die MR-Tomographie keine überlegenen Ergebnisse (Bittner 1998; Lesko 1999; Siegel 1996). Die multiplanare Darstellungsmöglichkeit kann bei der Evaluierung der hilären, paratrachealen, aortopulmonalen und links paratrachealen Lymphknoten vorteilhaft sein (Abb. 17.33). Gleiches gilt für eine kontrastmittelunabhängige Differenzierung von Gefäß- und Lymphknotenanschnitten. Nachteilig ist die momentan noch unterlegene Ortsauflösung, die zu der Verwechslung zweier direkt benachbarter normal großer Lymphknoten mit einem pathologisch vergrößerten Lymphknoten führen kann, und die fehlende Beurteilbarkeit nodaler Verkalkungen. Die Entwicklung spezieller Oberflächenspulen und die Einführung schnellerer, höher auflö- 

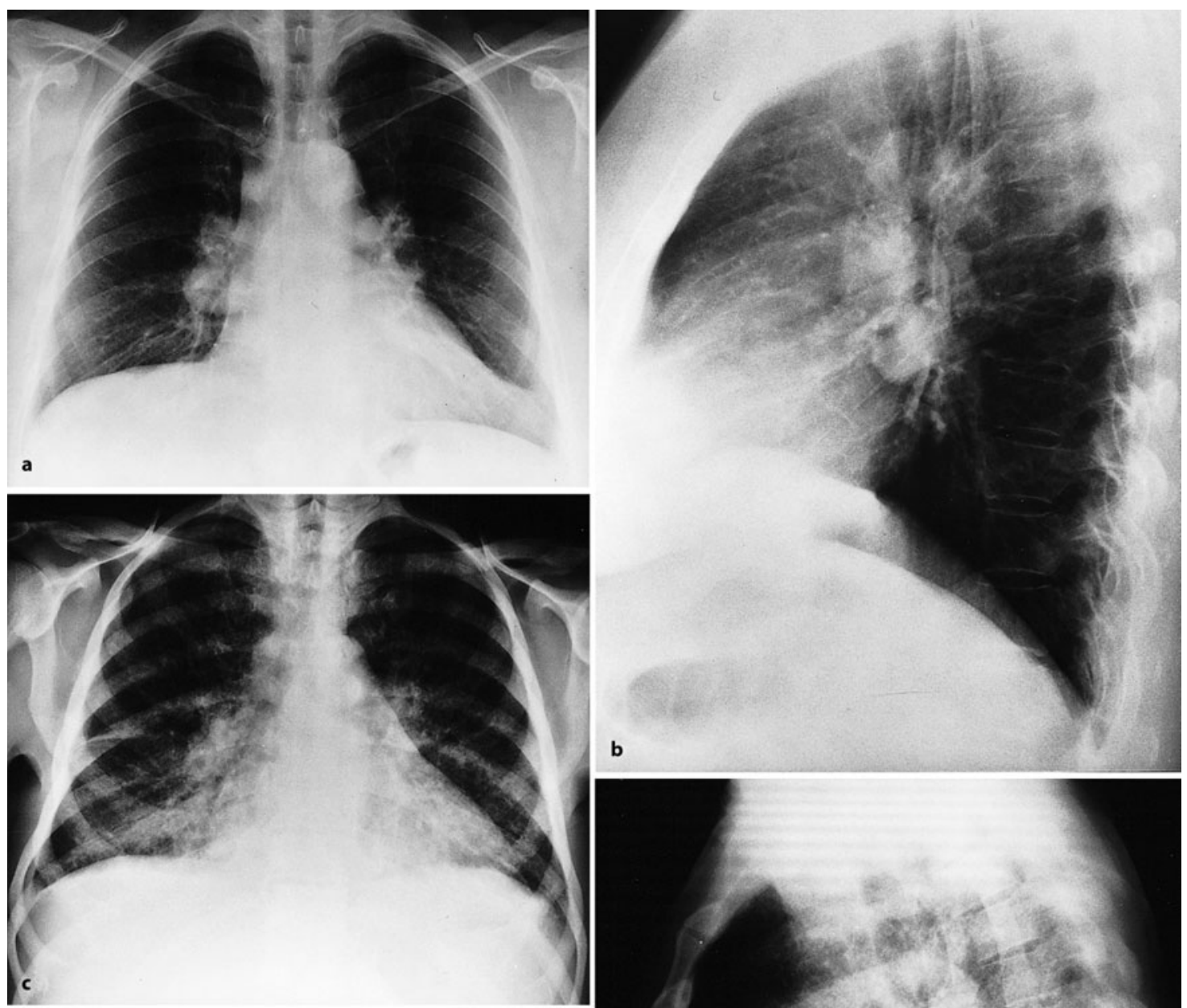

Abb. 17.30 a-d. Projektionsradiographische Darstellung systemischer Lymphknotenneoplasien. a, b Non-HodgkinLymphom mit beidseits hilären und mediastinalen Lymphomen auf der sagittalen (a) und seitlichen (b) Thoraxübersichtsaufnahme.c, d Akute myeloische Leukämie mit Befall der Lunge sowie der Hilus- und Azygoslymphknoten sichtbar auf der sagittalen (c) und seitlichen (d) Thoraxübersichtsaufnahme








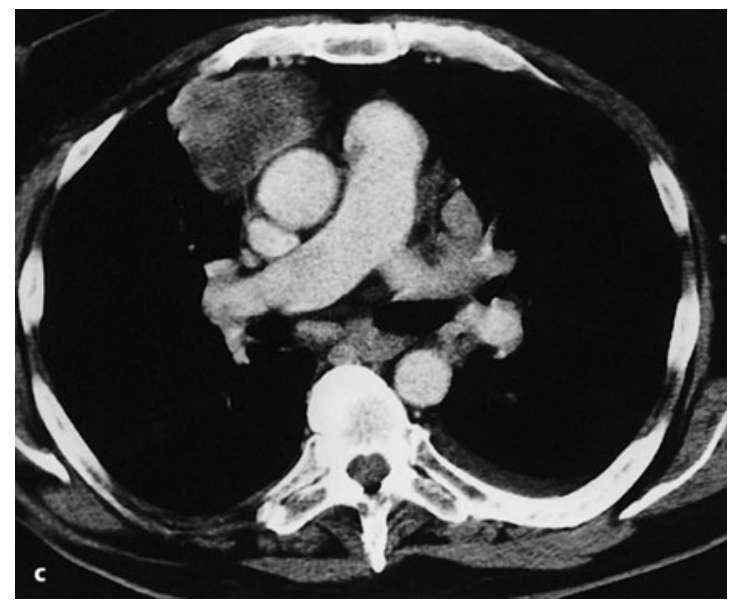

Abb. 17.31a-c. Computertomographische Darstellung von mediastinalen Lymphknotenmetastasen. a Rechts paratracheale, hypervaskularisierte Metastase eines Ösophaguskarzinoms, Nebenbefund: rechts deszendierende Aorta. b Der linken A. pulmonalis benachbarte, weniger stark vaskularisierte

senderer und weniger artefaktanfälliger Pulssequenzen versprechen in dieser Hinsicht Verbesserungen.

Da sich die Feinstruktur des nodalen Parenchyms bislang mit keinem bildgebenden Verfahren darstellen lässt, ist die Schichtbilddiagnostik in der Dignitätsbewertung auf die indirekten Kriterien Lokalisation, Form und Größe angewiesen (Cascade 1998; Chang 1994; Guyatt 1995). Vergrößerte perikardiale, retrosternale und retrokrurale Lymphknoten sind in jedem Fall malignomsuspekt. Lymphknoten im Abflussgebiet eines Malignoms (z.B. Bronchialkarzinom) sind mit höherer Wahrscheinlichkeit metastatisch befallen als solche außerhalb der lymphatischen Abflussstationen. Beidseitige mediastinale und hiläre Lymphknotenvergrößerungen weisen auf ein malignes Lymphom oder eine Sarkoidose hin.

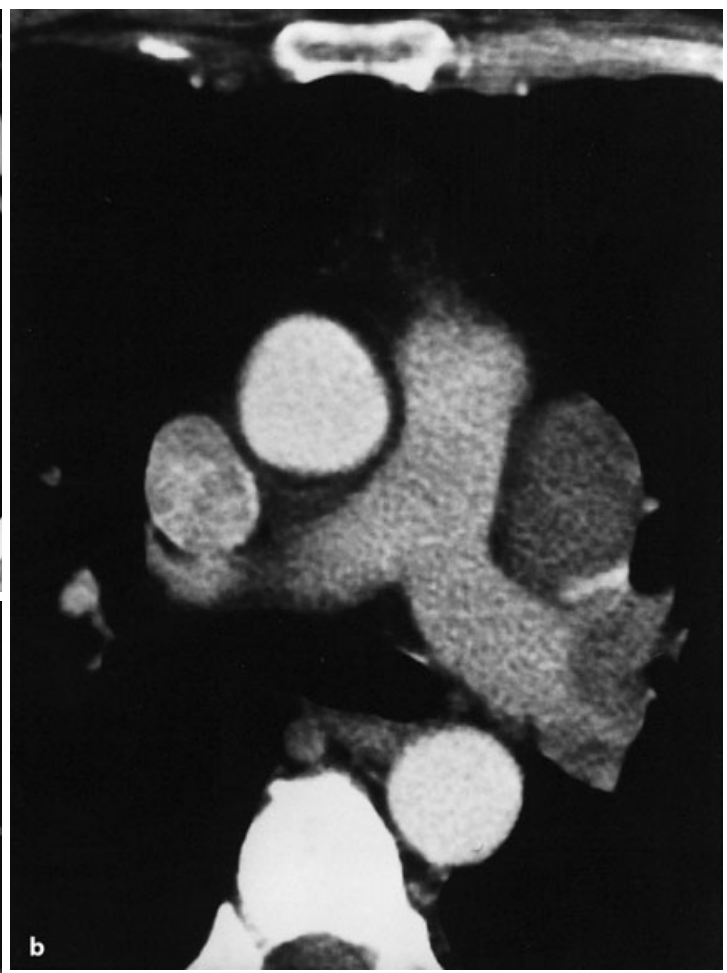

Metastasen eines Ösophaguskarzinoms bei einem anderen Patienten. c Metastatischer Konglomerattumor im vorderen Mediastinum und kleinere Lymphknotenmetastasen im mittleren und hinteren Mediastinum bei einem Patienten mit Seminom

Auch die Lymphknotenform erlaubt keine Dignitätszuordnung (Leppert 1998). Für normale Lymphknoten und frühe Pathologiestadien ist eine längsovale Form mit einem Verhältnis des maximalen Längs- (L) zum maximalen Querdurchmesser ( $\mathrm{T}$ ) von $\geq 2$ charakteristisch. Eine rundliche Lymphknotenform mit einem L/T-Quotienten $<2$ spricht für Entzündungen, Metastasen und maligne Lymphome.

Die Wahrscheinlichkeit einer Metastasierung nimmt exponentiell mit der Lymphknotengröße zu (Guyatt 1995; Leppert 1998). Ein Querdurchmesser $>10 \mathrm{~mm}$ wird allgemein als malignomsuspekt angesehen. Ausnahmen sind die Lymphknoten in Umgebung der Carina und der V. azygos, bei denen ein maximaler Querdurchmesser von bis zu $15 \mathrm{~mm}$ als normal gilt. Bei Dignitätseinstufungen, die auf der Bewertung der Lymphknotengröße basieren, handelt 


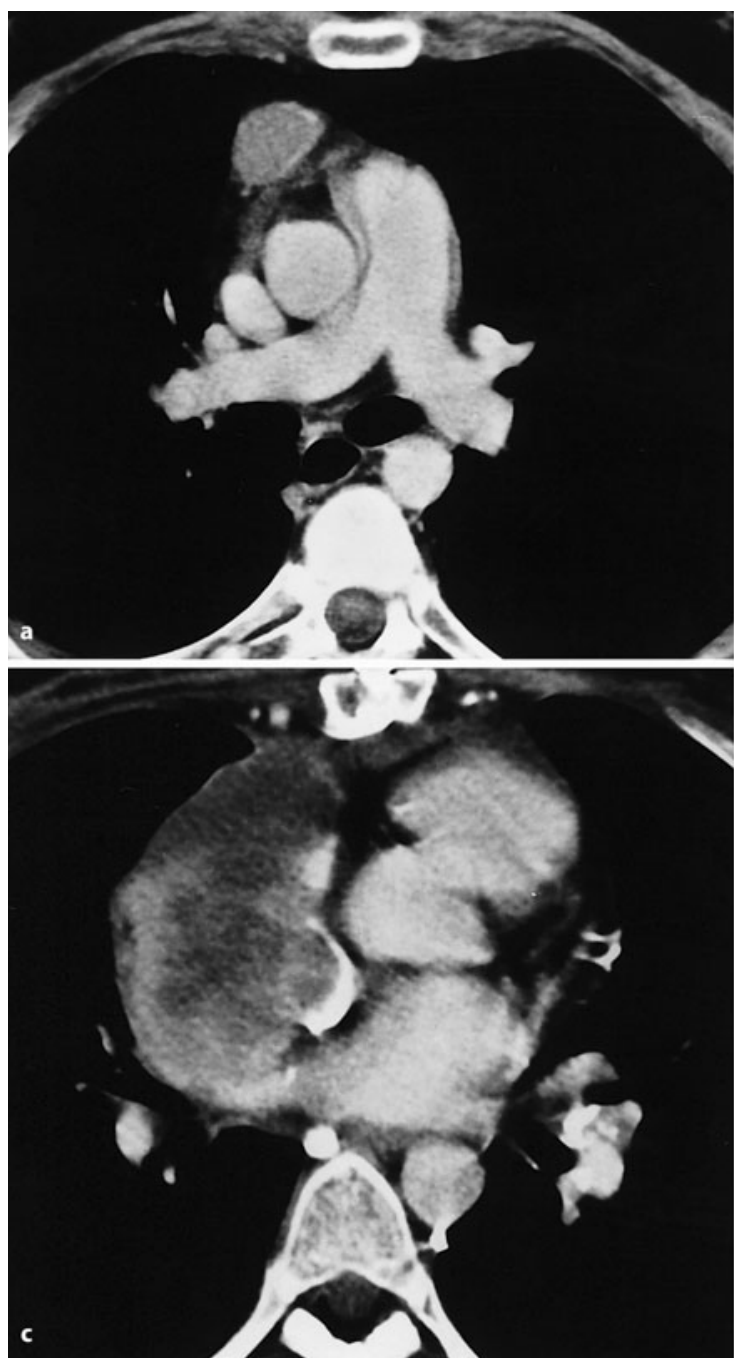

Abb. 17.32 a-d. Computertomographische Verlaufskontrollen von Hodgkin-Lymphomen unter Strahlentherapie (a, b) und unter Chemotherapie (c, d). a CT vor Radiatio mit Lymphomnachweis im vorderen Mediastinum und $\mathbf{b}$ nach Radiatio mit Nachweis von narbigen Residuen. c CT vor Chemotherapie
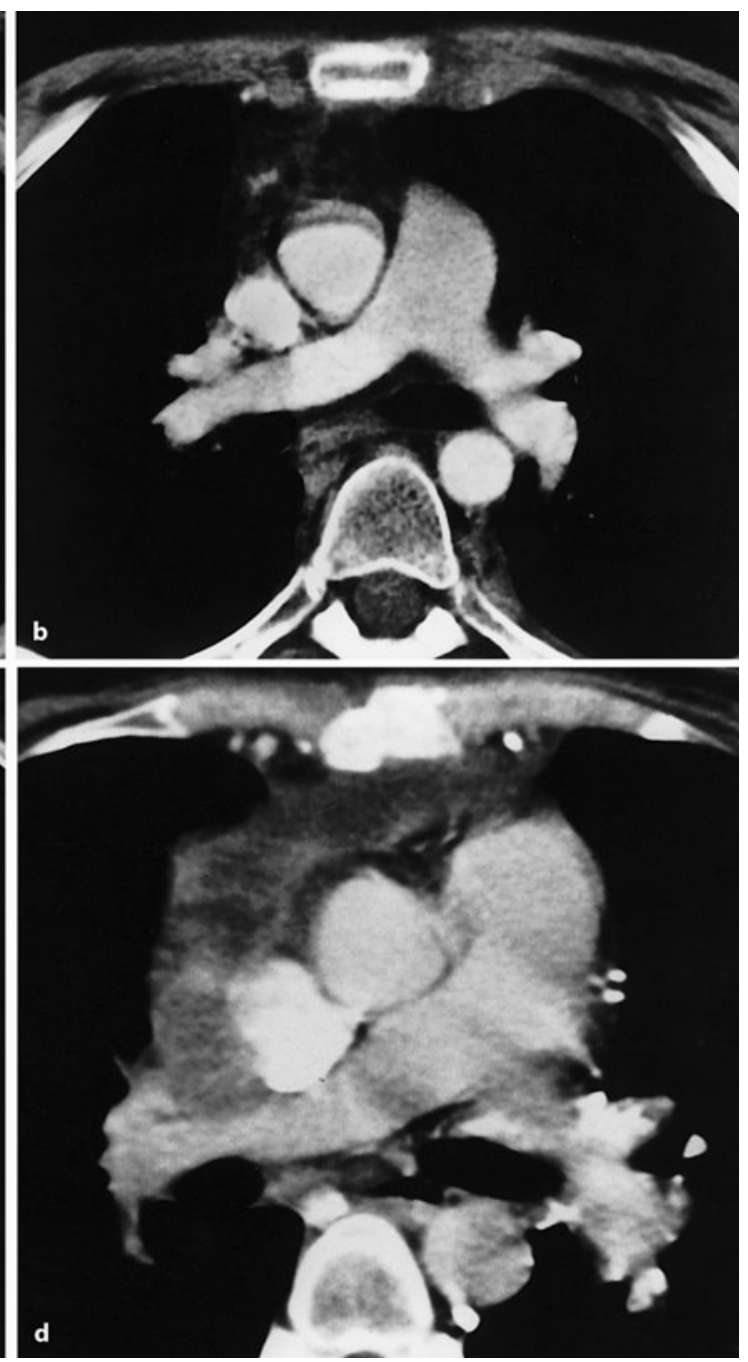

bei einem anderen Patienten mit einem Lymphom im vorderen und mittleren Mediastinum, das die V. cava superior komprimiert. d CT nach Chemotherapie mit Volumenreduktion des Tumors ohne Befundnormalisierung es sich jedoch nur um Wahrscheinlichkeitsangaben, da das Parenchym normal großer Lymphknoten in bis $\mathrm{zu} 15 \%$ Mikrometastasen enthält und mäßige Lymphknotenvergrößerungen in bis zu $30 \%$ der Fälle auf eine entzündliche Genese zurückzuführen sind. Insbesondere bei Begleitpneumonien ist an die letztgenannte Möglichkeit zu denken. Dementsprechend sind die Ergebnisse von Computertomographie und MR-Tomographie in der mediastinalen Lymphknotendiagnostik unbefriedigend: Wird der Schwellenwert auf einen maximalen Querdurchmesser von $10 \mathrm{~mm}$ festgesetzt, so wird für die Computer- tomographie eine Sensitivität von ca. $60 \%$ und eine Spezifität von ca. $70 \%$ für den Nachweis eine malignen Befalls angegeben.

Lymphknotenvergrößerungen finden ihre computer- und MR-tomographische Entsprechung in nodulären oder unstrukturierten Verdichtungen (Abb. 17.31, 17.32) bzw. Signalintensitätsänderungen (Abb. 17.33), die die normalen Mediastinalstrukturen maskieren (Gawne-Cain 1996; Leppert 1998; Schaefer-Prokop 1998; Slanetz 1998). Die computertomographische Densität neoplastisch befallener Lymphknoten liegt im Bereich von Weichteilgewebe 

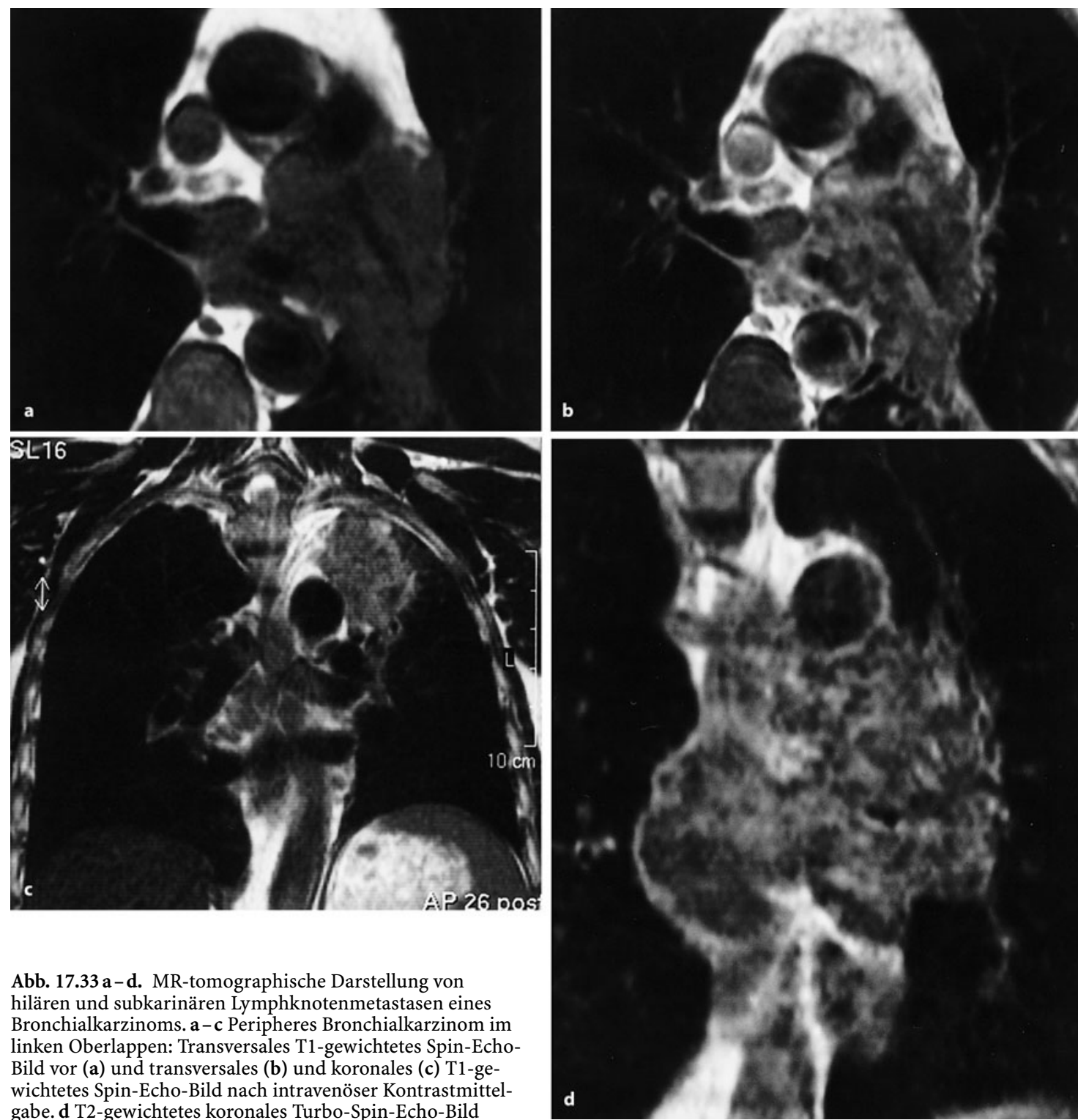

Abb. 17.33 a-d. MR-tomographische Darstellung von hilären und subkarinären Lymphknotenmetastasen eines Bronchialkarzinoms. a-c Peripheres Bronchialkarzinom im linken Oberlappen: Transversales T1-gewichtetes Spin-EchoBild vor (a) und transversales (b) und koronales (c) T1-gewichtetes Spin-Echo-Bild nach intravenöser Kontrastmittelgabe.d T2-gewichtetes koronales Turbo-Spin-Echo-Bild

oder gering darunter. Maligne Lymphome haben aufgrund ihres hohen Wasseranteils in der Regel eine lange T2-Zeit. Es kommen jedoch sowohl in T1- als auch insbesondere in T2-Wichtung Überlappungen der Signalintensitäten von tumorösen und entzündlichen Lymphknotenerkrankungen vor. Nicht behandelte nodulär sklerosierende Hodgkin-Lymphome zeichnen sich durch ein heterogenes Signalintensitätsmuster in T2-Wichtung aus. Eine niedrige Signalintensität in T2-Wichtung spricht für Fibrosen nach Radiochemotherapie. Zentrale Nekrosen können entzündlichen Einschmelzungen (Tuberkulose, Staphy-

lokokkeninfektion), ischämischen Kolliquationen bei neoplastisch befallenen Lymphknoten und einem Zustand nach Radiochemotherapie entsprechen. Da die MR-Bildgebung auf der Protonendichte der untersuchten Gewebe basiert, bietet die MR-Tomographie Vorteile bei der Dignitätsklärung von Resttumoren nach Radiochemotherapie: Residuale oder neu entstandene Lymphomzellen unterscheiden sich von Bindegewebszellen durch einen höheren Wasser- und einen niedrigeren Proteingehalt, sodass sich Lymphomgewebe ab einem bestimmten Volumen in T2-Wichtung signalreich von narbigem Bindegewebe abhebt. 
Ein Enhancement nach intravenöser Kontrastmittelgabe führt bei normalen Lymphknoten, reaktiv hyperplastischen Lymphknoten und malignen Lymphomen zu homogenen Signalanhebungen (bis zu 20 H.E. in der CT; Abb. 17.32 a, 17.33 b-d). Die sehr seltenen hypervaskularisierten Metastasen eines Nieren-, Schilddrüsen- oder kleinzelligen Bronchialkarzinoms zeigen ein stärkeres Enhancement (Abb. 17.31a). Ein inhomogenes Enhancement wird bei Lymphknotenmetastasen und Amyloidosen beobachtet. Ein peripheres Enhancement kennzeichnet zentral nekrotisierende Lymphknotentuberkulosen und Lymphknotenmetastasen. Erfolgversprechend scheinen MR-lymphographische Kontrastmittel aus ultrakleinen Eisenoxidpartikeln, die interstitiell oder transvenös verabreicht und von gesundem Lymphknotengewebe aufgenommen werden. Ihr Einsatz befindet sich z.Z.im Stadium der klinischen Erprobung (Okuhata 1994).

Die Dignitätsbestimmung mediastinaler Lymphknoten ist schnittbilddiagnostisch nur mit Einschränkungen möglich. Sowohl computer- als auch MR-tomographisch lässt sich bei fibrolipomatösen oder postentzündlich vernarbten Lymphknoten hiläres Vakatfett von regressiv verändertem Lymphknotenparenchym differenzieren. Da Neoplasien in aller Regel zu einer Obliteration des hilären Fettgewebes führen, gilt dessen Nachweis als verlässlicher Hinweis auf Benignität. Anamnese, Klinik und Begleitbefunde wie zervikale, supraklavikuläre, axilläre und subdiaphragmale Lymphknotenvergrößerungen und pulmonale, pleurale und ossäre Pathologien können die Differentialdiagnose einengen.

Nodale Verkalkungen sind nur selten Ausdruck knochenbildender Metastasen eines Osteosarkoms, eines Ovarialkarzinoms, eines bronchioalveolären Karzinoms oder eines radiojodtherapierten Schilddrüsenkarzinoms, sondern überwiegend Folge einer Radiochemotherapie oder einer abgeheilten Tuberkulose, Histoplasmose, Sarkoidose, Silikose oder Amyloidose (Abb. 17.34, 17.35 c; Gawne-Cain 1996). Starke Dichteanhebungen ohne aktuell vorangegangene Kontrastmittelgabe sprechen bei älteren Patienten und entsprechender Anamnese für einen Zustand nach Lymphographie.

Bei der Bildanalyse müssen Lymphknoten von anderen, im Schichtbild ebenfalls rundlich-ovalären Mediastinalstrukturen abgegrenzt werden. Eine transvenöse Kontrastierung der großen Gefäße und des Schilddrüsenparenchyms erleichtert die computertomographische Zuordnung von Queranschnitten der entsprechenden Strukturen. Anschnitte der Speiseröhre sind durch orale Kontrastierung des Lumens und die Analyse des Verlaufs der Speiseröhre über mehrere Schichtbilder eindeutig erkennbar. Die

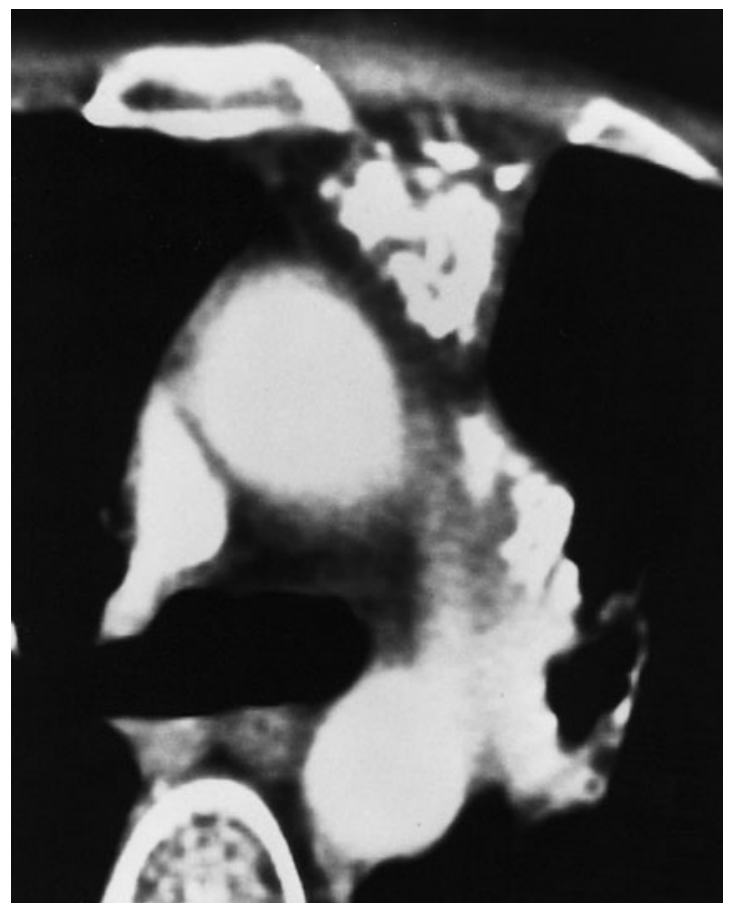

Abb. 17.34. Teilverkalkte Verdichtung im vorderen oberen Mediastinum als Residuum eines bestrahlten Hodgkin-Lymphoms. CT nach intravenöser Kontrastmittelgabe (Ausschnittsvergrößerung)



Abb. 17.35 a-c. Nodale Verkalkungen als Folge einer Silikose auf der sagittalen (a) und der seitlichen (b) Thoraxübersichtsaufnahme und als Folge einer Tuberkulose auf dem CT nach intravenöser Kontrastmittelgabe (c) 

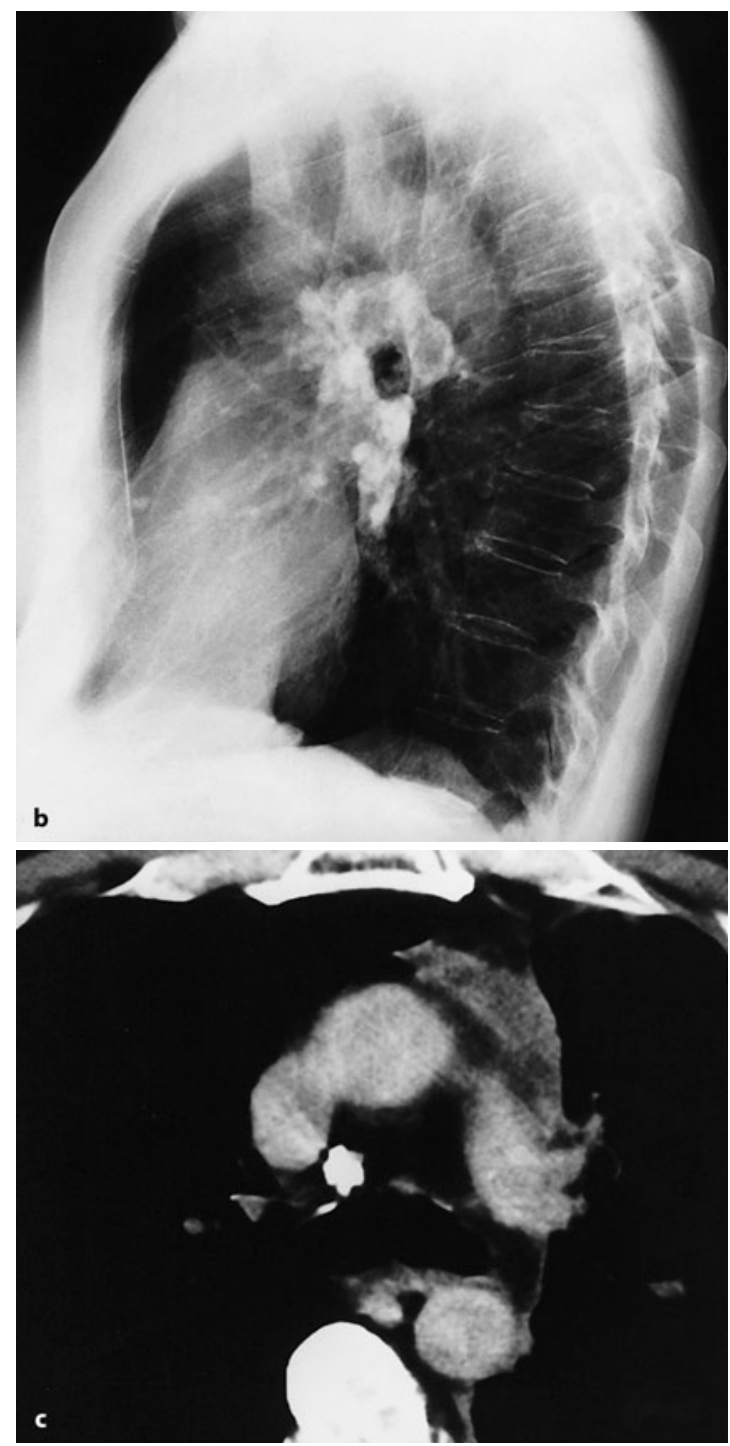

Abb. 17.35 b, c. Legende s. S. 42

obere perikardiale Umschlagfalte, die sich in $50 \%$ der Fälle dorsal der Aorta ascendens in Höhe der linken Pulmonalarterie darstellt, darf nicht als Lymphknoten fehlinterpretiert werden. Paravertebrale Venen und die Cisterna chyli können vergrößerte retrokrurale Lymphknoten vortäuschen. Die Cisterna chyli kann einen Querdurchmesser von bis $\mathrm{zu} 2 \mathrm{~cm}$ und einen Längsdurchmesser von bis $\mathrm{zu} 8 \mathrm{~cm}$ erreichen. Bei der Bildanalyse helfen die tubuläre Struktur, die typische Lage rechts lateral der Aorta in Höhe BWK 12/LWK 2 und die fett- bis flüssigkeitsäquivalente Dichte. Noduläre Zwerchfellverdickungen können Lymphknoten ähneln.

\subsection{4}

\section{Mediastinale Lymphadenopathien}

\section{Pathologie und Klinik}

Das Spektrum benigner Lymphknotenvergrößerungen reicht von unspezifischen reaktiven Hyperplasien, über die 1954 von Castleman beschriebene angiofollikuläre Hyperplasie, entzündliche (bakterielle, virale, mykoplasmatische, mykotische und parasitäre Infektionen) und granulomatöse Lymphknotenveränderungen (Sarkoidose) bis hin zu Lymphknotenveränderungen im Rahmen von Pneumokoniosen (Silikose, Asbestose) und Speicherkrankheiten (Amyloidose) (Baran 1996; Ecklund 1994; GawneCain 1996; Hainaut 1998; Hofmann 1991; Kohman 1993; Lange 1996; Moon u. Han 1994; Leppert 1998; Moon 1996; Schaefer-Prokop 1998; Siegel 1996; Wegener 1992).

Entsprechend dem breiten Ursachenspektrum sind die pathophysiologischen Mechanismen, die zu einer Lymphadenopathie führen, ebenso wie ihre klinischen Manifestationsformen vielgestaltig. In seltenen Fällen kann eine Methothrexat-, Diphenylhydantoin- oder Cyclosporintherapie Ursache einer mediastinalen Lymphadenopathie sein. In den übrigen Fällen ist die Lymphadenopathie in aller Regel Ausdruck einer systemischen oder intrathorakalen Erkrankung.

\section{Projektionsradiographie}

Die Lymphadenopathie findet ihre projektionsradiographische Entsprechung in hilären und mediastinalen Raumforderungen und Verdichtungen. Lymphadenopathien sind allein anhand bildmorphologischer Kriterien nicht von malignen Erkrankungen abzugrenzen (Kohman 1993; Lackner 1998; Lange 1996; Murray 1992). Oft sind jedoch Anamnese, Klinik und bildgebende Begleitbefunde richtungweisend, sodass sich eine histologische Diagnosesicherung erübrigt. Dies gilt insbesondere für die synoptische Betrachtung pulmonaler und hilär/ mediastinaler Veränderungen auf der Thoraxübersichtsaufnahme (Tabelle 17.9).

Differentialdiagnostische Hinweise liefern die Lokalisation (hilär vs. mediastinal) und die Form nodaler Veränderungen (symmetrisch vs. asymmetrisch) sowie das Vorliegen und die Form nodaler Verkalkungen. Entzündlich veränderte Lymphknoten finden sich im Bereich der Erregereintrittspforte bzw. in den Lymphknoten, die das erkrankte Organ drainieren. So werden bei der aktiven Tuberkulose in $80 \%$ der Fälle unilaterale und in $20 \%$ bilaterale Hilusvergrößerungen beobachtet. Typisch sind asymmetrische, polyzyklisch begrenzte hiläre und mediastinale Verdichtungen. Auch bei der akuten Histoplasmose, der akuten Toxoplasmose und der Silikose 


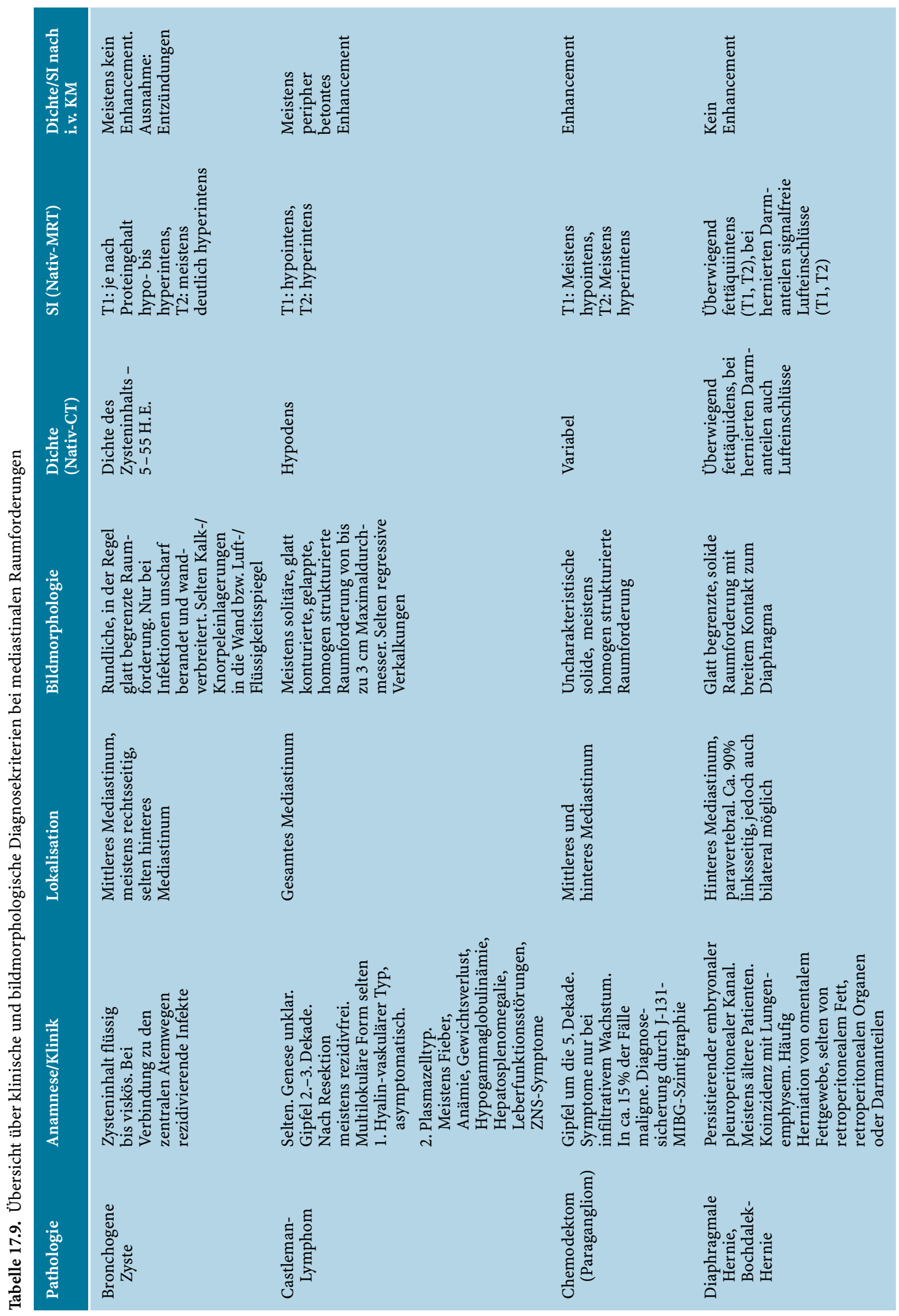




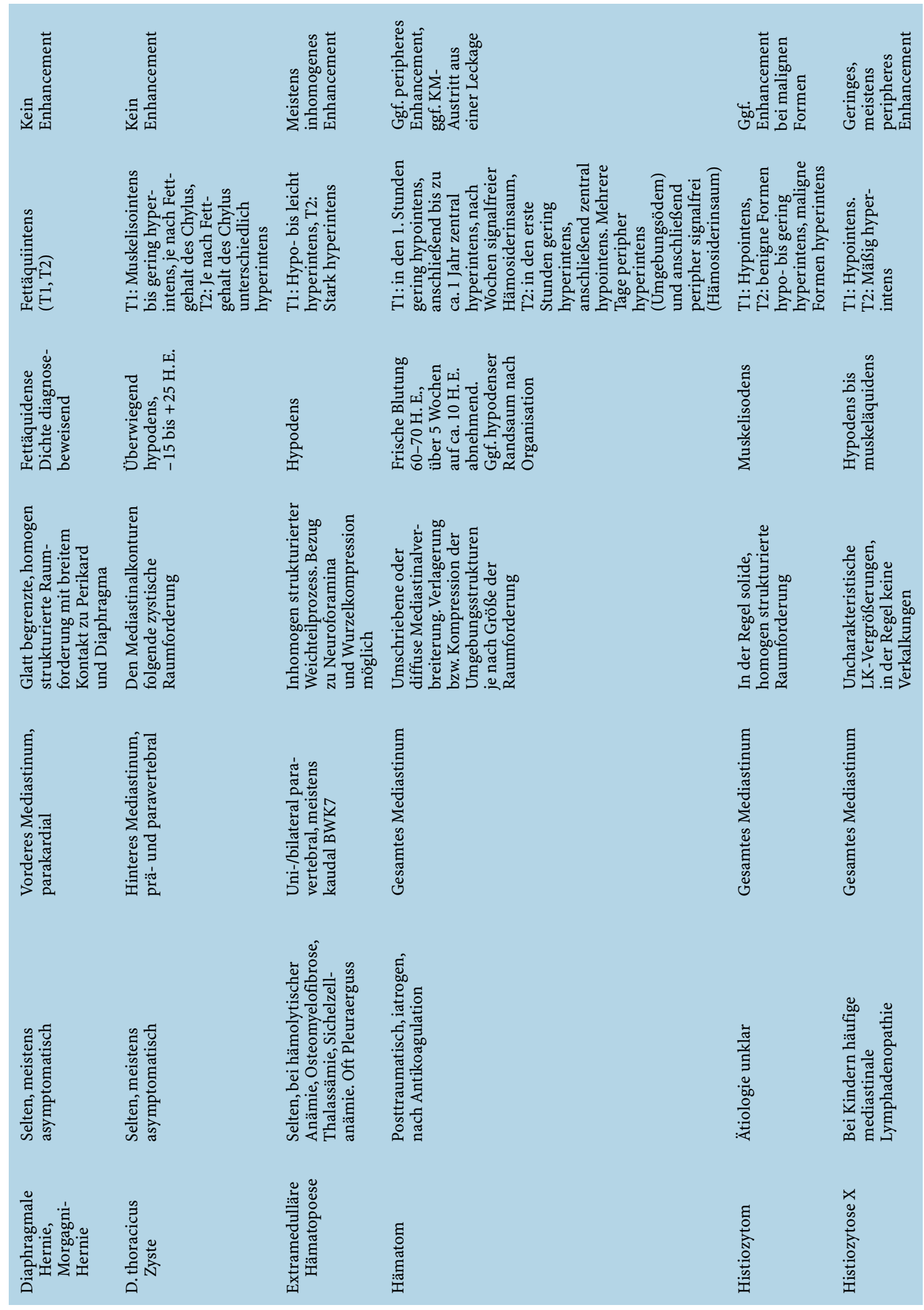




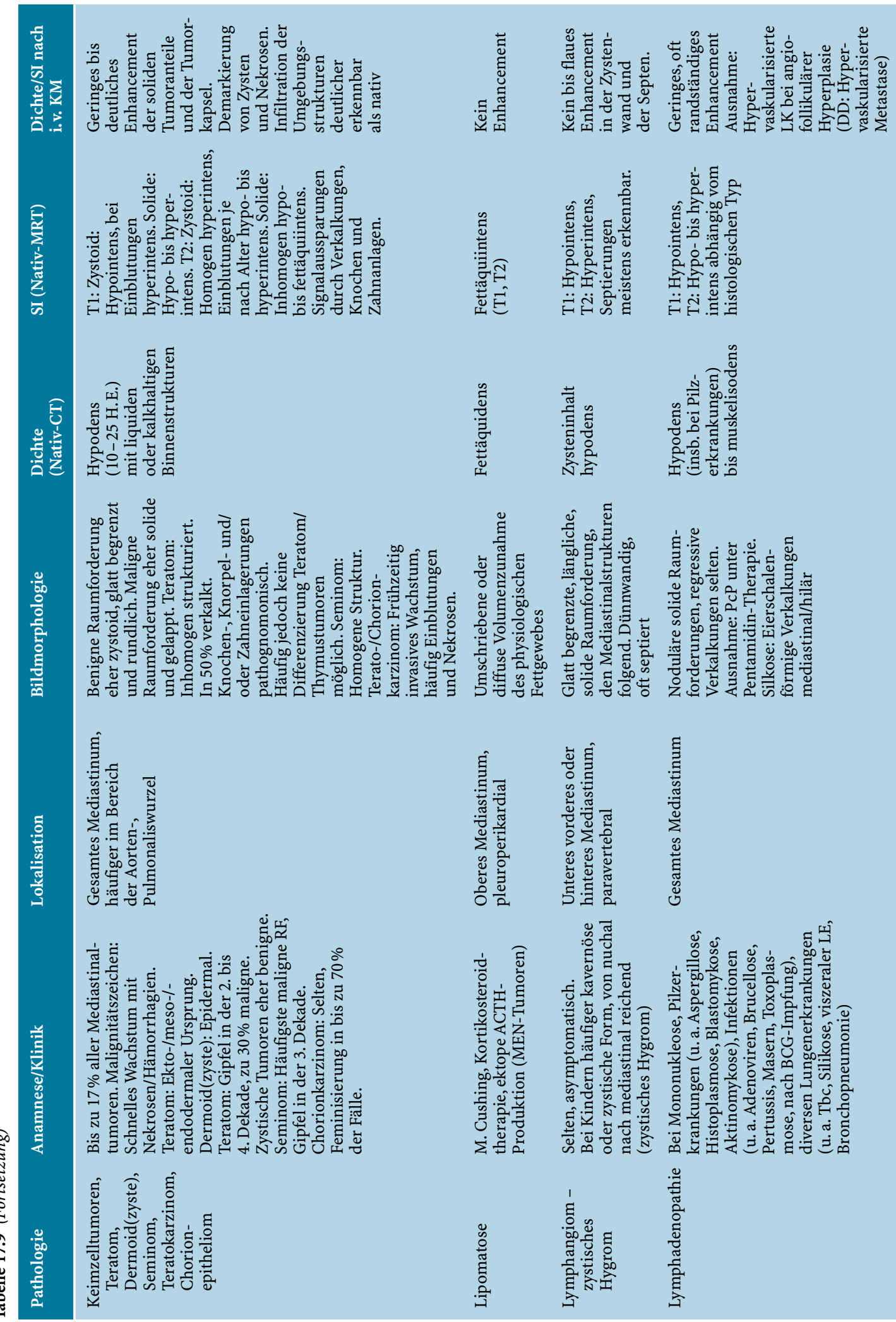




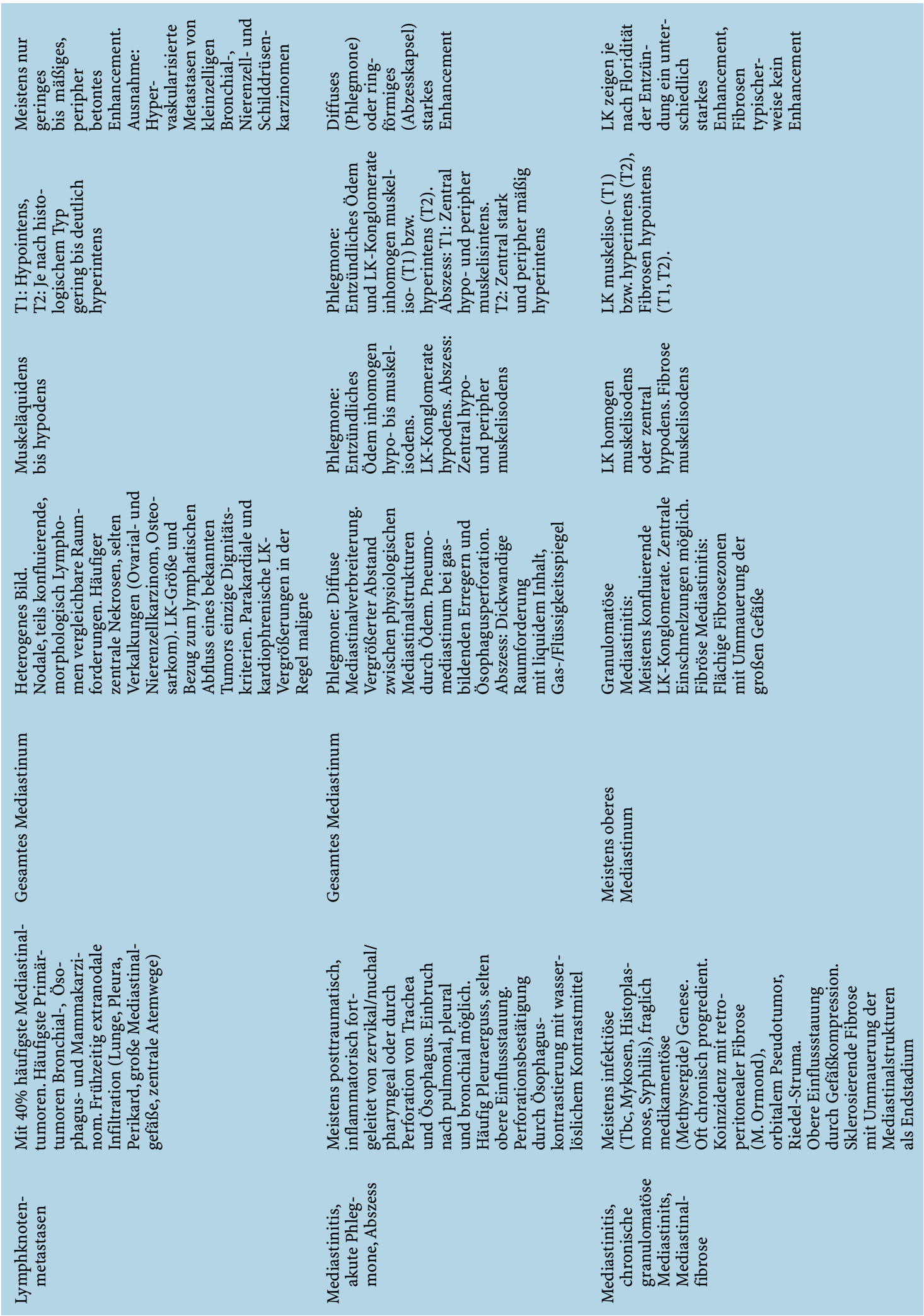




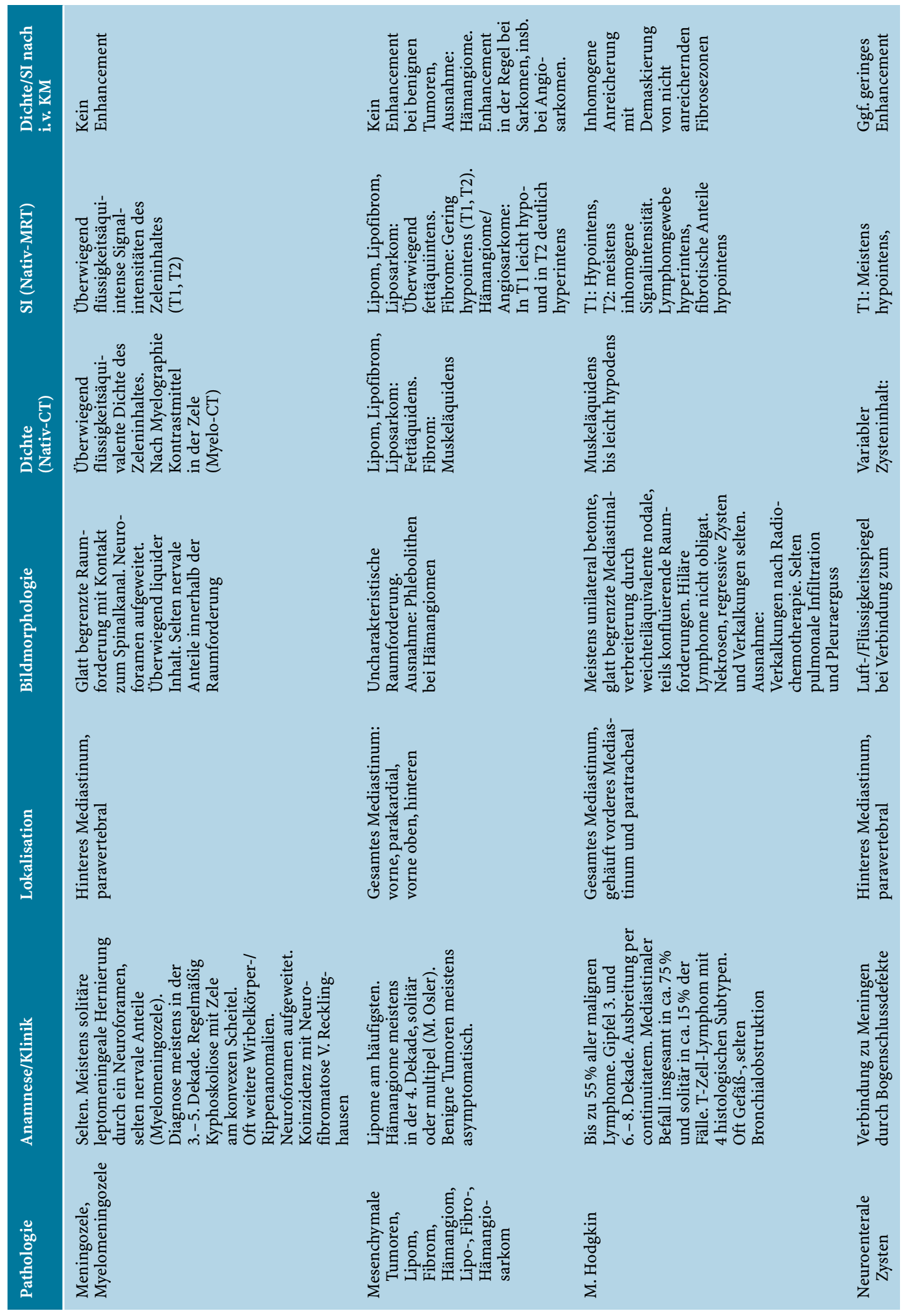




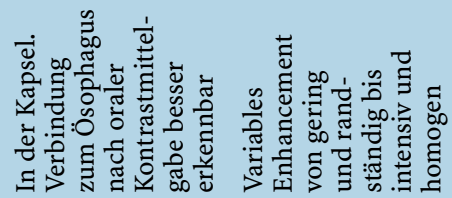

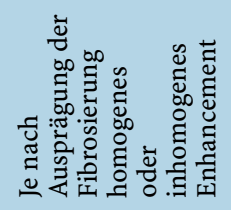

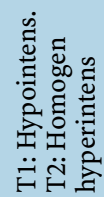

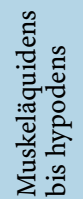
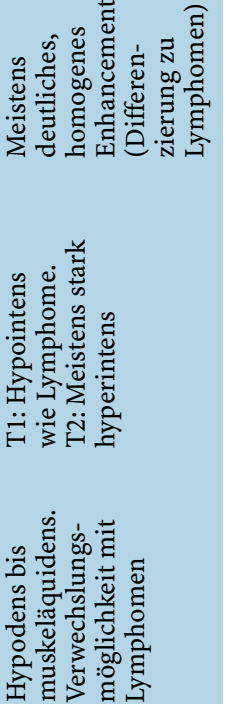

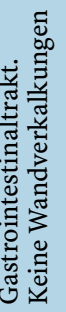
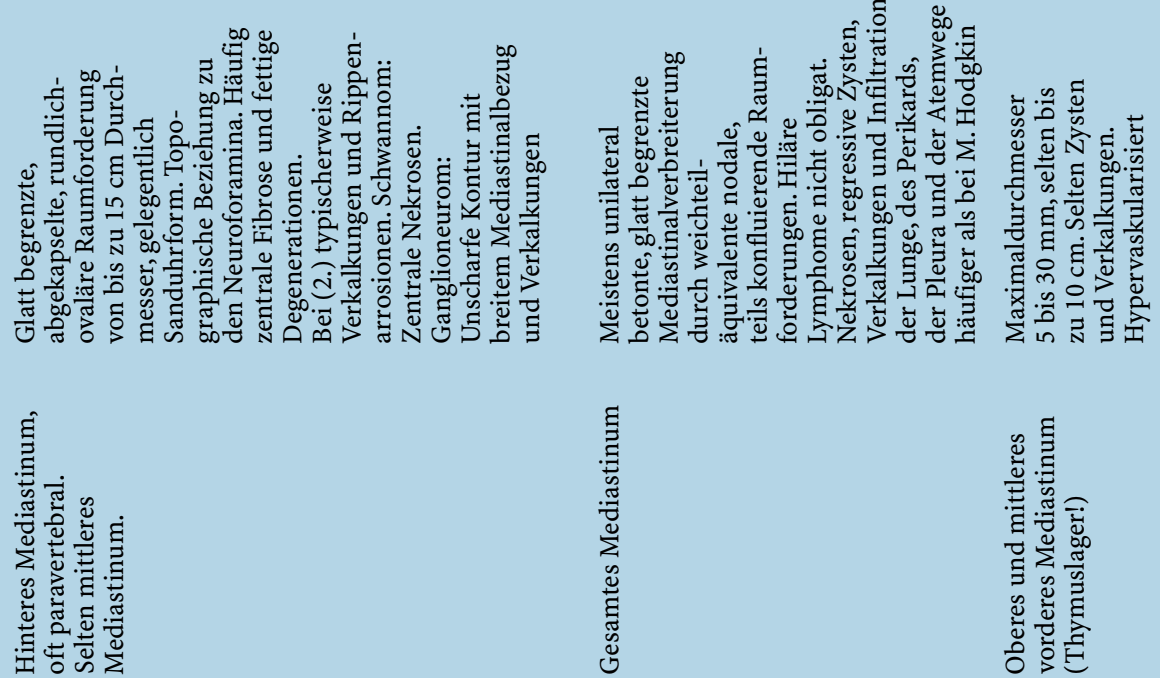

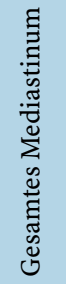
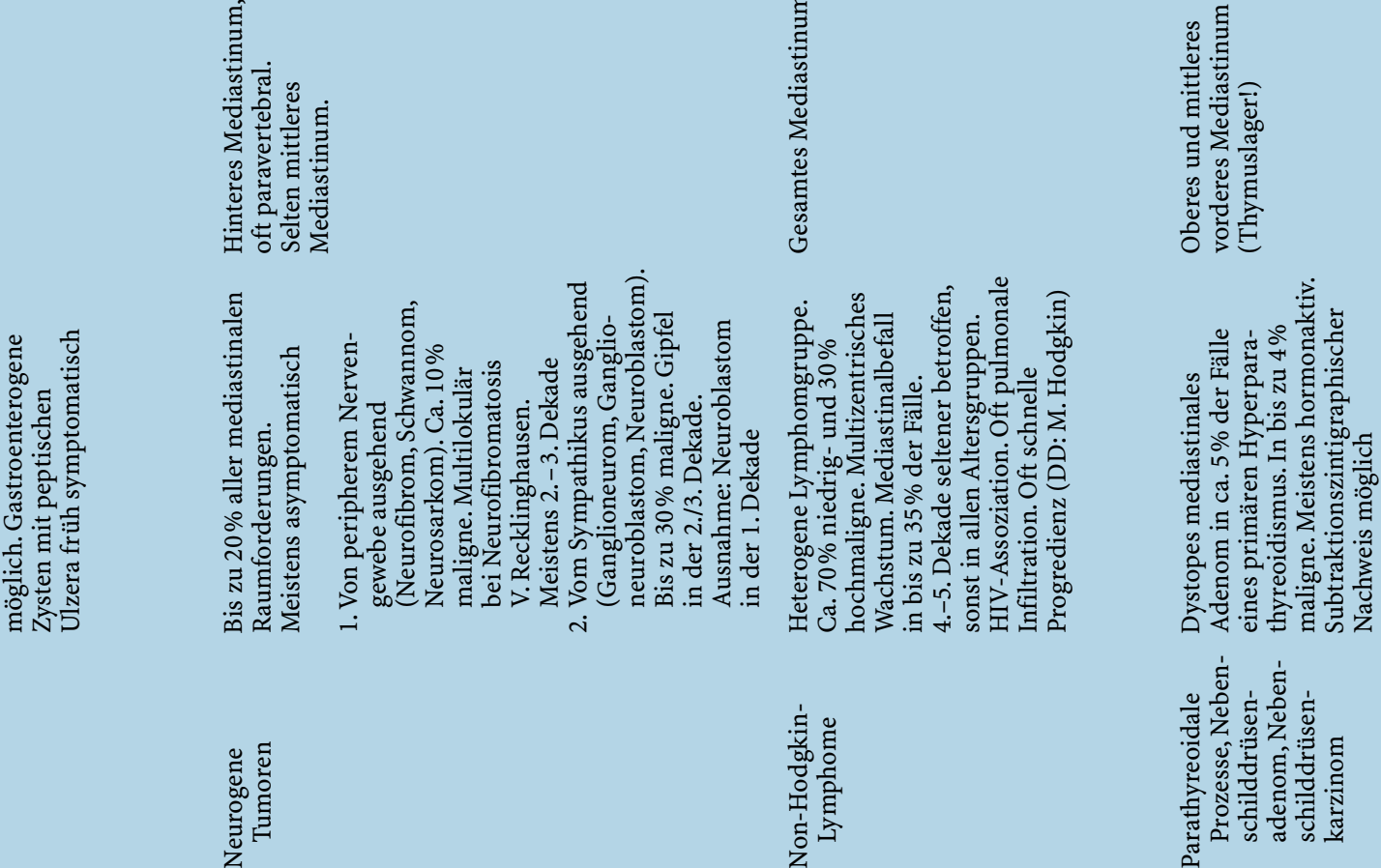


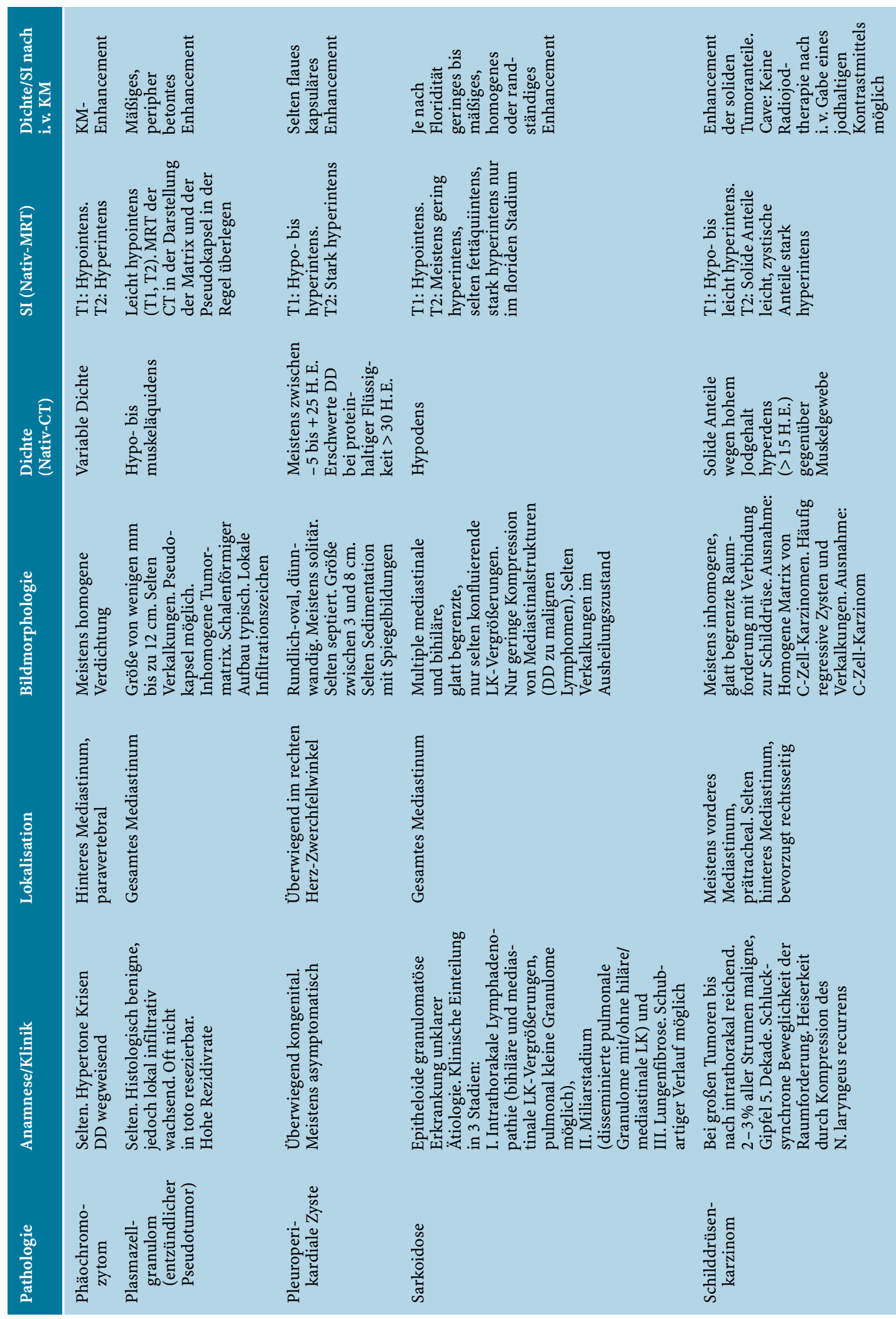




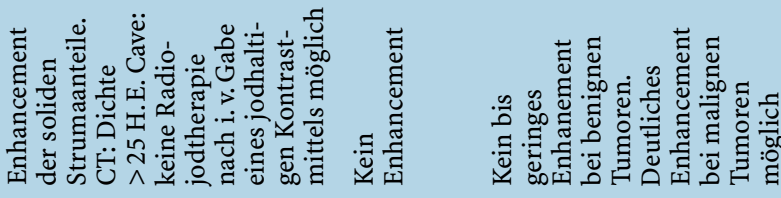

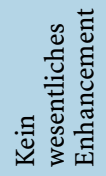
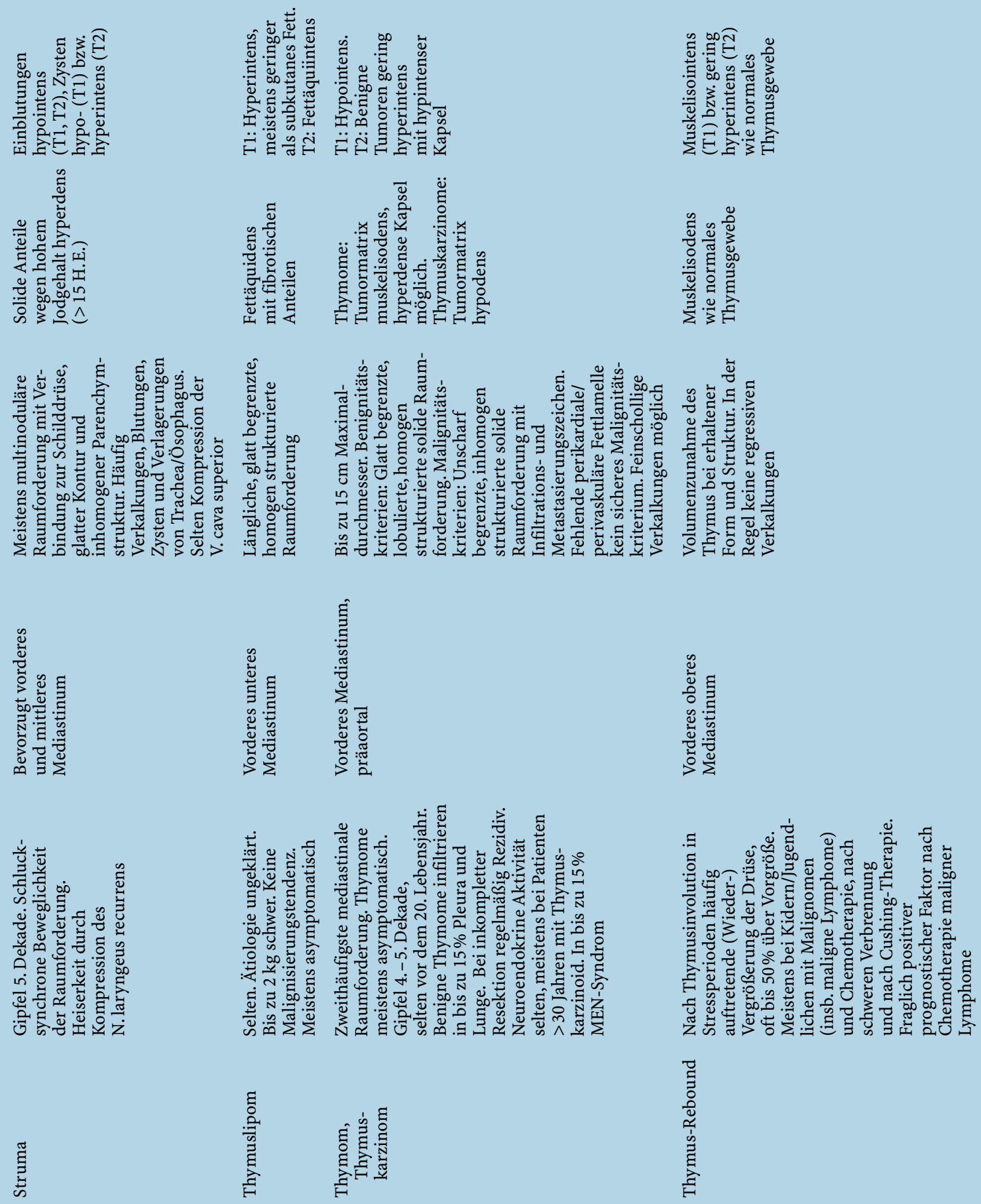

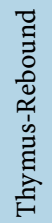



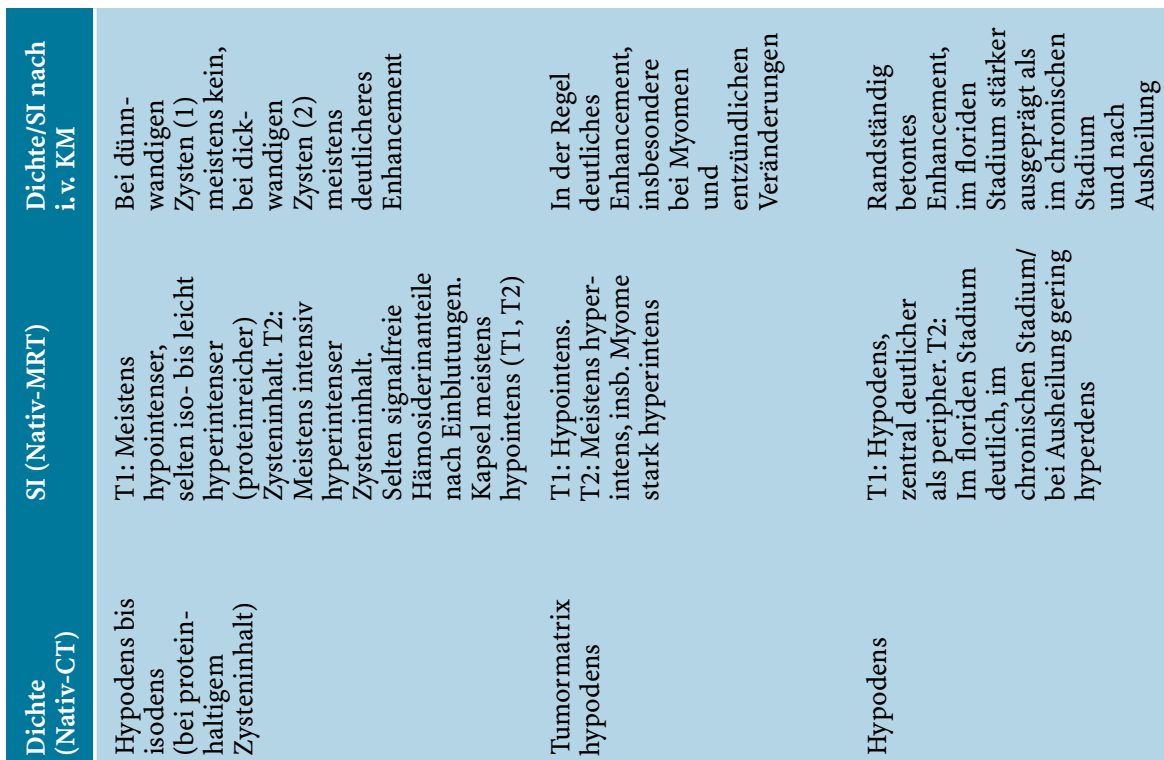

औ
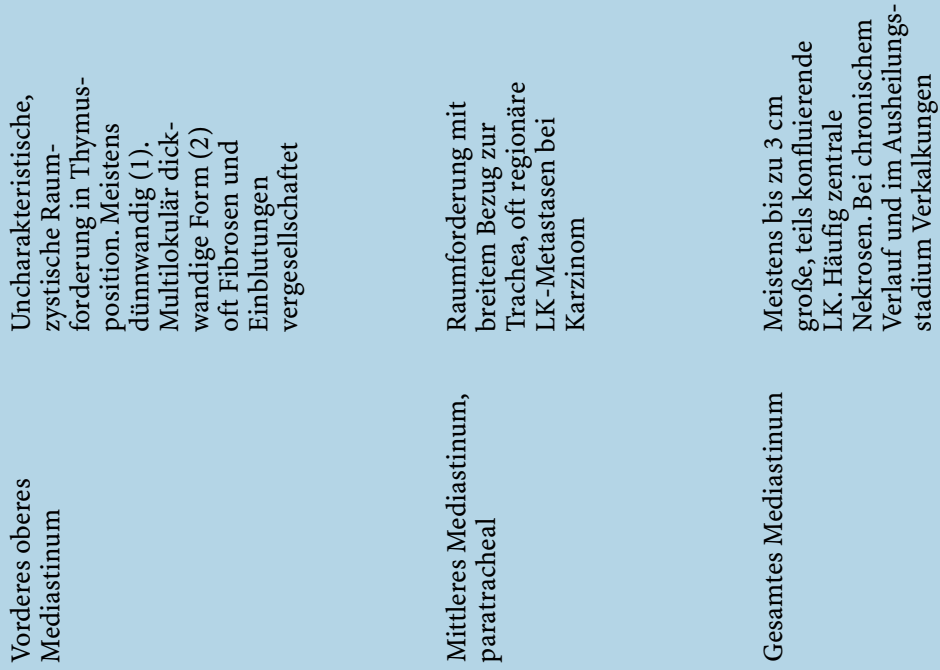

E్
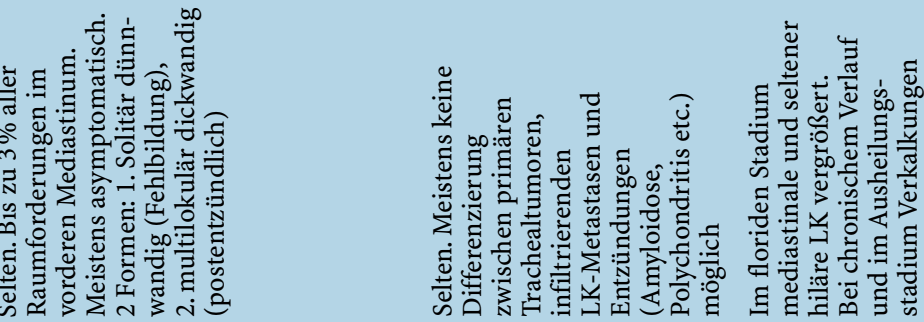

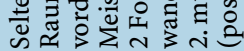

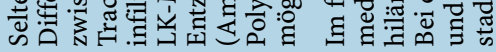
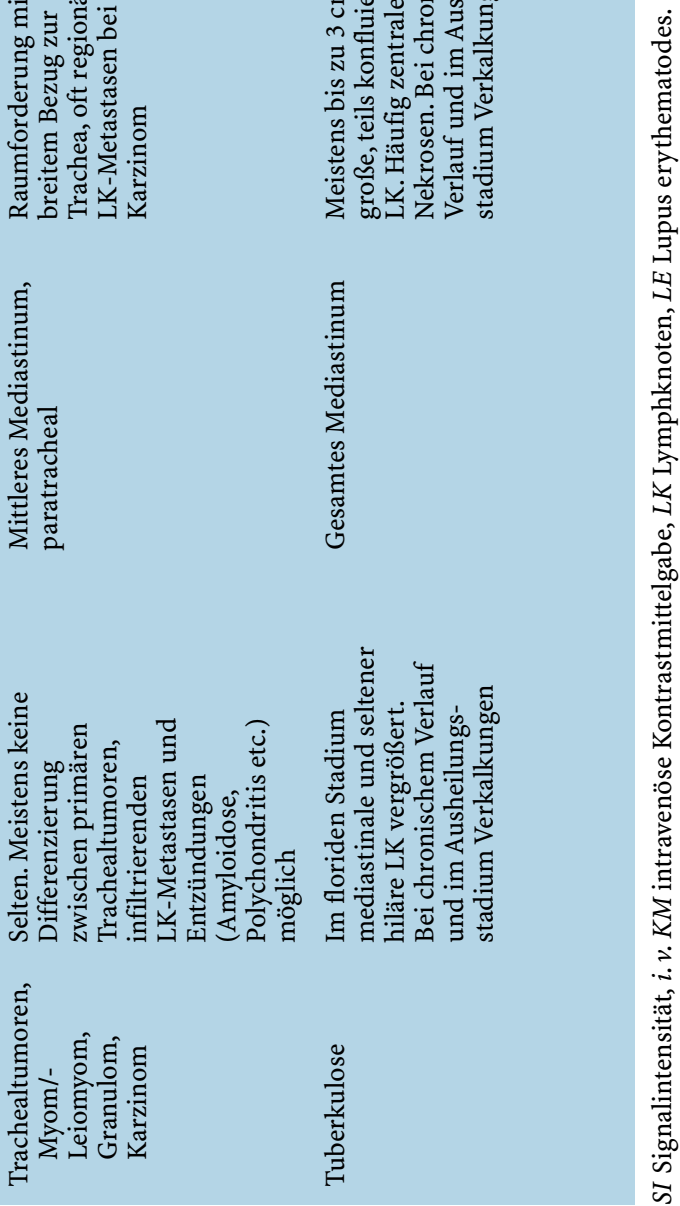


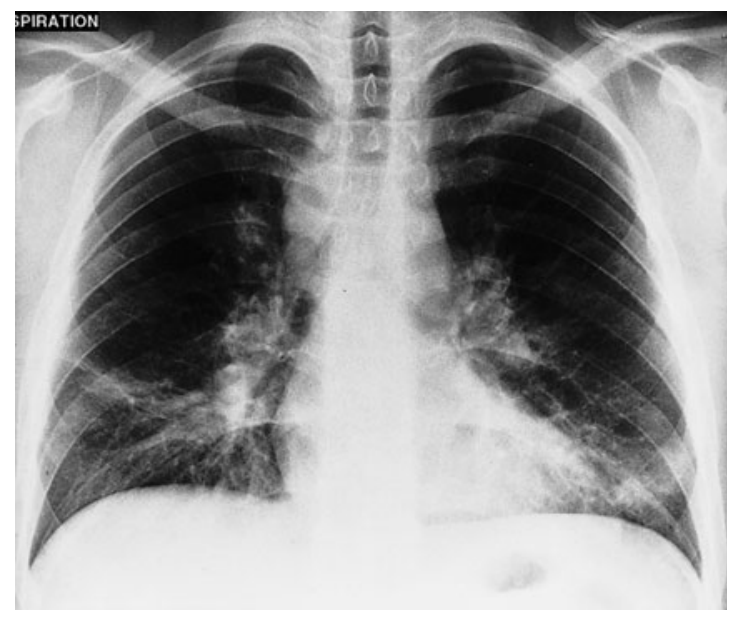

Abb. 17.36. Sarkoidose, Stadium II. Die sagittale Thoraxübersichtsaufnahme zeigt eine beidseitige Verbreiterung des oberen Mediastinums, seitensymmetrisch verbreiterte und verdichtete Hili sowie streifige pulmonale Verdichtungen

werden bevorzugt unilaterale, zumindest jedoch seitenasymmetrische Hilusverbreiterungen gefunden. Die sehr seltene angiofollikuläre Hyperplasie ist bevorzugt im mittleren und hinteren Mediastinum lokalisiert und führt zu einer solitären, glatt begrenzten lobulierten Verdichtung.

Die Sarkoidose entwickelt in bis zu $90 \%$ mediastinale und hiläre Raumforderungen, die im Gegensatz zur Tuberkulose seitensymmetrisch ausgebildet sind (Abb. 17.36). Ein mediastinaler Befall ohne hiläre Lymphknotenbeteiligung ist für die Sarkoidose untypisch. Bei Fehlen pulmonaler Veränderungen ist differentialdiagnostisch an ein malignes Lymphom $\mathrm{zu}$ denken, da die Lymphknotenvergrößerungen sowohl bei der Sarkoidose als auch bei malignen Lymphomen $\mathrm{zu}$ Konglomerattumoren konfluieren können.

Nodale Verkalkungen werden als Folge chronischer Entzündungen oder Ausheilungen einer Tuberkulose, Histoplasmose, Sarkoidose, Silikose oder Amyloidose beobachtet (Abb. $17.35 \mathrm{a}, \mathrm{b}$ ). Im Ausheilungsstadium einer Lymphknotentuberkulose weisen grobschollige oder homogene Verkalkungen auf die abgelaufene Entzündung hin. Grobschollige oder „eierschalenförmige“ Verkalkungen gelten als pathognomonisch für die Silikose. Nodale Verkalkungen sind untypisch für eine Asbestose und eine angiofollikuläre Hyperplasie.

\section{Schnittbilddiagnostik}

Die Aufgabe der Schnittbilddiagnostik liegt - bei entsprechender klinischer Relevanz - in der Darstellung kleinerer, projektionsradiographisch nicht erkennbarer Lymphknoten, in der topographischen $\mathrm{Zu}$ -
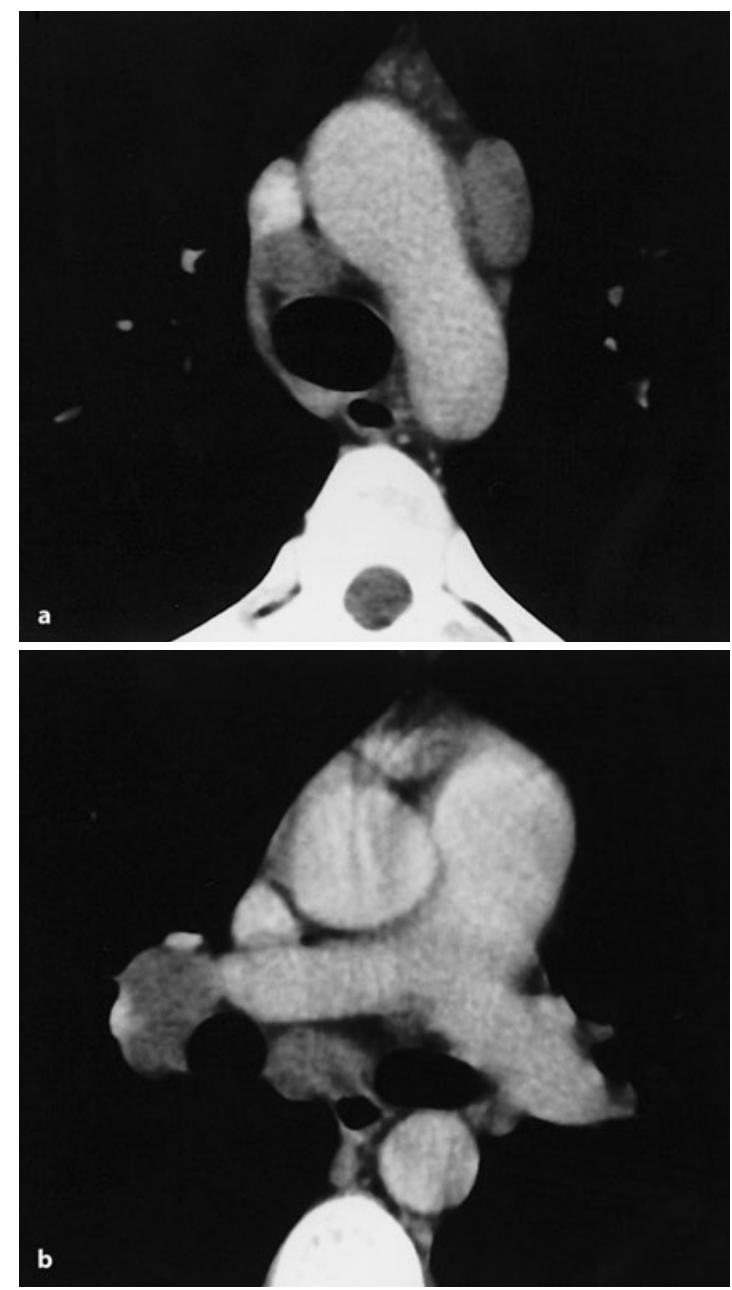

Abb. 17.37 a, b. Mediastinale (a, b) und hiläre (b) Lymphknotenvergrößerungen bei Sarkoidose. CT nach intravenöser Kontrastmittelgabe

ordnung und Ausdehnungsbestimmung vergrößerter Lymphknoten und im Nachweis bzw. Ausschluss pulmonaler Begleitbefunde (Abb. 17.37; Bittner 1998; Ecklund 1994; Gawne-Cain 1996; Kohman 1993; Lange 1996; Leppert 1998; Lesko 1999; Moon u. Han 1994; Moon 1996; Murray 1992; SchaeferProkop 1998; Siegel 1996; Wegener 1992; Wilson 1994). Den MR-tomographischen Vorteilen einer höheren Kontrastauflösung und einer multiplanaren Schichtwahl stehen die computertomographischen Vorteile einer höheren Ortsauflösung und einer überlegenen Abbildung von Verkalkungen gegenüber.

Es existieren keine CT- oder MRT-Kriterien zur Unterscheidung von Erkrankungen, die durch bakterielle, virale, mykoplasmatische, mykotische und parasitäre Erreger hervorgerufen werden. Bei bakte- 
riellen Pneumonien sind hiläre und mediastinale Lymphknotenvergrößerungen selten. Virus- und Pilzpneumonien hingegen weisen häufig bihiläre Lymphknotenvergrößerungen auf. Mykoplasmenund Pneumocystis-carinii-Infektionen unter Pentamidin-Prophylaxe führen typischerweise zu ausgeprägten mediastinalen Lymphknotenverkalkungen. Selten wird eine mediastinale Lymphadenopathie als Folge eines M. Crohn oder eines Soorbefalls der Speiseröhre beobachtet. Bei mediastinalen Lymphknotenvergößerungen infolge einer Thorakotomie sind Anamnese und Begleitbefunde wegweisend. Nach intravenöser Kontrastmittelgabe zeigen entzündlich veränderte Lymphknoten meistens ein homogenes Enhancement. Dies gilt insbesondere für Castleman-Tumoren und granulomatöse Lymphknotenerkrankungen. Zentrale Hypodensitäten als Ausdruck nekrotischer Einschmelzungen weisen auf Staphylokokken- und Tuberkuloseinfektionen hin.

Die überwiegend im mittleren und hinteren Mediastinum lokalisierte angiofollikuläre Lymphknotenhyperplasie stellt sich als solitärer, solider Tumor dar, der aufgrund seines Gefäßreichtums ein starkes Enhancement nach intravenöser Kontrastmittelgabe aufweist. Differentialdiagnostisch kommen hypervaskularisierte Metastasen (Schilddrüsenkarzinom, Nierenzellkarzinom), Hämangiome, intrathorakale Strumen und Nebenschilddrüsenadenome in Betracht.

Im floriden Stadium einer Tuberkulose können hiläre, paratracheale und tracheobronchiale Lymphknotenvergrößerungen nachzuweisen sein, die meistens asymmetrisch ausgebildet sind und typischerweise $\mathrm{zu}$ Konglomeraten konfluieren (Abb.17.23 c). Zentrale Hypodensitäten weisen auf nekrotische Einschmelzungen hin. Nach intravenöser Kontrastmittelgabe ist in bis zu $90 \%$ der Fälle ein nodales Randenhancement erkennbar, das sich von dem einer Lymphknotenmetastase eines Plattenepithelkarzinoms durch seine größere Breite $\geqq 20 \%$ des Lymphknotendurchmessers - unterscheidet. Diagnostisch wegweisend ist der Nachweis von tuberkulösen Lungeninfiltraten und Kavernen. Im chronischen Stadium einer Lymphknotentuberkulose finden sich nodale Verkalkungen, die bei unklaren pulmonalen Veränderungen den Verdacht auf eine alte Tuberkulose lenken (Gawne-Cain 1996).

Bei der Sarkoidose lassen sich paratracheal und präaortal im oberen Mediastinum sowie beidseits hilär vergrößerte und symmetrisch angeordnete Lymphknoten nachweisen (Abb. 17.37). Der symmetrische hiläre Befall grenzt die Sarkoidose von der Tuberkulose mit ihrem meist unilateralen, zumindest aber asymmetrischen Befallmuster ab. Bei vorwiegend mediastinalem Befall und fehlenden Lungenveränderungen ist differentialdiagnostisch an ein malignes Lymphom zu denken. Das nodale Densitätsverhalten vor und nach intravenöser Kontrastmittelgabe ergibt keine charakteristischen Hinweise. Im Abheilungsstadium werden grobschollige Verkalkungen beobachtet.

Bei der Silikose lassen sich meist einseitig vergrößerte mediastinale und hiläre Lymphknoten mit typischen grobscholligen Verkalkungen und uncharakteristischem Densitätsverhalten vor und nach intravenöser Kontrastmittelgabe nachweisen. Diagnostisch wegweisend sind Anamnese und pulmonaler Befund. Bei der differentialdiagnostisch in Betracht zu ziehenden Asbestose fehlen in der Regel nodale Verkalkungen.

Bei der Amyloidose findet sich in etwa $20 \%$ eine Beteiligung der mediastinalen und retroperitonealen paraaortalen Lymphknoten. Die Amyloidspeicherungen führen zu einer Volumenzunahme des Lymphknotenparenchyms, wobei gelegentlich gesprenkelte Verkalkungen beobachtet werden. Die Anamnese eines Plasmozytoms, einer rheumatoiden Arthritis, einer Tuberkulose oder eines familiären Mittelmeerfiebers weisen auf sekundäre Formen der Amyloidose hin.

\subsection{5}

\section{Pseudotumoren}

\section{Pathologie}

Unter Pseudotumoren werden umschriebene oder diffuse Mediastinalprozesse subsummiert, die projektionsradiographisch als Raumforderung eingeordnet werden können, aber nicht tumoröser Genese sind (Tabelle 17.1, 17.4; Hofmann 1991; Kohman 1993; Lackner 1998; Lange 1996; Merten 1991; Miller 1995; Padhani, Rodriguez 1995; Roger 1998; SchaeferProkop 1998; Siegel 1996; Sippel 1997; Wegener 1992).

\footnotetext{
Häufigste mediastinale Pseudotumoren

- Mediastinale Einblutungen

$\nabla$ Infolge von Verletzungen mediastinaler Venen und Arterien, nach versuchten Katheterplatzierungen in die V. cava superior (häufigste Ursache), bei Herz- und Gefäßoperationen, bei stumpfen oder perforierenden Thoraxtraumen, bei rupturierten Aortenaneurysmen (in $>90 \%$ distal des Abgangs der linken A. suclavia), bei rupturierten Aortendissektionen oder infolge einer Antikoagulantientherapie

- Arterielle Gefäßveränderungen

$\checkmark$ Wie degenerative, mykotische oder posttraumatische Aortenaneurysmen oder - seltener - Aneurysmen der A. pulmonalis, poststenotische Dilatationen und Verlaufsanomalien der größeren Gefäße

- Erweiterte Kollateralgefäße

$\checkmark$ Wie die V.azygos als kavokavale Anastomose, die A. thoracica interna bei Aortenisthmusstenose, erweiterte Venen bei akuter Thrombophlebitis oder bei Ösophagusvarizen
} 
- Primäre oder per continuitatem aus der Nachbarschaft fortgeleitete Mediastinalabszesse

- Ein Infusionsmediastinum infolge einer nicht erkannten Katheterfehlposition

- Ein Chylomediastinum infolge einer Verletzung des D. thoracicus

- Atelektasen oder pneumonische Infiltrate im linken Unterlappen

- Zwerchfellhernien

$\checkmark$ Mit Verlagerung unterschiedlicher abdomineller Organanteile nach intrathorakal (in $90 \%$ der Fälle axiale, paraösophageale oder kombinierte Hiatushernien; Larreyoder Morgagni-Hernie: intrathorakaler Prolaps von Leber, Omentum, Dünndarm und/oder Dickdarm bevorzugt rechts, aber auch links parakardial durch angeborene Lücken im Trigonum sternocostale; Bochdalek-Hernie: intrathorakaler Prolaps von Magen, Omentum, Dünndarm, Dickdarm, Milz, Niere und/oder Leber durch eine in $90 \%$ der Fälle linksseitige angeborene Lücke im Trigonum lumbocostale)

- Diffuse oder umschriebene mediastinale Fetteinlagerung ohne Krankheitswert als Normvariante bei Adipositas oder Kortisontherapie

- Eine extramedulläre Hämatopoese bei einer Thalassämie oder Sichelzellanämie mit paravertebral gelegenen Pseudotumoren

\section{Klinik}

Anamnese und Klinik weisen in vielen Fällen die diagnostische Richtung. Falls dies nicht der Fall ist, ist die Ätiologie mediastinaler Pseudotumoren mehrheitlich computer- oder MR-tomographisch zu klären. Bei entsprechendem Verdacht geben die gastrointestinale Hohlraumdiagnostik, die Angiographie oder die Phlebographie weitere differentialdiagnostische Hinweise.

\section{Projektionsradiographie}

Auf der Thoraxübersichtsaufnahme gelingt erst bei größeren Pseudotumoren der Nachweis einer umschriebenen oder diffusen Mediastinalverbreiterung (Kohman 1993; Lackner 1998; Lange 1996; Merten 1991; Miller 1995; Murray 1992; Sippel 1997). Begleitbefunde wie Pleuraergüsse, Lungenkontusionen, Rippenfrakturen, sternale Cerclagen, zentrale Venenkatheter oder spondylodiscitische Wirbelsäulenveränderungen engen bei entsprechender Klinik die Differentialdiagnose ein.

Als röntgenologisches Korrelat eines ausgedehnteren Mediastinalhämatoms findet sich eine Verbreiterung des oberen Mediastinums mit unscharfen Randkonturen (Abb. 17.38 a, d). Das Blut kann sich extrapleural bis über die Lungenspitzen ausbreiten. Vereinzelt findet sich ein linksseitiger Hämatothorax.

Dilatative Pathologien der thorakalen Aorta (Aneurysmen, poststenotische Dilatationen) lassen sich häufig aufgrund pathognomonischer Wandverkal- kungen dem Ursprungsgefäß zuordnen (Cole 1995; Ferreira 1997; Abb. 17.39 a, b). Verlaufsanomalien der größeren Gefäße oder Kollateralkreisläufe bedürfen bei therapeutischer Relevanz einer angiographischen oder phlebographischen Klärung (Padhani 1998).

Atelektasen oder Infiltrate insbesondere im linken Unterlappen können auf der sagittalen Thoraxübersichtsaufnahme zu einer umschriebenen paravertebralen Verdichtung führen (Abb. 17.40), die mit einem Tumor im hinteren Mediastinum verwechselt werden kann. Die klinischen Symptome und der Nachweis einer unscharfen Berandung des Pseudotumors zur Lungenperipherie weisen auf die Diagnose hin.

Bei Zwerchfellhernien variiert die Bildmorphologie mit dem Volumen und der Zusammensetzung der nach intrathorakal verlagerten Gewebeanteile und somit indirekt mit der Größe der Zwerchfelllücke (Abb. 17.41 a). Die dem Bruchsack entsprechende Verdichtung weist immer einen breiten Kontakt zum Zwerchfell auf. Enthält eine Morgagni-Hernie überwiegend Lebergewebe, so zeigt sich auf der Thoraxübersichtsaufnahme eine homogene, rechts parakardiale Verdichtung, die nicht von perikardialem Fett oder einer perikardialen Zyste zu differenzieren ist. Die bei stärkerer Ausprägung meist Kleinkinder betreffenden Bochdalek-Hernien sind in der Regel links laterodorsal, selten rechts laterodorsal im unteren Mediastinum lokalisiert. Luft innerhalb eines mediastinalen Pseudotumors wird beobachtet, wenn Magen- oder Darmanteile nach intrathorakal verlagert sind. Häufig finden sich in solchen Fällen pathognomonische zirkuläre oder semizirkuläre, Kerckring-Falten oder Haustren entsprechende Randbegrenzungen der intrathorakalen Lufteinschlüsse. Eine gastrointestinale Hohlraumdiagnostik liefert in solchen Fällen weiteren Aufschluss.

Pleuroperikardiale Fetteinlagerungen treten häufiger beidseitig auf. Sie sind jedoch meistens unter Bevorzugung der rechten Seite asymmetrisch ausgebildet.

\section{Schnittbilddiagnostik}

Computer- und MR-Tomographien ermöglichen es, die Qualität mediastinaler Flüssigkeitsansammlungen (Blut, Lymphe, Abszessinhalt) anhand ihrer Dichte bzw. Signalintensität einzuordnen und dilatative Gefäßveränderungen als Ursache vaskulärer Pseudotumoren nachzuweisen (Cole 1995; Kohman 1993; Ferreira 1997; Lange 1996; Lesko 1999; Merten 1991; Miller 1995; Murray 1992; Rodriguez 1995, Roger 1998; Schaefer-Prokop 1998; Siegel 1996; Sippel 1997; Wegener 1992; Wilson 1994).

Wird eine traumatische oder dissektionsbedingte Aortenruptur als Ursache eines Mediastinalhämatoms vermutet, so ist je nach klinischer Dringlichkeit die Durchführung einer Computertomographie oder 




Abb. 17.38 a-f.

Mediastinale Einblutungen.

a, b Iatrogene Verletzung der V. cava superior. Die sagittale Thoraxübersichtsaufnahme (a) zeigt eine glatt begrenzte Verbreiterung des oberen Mediastinums, das CT nach Anspritzen des zentralen Venen-

katheters (b) das hyperdense

Kontrastmittel extraluminal im

mediastinalen Fett-/Bindegewebe.

$\mathrm{c}-\mathrm{f}$ Iatrogene Aortenperforation.

Thoraxübersichtsaufnahme

vor (c) und nach Perforation (d) mit im Verlauf neu aufgetretener

Mediastinalverbreiterung.

Computertomographisch (e) und

MR-tomographisch (f) direkter

Nachweis der leicht hyperdensen

bzw. signalreichen mediastinalen Einblutung
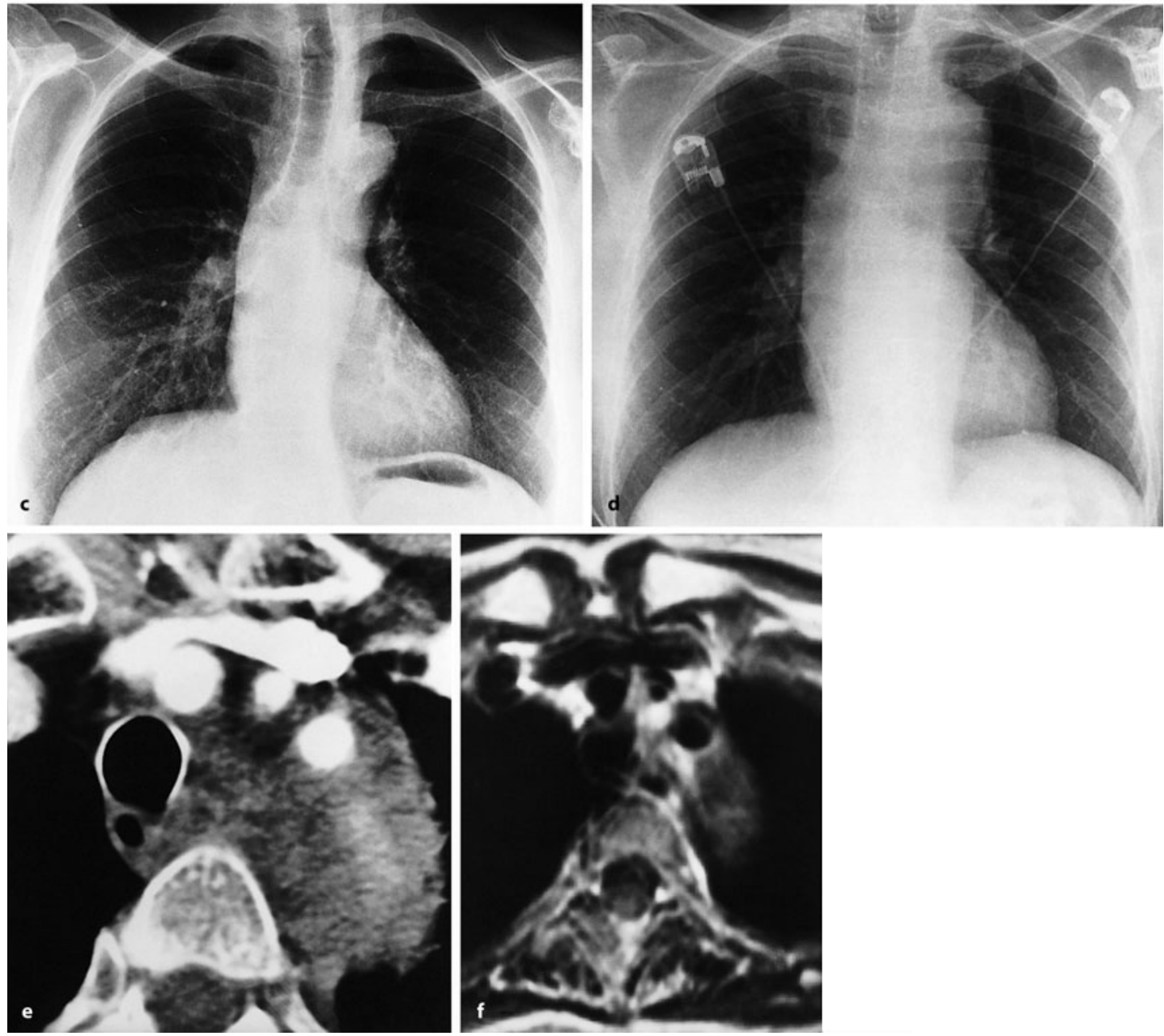

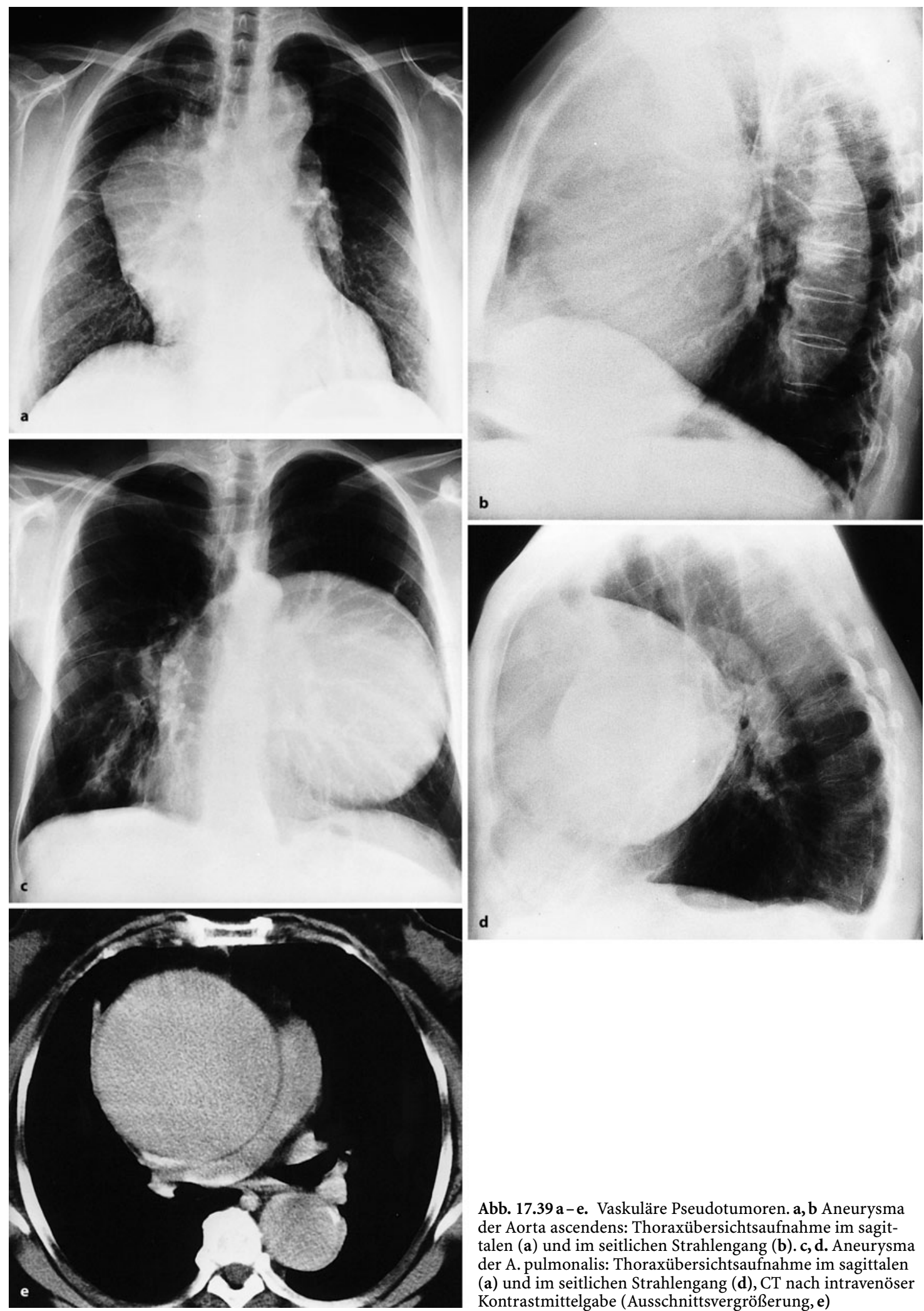

Abb. 17.39 a-e. Vaskuläre Pseudotumoren. a, b Aneurysma der Aorta ascendens: Thoraxübersichtsaufnahme im sagittalen (a) und im seitlichen Strahlengang (b). c, d. Aneurysma der A. pulmonalis: Thoraxübersichtsaufnahme im sagittalen (a) und im seitlichen Strahlengang (d), CT nach intravenöser Kontrastmittelgabe (Ausschnittsvergrößerung, e) 

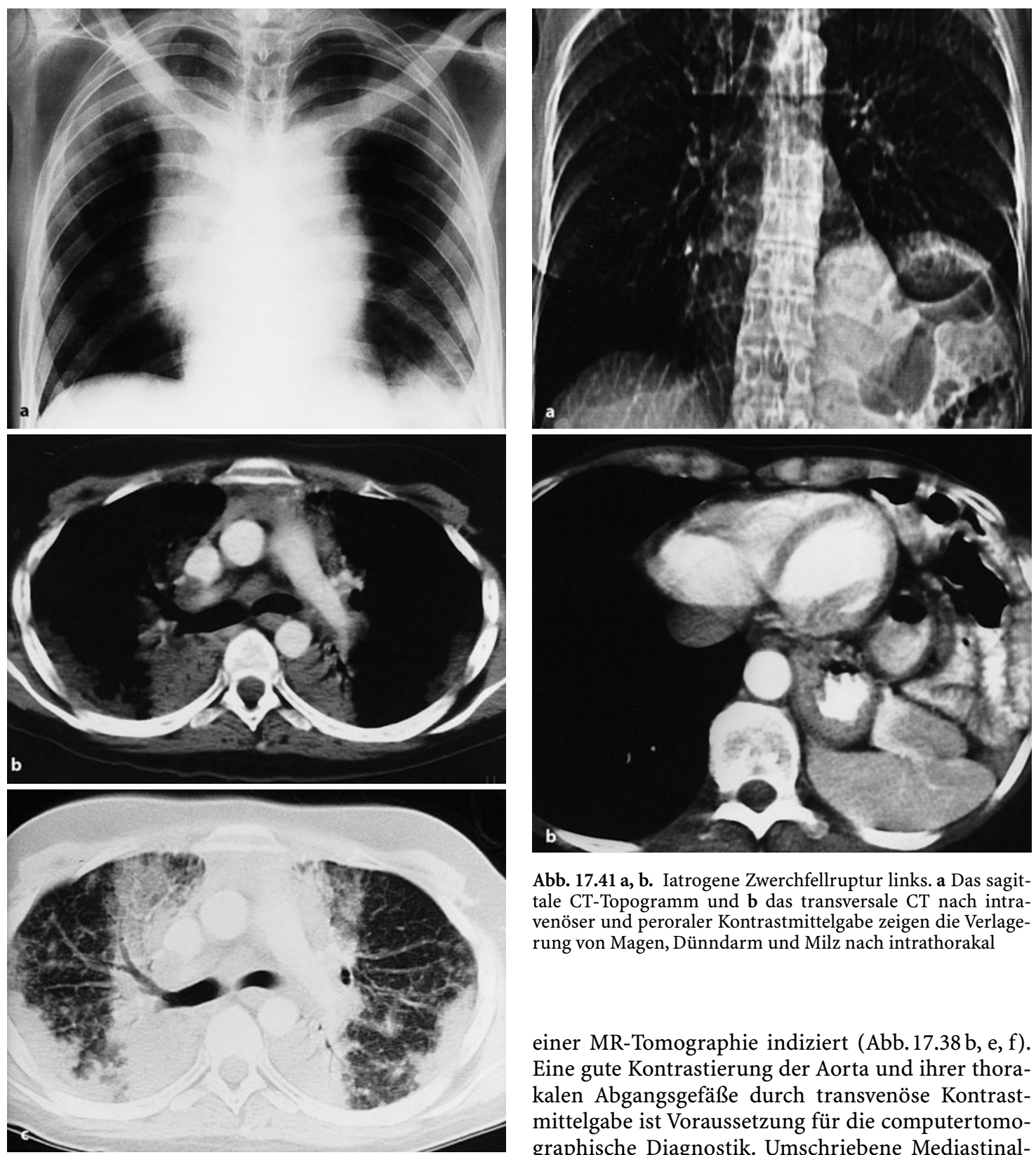

Abb. 17.40 a-c. Strahlenpneumonitis. a Sagittale Thoraxübersichtsaufnahme: Scheinbare Mediastinalverbreiterung. b CT mit Ausspielung im Mediastinalfenster: Reaktive mediastinale Lymphknotenvergrößerungen. c CT mit Ausspielung im Lungenfenster: Paramediastinale Infiltrate

Abb. 17.41 a, b. Iatrogene Zwerchfellruptur links. a Das sagittale CT-Topogramm und $\mathbf{b}$ das transversale CT nach intravenöser und peroraler Kontrastmittelgabe zeigen die Verlagerung von Magen, Dünndarm und Milz nach intrathorakal

einer MR-Tomographie indiziert (Abb.17.38 b, e, f). Eine gute Kontrastierung der Aorta und ihrer thorakalen Abgangsgefäße durch transvenöse Kontrastmittelgabe ist Voraussetzung für die computertomographische Diagnostik. Umschriebene Mediastinaleinblutungen stellen sich als Raumforderungen dar, die die anatomischen Leitstrukturen Trachea, Ösophagus und große Mediastinalgefäße in wechselndem Ausmaß verdrängen. Diffuse Einblutungen obliterieren die physiologischen mediastinalen Fettgewebsschichten. Die Dichte bzw. Signalintensität eines Hämatoms hängt von seinem Alter ab. Durch das fehlende Enhancement nach transvenöser Kontrastmittelgabe lässt sich ein scharf berandetes, umschriebenes Hämatom von Neoplasien abgrenzen.

Mediastinale Abszesse unterscheiden sich bildmorphologisch nicht von Abszessen in anderen Kör- 


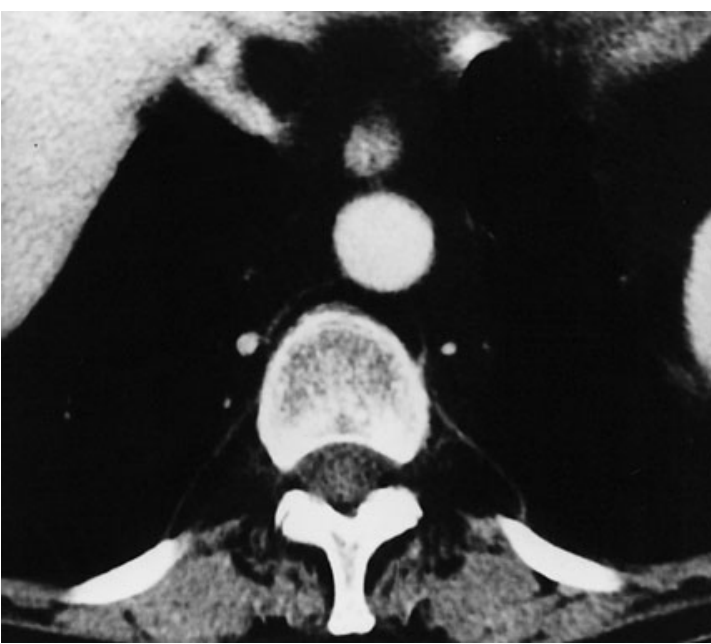

Abb. 17.42. Mediastinale Lipomatose. Ausschnittsvergrößerung eines Computertomogramms mit transvenöser Kontrastierung der V. azygos und der V. hemiazygos

perregionen. Ihr liquider Inhalt grenzt sich computertomographisch hypodens gegenüber dem entzündlichen Granulationsgewebe im Randbereich ab. Aufgrund der Hypervaskularisation des Granulationsgewebes kommt es zu einem starken Enhancement nach intravenöser Kontrastmittelgabe. MR-tomographisch stellt sich die zentrale Kolliquationsnekrose in T1-Wichtung signalarm und in T2-Wichtung signalreich dar. Ähnlich wie in der Computertomographie demarkiert sich das periphere Granulationsgewebe am besten auf T1-gewichteten Bildern nach intravenöser Kontrastmittelgabe.

Bei Hernien sind die nach mediastinal bzw. intrathorakal verlagerten Organstrukturen (Magen, Dünndarm, Dickdarm, Omentum, Leber, Milz, Nieren) oberhalb des Zwerchfells nachzuweisen (Abb. 17.41b). Prolabierte Anteile des Gastrointestinaltraktes sind durch perorale Kontrastierung des Magen-DarmTraktes leicht zu erkennen. Bei Hiatushernien weisen die Zwerchfellschenkel eine Dehiszenz von mehr als $15 \mathrm{~mm}$ auf. Fett innerhalb des Bruchsackes ist auf prolabierte Omentumanteile zurückzuführen.

Pleuroperikardiale Fetteinlagerungen und mediastinale Lipomatosen sind an ihren fettäquivalenten Dichten bzw. Signalintensitäten zu diagnostizieren (Rodriguez 1995). Die mediastinale Lipomatose zeichnet sich durch eine im oberen Mediastinum betonte diffuse Vermehrung des mediastinalen Fettgewebes aus (Abb. 17.42). Inhomogenitäten weisen auf Fibrosen, Einblutungen, akute oder chronische Entzündungen, Tumorinfiltrationen oder Operationsbzw. Bestrahlungsfolgen als Ursache des vermehrten Fettgewebsanteils hin.
Eine extramedulläre Hämatopoese kann zu polyzyklisch begrenzten, soliden paravertebralen Pseudotumoren im unteren Mediastinum führen (Roger 1998). Im Gegensatz zu neurogenen Tumoren liegen keine Knochenarrosionen vor. Eine Splenomegalie und Zeichen einer Knochenmarkshyperplasie sind pathognomonische Begleitbefunde.

\section{5}

\section{Sonstige Erkrankungen}

\subsection{1 \\ Mediastinalemphysem}

\section{Pathologie und Klinik}

Mediastinale Lufteinschlüsse, die sich entlang des Perikards, des Ösophagus, der Trachea und der großen Gefäße ausbreiten, werden unter dem Begriff "Mediastinalemphysem“ oder „Pneumomediastinum “ zusammengefasst (Lackner 1998; Lange 1996; Wegener 1992). Luft kann auf unterschiedlichen Wegen in das Mediastinum gelangen. Meist ist das Mediastinalemphysem Folge einer alveolären Ruptur, die durch einen starken intraalveolären Druckanstieg bei künstlicher Beatmung mit positivem endexspiratorischen Druck („positive endexpiratory pressure“, PEEP), tiefen Atemexkursionen, Husten, Valsalvamanöver, schwerem Erbrechen, stumpfem Thoraxtrauma und plötzlichem Abfall des atmosphärischen Druckes (Barotrauma bei Tauchern und Piloten) hervorgerufen wird. Über Alveolareinrisse gelangt Luft entlang der peribronchialen Spalträume in das mediastinale Interstitium. Auf ähnlichem Wege kann ein Pneumothorax zu einem Mediastinalemphysem führen. Selten sind Ösophagus- oder Tracheobronchialrupturen Ursache eines Mediastinalemphysems. Nach zervikalen Traumen und Zahnextraktionen kann Luft von kranial in das Mediastinum eintreten. Über präformierte Zwerchfelllücken ist ein Übertritt von Luft aus dem Abdomen in das Mediastinum möglich. Klinisch bestehen häufig begleitende zervikale und thorakale Weichteilemphyseme, seltener eine obere Einflussstauung und eine Atembehinderung.

\section{Projektionsradiographie}

Auf der Thoraxübersichtsaufnahme zeigen sich als Ausdruck eines Mediastinalemphysems vertikale streifige Lufteinschlüsse in Projektion auf das Mediastinum, die den vorgegeben anatomischen Leitstrukturen folgen (Abb.17.43a, b; Lackner 1998; Lange 1996; Murray 1992). Die mediastinale Pleura ist typischerweise lateralisiert und durch eine Luftschicht vom mediastinalen Fett-/Bindegewebe abgehoben. Hierdurch kann die linke Herzkontur deutlicher als 


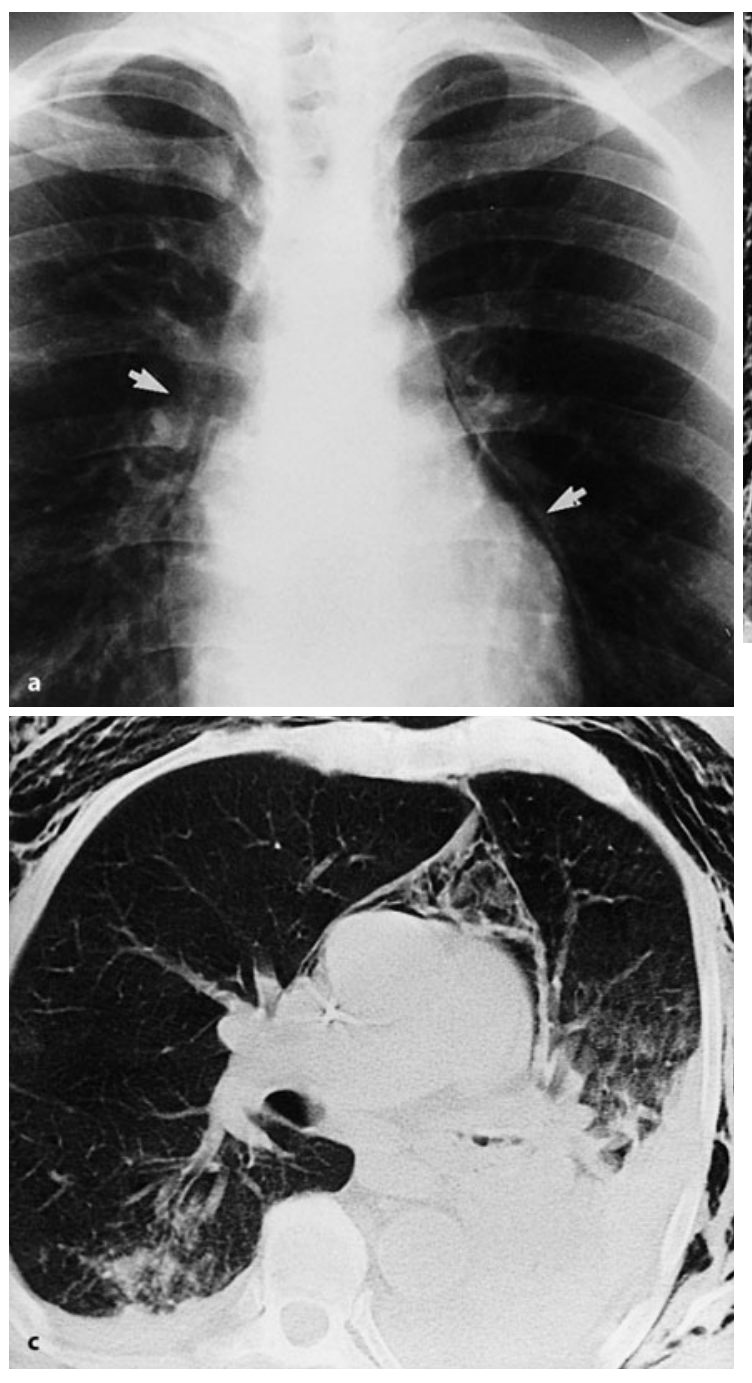

Abb. 17.43 a-c. Mediastinalemphysem.

Mediastinale Lufteinschlüsse auf der sagittalen Thoraxüber- unter Normalbedingungen abzugrenzen sein. Gelegentlich - vor allem beim ,adult respiratory distress syndrome" (ARDS) - ist ein über die Hili mit den mediastinalen Lufteinschlüssen kommunizierendes Lungenemphysem nachzuweisen. Ein häufiger links lokalisierter Seropneumothorax kann Folge einer Ösophagusperforation sein.

\section{Schnittbilddiagnostik}

Die Computertomographie ermöglicht als Schichtbildverfahren eine überlagerungsfreie und damit eine frühere und anatomisch-topographisch präzisere Darstellung der mediastinalen Luftverteilung als die Thoraxübersichtsaufnahme (Abb.17.43c; Lackner 1998; Lesko 1999; Murray 1992; Schaefer-Prokop 1998; Wegener 1992; Wilson 1994). Weist die Klinik auf ein entzündliches Geschehen hin, so ist eine Insichtsaufnahme (Pfeil) (a) bei einem Patienten mit Ösophagusperforation sowie auf dem CT-Topogramm (b) und dem im Lungenfenster ausgespielten CT (c) eines anderen Patienten mit Rippenfrakturen und Thoraxwandemphysem

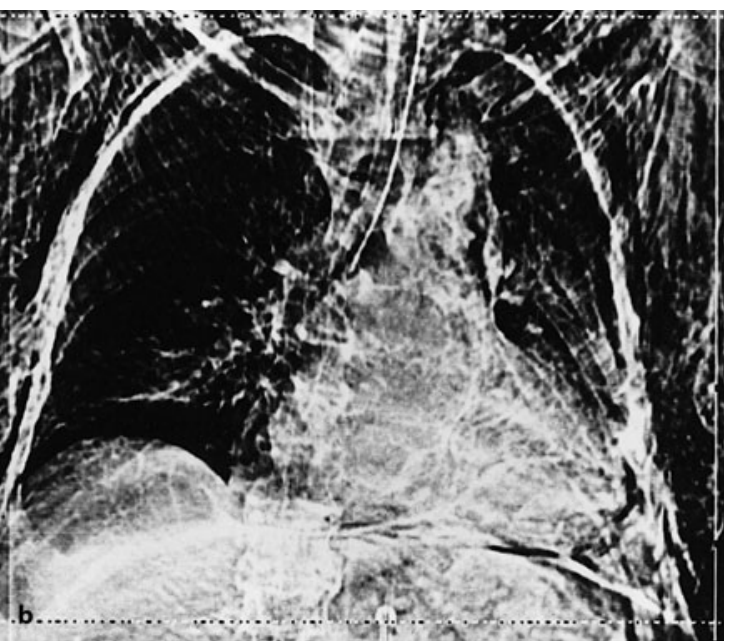

fektion durch gasproduzierende Bakterien differentialdiagnostisch in Betracht zu ziehen. In der Regel gelingt aber auch computertomographisch keine Klärung der Ursache eines Mediastinalemphysems.

\subsection{2 \\ Mediastinalverlagerungen}

\section{Pathologie und Klinik}

Pleurale und pulmonale Volumenänderungen können zu Verlagerungen des Mediastinums führen (Lackner 1998; Lange 1996; Wegener 1992). Mediastinalverlagerungen sind Folge eines einseitigen intrathorakalen Unter- oder Überdrucks. Sie können fixiert oder respiratorischen Schwankungen unterworfen sein. Unter pathophysiologischen Gesichts- 
punkten werden permanente statische Verlagerungen, vorübergehende statische Verlagerungen und dynamische Verlagerungen unterschieden.

Permanente statische Mediastinalverlagerungen können durch Pneumektomien, schrumpfende Pleuraschwarten, pulmonale oder pleurale Tumoren oder Deformierungen des Thoraxskeletts verursacht sein. Vorübergehende statische Verlagerungen werden bei Atelektasen, größeren Pleuraergüssen und Pneumothoraces beobachtet. Bei beidseitiger thorakaler Pathologie können sich die Volumenänderungen gegenseitig aufheben, sodass das Mediastinum mittelständig bleibt.

Bei dynamischen Mediastinalverlagerungen pendelt das Mediastinum in Abhängigkeit von den intrathorakalen Druckverhältnissen (Atemexkursionen) hin und her. Ursächlich können ein Spannungspneumothorax, ein Thoraxwanddefekt mit Pleurafistel, eine Lungenüberblähung durch ein obstruktives Emphysem oder die Ventilfunktion eines aspirierten Fremdkörpers und eine einseitige Zwerchfellparese sein.

\section{Projektionsradiographie}

Als anatomische Fixpunkte zur Beurteilung der mediastinalen Mittelständigkeit dienen bei einer korrekten Bildeinstellung die im Normalfall rechts paramedian gelegene Trachea, der links paramedian gelegene Aortenbogen und die ca. 1-2 cm lateral der Wirbelsäule gelegene rechte Herzkontur (Lackner 1998; Lange 1996; Murray 1992). Hierbei sind die Inspirationstiefe sowie Herzvergrößerungen, Pleuraverdichtungen, pulmonale Infiltrate, Atelektasen und Wirbelsäulenskoliosen in die Analyse einzubeziehen.

Bei einer Verlagerung des gesamten Mediastinums einschließlich des Herzens sind beide Lungen unterschiedlich groß dargestellt (Abb. 17.44 a, 17.45). Werden nur Teile des Mediastinums verlagert, so kann sich dies an einer einseitigen Ausbuchtung einer Pleuraumschlagsfalte (Lungenhernie) zeigen. Pleurale, pulmonale und ossäre Begleitbefunde erlauben in den meisten Fällen Rückschlüsse auf die Ätiologie der Mediastinalverlagerung.

Abb. 17.44a-c. Mediastinalverlagerungen. a Zentrales Bronchialkarzinom rechts: Die sagittale Thoraxübersichtsaufnahme zeigt eine Mediastinalverlagerung nach ipsilateral aufgrund der Volumenminderung der rechten Lunge durch die obstruktionsbedingte Minderbelüftung. b und c Pneumektomie links mit ipsilateraler Verlagerung des Mediastinums auf der sagittalen Thoraxübersichtsaufnahme (b) und im CT (c)
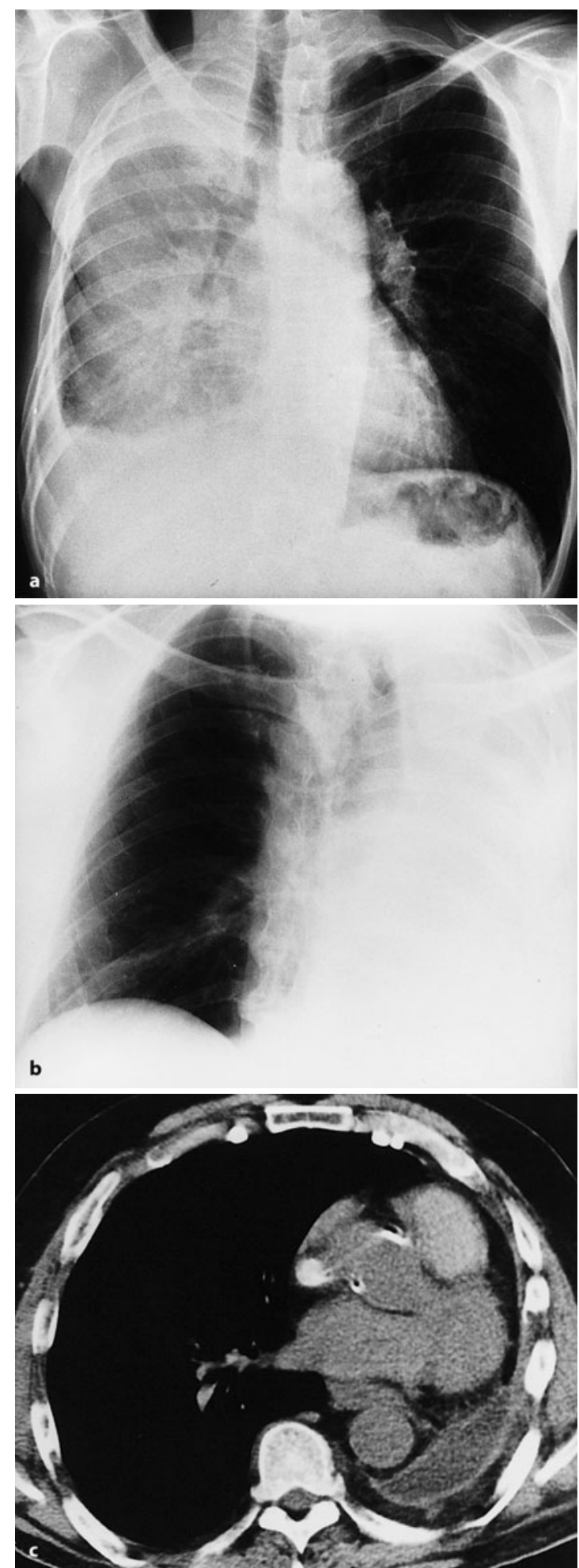

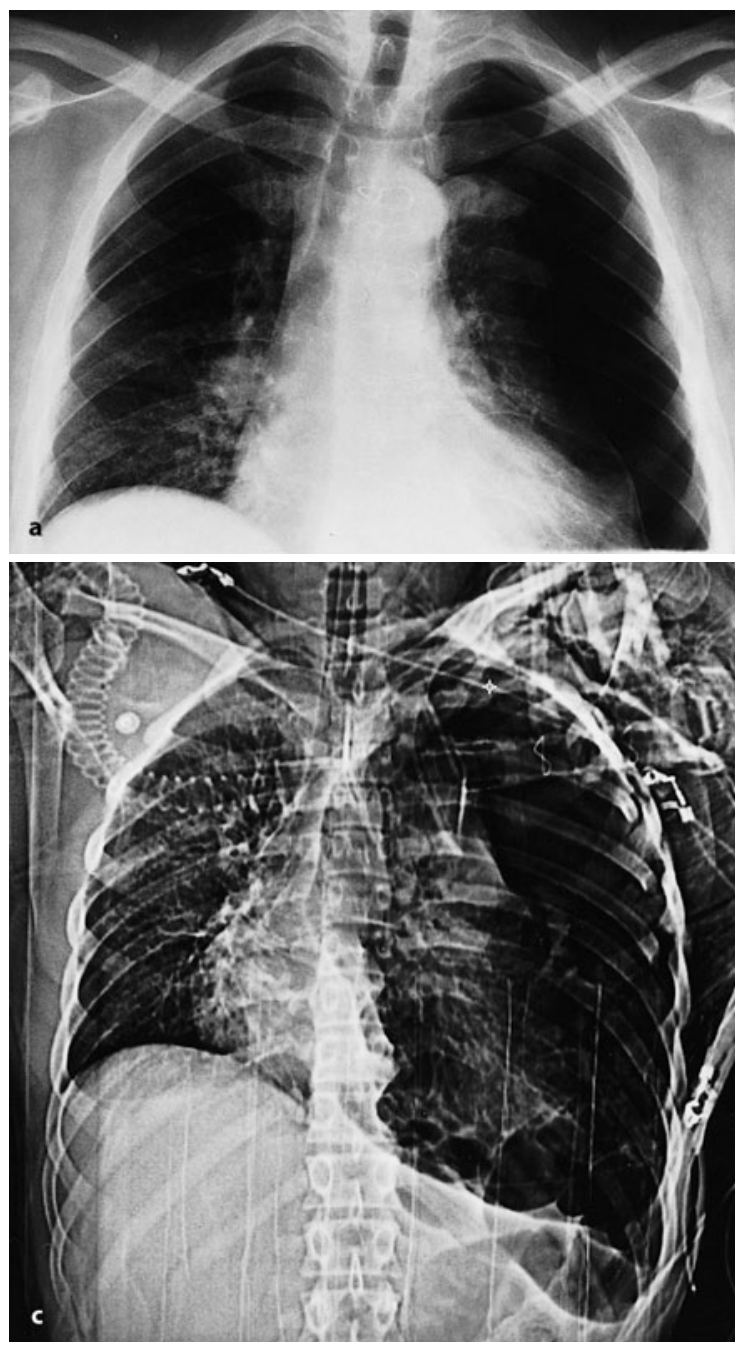

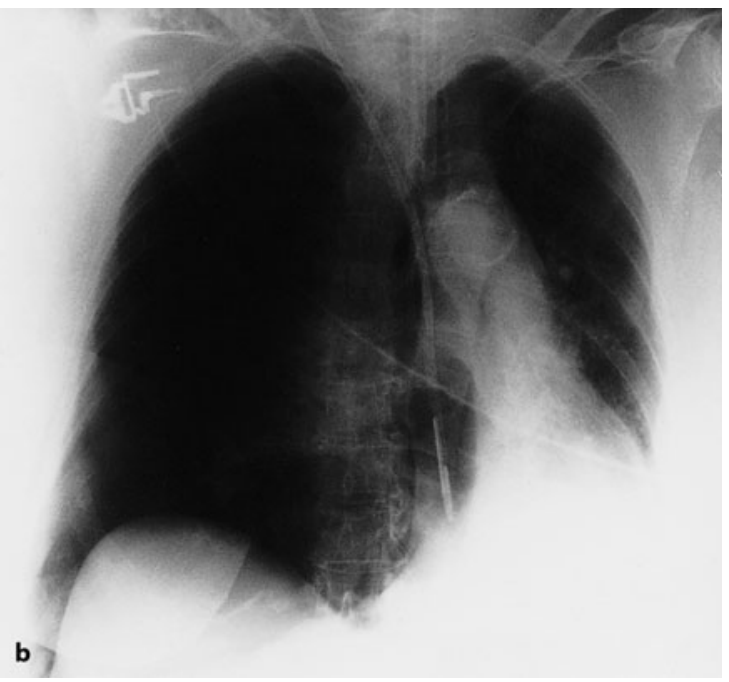

Abb. 17.45 a-c. Spannungspneumothoraces. a Sagittale Thoraxübersichtsaufnahme: Leichte Mediastinalverlagerung nach rechts aufgrund eines linksseitigen Spannungspneumothorax mit Ergusskomponente. Zustand nach ACVBOperation. b Sagittale Thoraxübersichtsaufnahme: Ausgeprägte Mediastinalverlagerung nach links aufgrund eines rechtsseitigen Spannungspneumothorax bei einem polytraumatisierten Patienten.c CT-Topogramm eines weiteren Patienten mit linksseitiger Rippenserienfraktur und Spannungspneumothorax: Mediastinalverlagerung nach rechts
Bei einer inspiratorischen Ventilstenose verlagert sich das Mediastinum inspiratorisch zur erkrankten Seite, da diese ihr Volumen nur mit Verzögerung erhöht. Bei einer exspiratorischen Ventilstenose wird das Mediastinum in Exspiration zur gesunden Seite verlagert, da diese ihr Volumen rascher reduziert als die Seite mit der exspiratorischen Bronchusstenose. Daher normalisiert sich die Mediastinalposition bei inspiratorischen Ventilstenosen in Exspiration und bei exspiratorischen Ventilstenosen in Inspiration. Diese dynamischen Vorgänge sind leicht auf Thoraxübersichtsaufnahmen in Inspiration und Exspiration zu erkennen. Auf eine exakte Ausführung der Atemanweisungen ist zu achten: $\mathrm{Da}$ eine Röntgenaufnahme nur den Zustand der Thoraxorgane während des
Bruchteils eines Sekunde wiedergibt, besteht die Möglichkeit, dass die Exposition in mediastinaler Mittelstellung erfolgt, sodass eine endinspiratorische oder endexspiratorische Mediastinalverlagerung dem Nachweis entgeht.

\section{Schnittbilddiagnostik}

Mediastinalverlagerungen werden projektionsradiographisch diagnostiziert. Der Einsatz von Schichtbildverfahren liefert hinsichtlich der Diagnose einer Mediastinalverlagerung keine Zusatzinformationen, kann jedoch bei der Zuordnung und prätherapeutischen Beurteilung der ursächlichen Pathologien wesentliche Hilfestellungen liefern (Tabelle 17.9, Abb. 17.44c). 


\section{Literatur}

Aktogu A et al. (1996) Bronchogenic cysts: Clinicopathologic presentation and treatment. Eur Respir J 9: 2017-2021

Allen HA et al. (1983) Leiomyoma of the trachea. Am J Roentgenol 141: 1015-1019

Anayanwu E (1991) Sonstige primäre gutartige und bösartige Mediastinaltumoren. In: Drings P, Vogt-Moykopf I (Hrsg) Thoraxtumoren. Diagnostik - Staging - gegenwärtiges Therapiekonzept. Springer, Berlin Heidelberg New York Tokyo, S 342-358

Anayanwu E, Krysa S (1991) Diagnostik der Mediastinaltumoren. In: Drings P, Vogt-Moykopf I (Hrsg) Thoraxtumoren. Diagnostik - Staging - gegenwärtiges Therapiekonzept. Springer, Berlin Heidelberg New York Tokyo, S 317-329

Astrom KG et al. (1996) CT-guided transsternal core biopsy of anterior mediastinal masses. Radiology 199: 564-567

Baran R et al. (1996) Intrathoracic tuberculous lymphadenopathy: Clinical and bronchoscopic features in 17 adults without parenchymal lesions. Thorax 51: 967-968

Belfiore G et al. (1997) Middle mediastinum lesions: Preliminary experience with CT-guided fine-needle aspiration biopsy with a suprasternal approach. Radiology 202: 870-873

Benzarti M et al. (1997) Primary calcified hydatid cyst producing compression. Rev Mal Respir 144: 53-54

Betsch B et al. (1994) Comparison of computerized tomography and B-image ultrasound in imaging diagnosis of the mediastinum. Bildgebung 61: 295-298

Beyer D, Neufang KFR (1980) Diagnostische Wertigkeit pleuromediastinaler Linien für Röntgenuntersuchungen des $\mathrm{Me}$ diastinums, Teil 2, Röntgenblätter 33: 335-347

Bittner RC, Felix R (1998) Magnetic resonance (MR) imaging of the chest: State-of-the-art. Eur Resp J 11: 1392-1404

Bonomo L et al. (1996) Lung cancer staging: The role of computed tomography and magnetic resonance imaging. Eur J Radiol 23: 35-45

Boyce GA (1994) Endoscopic evaluation of the patient with esophageal carcinoma. Chest Surg Clin N Am 2: 257-308

Brown RB, Aughenbaugh GL (1991) Masses of the anterior mediastinum: CT and MR imaging. Am J Roentgenol 157: $1171-1180$

Cascade PN et al. (1998) Variability in the detection of enlarged mediastinal lymph nodes in staging lung cancer: A comparison of contrast-enhanced and unenhanced CT. Am J Roentgenol 170: 927-931

Cesani F et al. (1995) Mediastinal scintigraphy for parathyroid localization in hyperparathyroidism. Am Surg 61: 796-798

Chang AY (1994) Neoplasms of the mediastinum, chest wall, and pleura. Cur Opin Radiol 6: 171-176

Chukwuemeka A et al. (1997) CT anatomy of the mediastinal structures at the level of the manubriosternal angle. Clin Anat 10: 405-408

Cifti E et al. (1998) MR imaging in a desmoid tumor of the posterior mediastinum with extension into the abdominal cavity. A case report. Acta Radiol 39: 301-303

Cole TJ et al. (1995) Normal and abnormal vascular structures that simulate neoplasms on chest radiographs: Clues to the diagnosis. Radiographics 15: 867-891

Cyna-Gorse F et al. (1996) Cervico-mediastinal recurrences of differentiated thyroid cancers: Value of MRI. J Radiol 77: 1195-2000

Daldrup $\mathrm{H}$ et al. (1998) MR imaging of thoracic tumors in pediatric patients. Am J Roentgenol 170: 1639-1644

Ecklund K, Hartnell GG (1994) Mediastinal Castleman disease: MR and MRA features. J Thorac Imaging 9: 156-159

Ferguson 1998; ER et al. (1998) Evaluation of complex mediastinal masses by magnetic resonance imaging. J Cardiovasc Surg 39: 117-119
Ferreira AC et al. (1997) Saphenous vein graft aneurysm presenting as a large mediastinal mass compressing the right atrium. Am J Cardiol 79: 706-707

Freitas JE, Freitas AE (1994) Thyroid and parathyroid imaging. Semin Nucl Medicine 24: 234-245

Gawne-Cain ML, Hansell DM (1996) The pattern and distribution of calcified mediastinal lymph nodes in sarcoidosis and tuberculosis: A CT study. Clin Radiol 51:363-367

Giron J et al. (1996) Diagnostic percutaneous thoracic punctures. Assessment through a critical study of a complication of 2406 cases. Rev Mal Respir 13: 583-590

Guyatt GH et al. (1995) Interobserver variation in the computed tomographic evaluation of mediastinal lymph node size in patients with potentially resectable lung cancer. Canadian Lung Oncology Group. Chest 107: 116-119

Haddon MJ, Bowen A (1991) Bronchopulmonary and neuroenteric forms of foregut anomalies. Imaging for Diagnosis and Management. Radiol Clin N Am 29: 214-254

Hainaut P et al. (1998) Tuberculous mediastinal lymphadenopathy. Acta Clin Belg 53: 114-116

Hiddemann W et al. (1996) Lymphoma classification - the gap between biology and clinical management is closing. Blood 88: 4085-4089

Higgins CB (1993) Role of magnetic resonance imaging in hyperparathyreoidism. Radiol Clin N Am 31: 10171028

Hofmann WJ, Otto HF (1991) Die pathologische Anatomie der Mediastinaltumoren. In: Drings P, Vogt-Moykopf I (Hrsg) Thoraxtumoren. Diagnostik - Staging - gegenwärtiges Therapiekonzept. Springer, Berlin Heidelberg New York Tokyo, S 307-316

Kawashima A et al. (1992) CT and MR evaluation of posterior mediastinal masses. Cri Rev Diagn Imaging 33: 311-367

Ko SF et al.(1998) Mediastinal lipoblastoma with intraspinal extension: MRI demonstration. Magn Reson Imaging 16: 445-448

Kohman LJ (1993) Approach to the diagnosis and staging of mediastinal masses. Chest 103: 328S-330 S

Kushihashi $\mathrm{T}$ et al. (1996) Magnetic resonance imaging of thymic epithelial tumors. Crit Rev Diagn Imaging 37: 191-259

Lackner K-J (1998) Mediastinum. In: Thurn P, Bücheler E, Lackner KJ, Thelen $M$ (Hrsg) Einführung in die radiologische Diagnostik. Thieme, Stuttgart New York, S 316328

Lange S (1996) Radiologische Diagnostik der Thoraxerkrankungen. Thieme, Stuttgart New York

Leppert A (1998) Lymphknoten. In: Galanski M, Prokop M (Hrsg) Ganzkörper-Computertomographie. Thieme, Stuttgart New York, S 415-418

Lesko NM, Link KM (1999) Mediastinum and lung. In: Stark DD, Bradley WG jr (eds) Magnetic resonance imaging. Vol I. Mosby, St. Louis, S 355-371

Levasseur P et al. (1976) Profil des tumeurs chirurgicales du médiastin. A propos d'une série de 742 opérés. Nouv Presse Med 42: 2857-2859

Manegold C (1991) Maligne Lymphome des Mediastinums. Spezielle diagnostische und therapeutische Probleme der großen Lymphadenopathie. In: Drings P, Vogt-Moykopf I (Hrsg) Thoraxtumoren. Diagnostik - Staging - gegenwärtiges Therapiekonzept. Springer, Berlin Heidelberg New York Tokyo, S 359-365

Masaoka et al. (1981) Follow-up study of thymomas with special reference to their clinical stages. Cancer 48 : 2485-2492

Merten DF (1991) Diagnostic imaging of mediastinal masses in children. Am J Roentgenol 158: 825-832

Meschan I, Bechtold (1991) Mediastinale und hiläre Normabweichungen. In: Meschan I (Hrsg) Diagnostik mit bildgebenden Verfahren. Enke, Stuttgart, S 679-795 
Meusers P, Brittinger G (1997) R.E.A.L.-Klassifikation der Non-Hodgkin-Lymphome aus klinisch-onkologischer Sicht. Klin Onkol 97: 60-67

Miller WT (1995) Pleural and mediastinal disorders related to drug use. Semin Roentgenol 30: 35-48

Moon WK et al. (1996) Mediastinal tuberculous lymphadenitis: MR imaging appearance with clinicopathologic correlation. Am J Roentgenol 166: 21-25

Moon WK, Han MC (1994) Castleman's disease of the mediastinum: MR imaging features. Clin Radiol 49: 466-468

Murray JG et al. (1992) Imaging of the mediastinum and hili. Curr Opin Radiol 4: 44-52

Neufang KFR, Beyer D (1980) Diagnostische Wertigkeit pleuromediastinaler Linien für die Röntennativuntersuchung des Mediastinums, Teil 1. Röntgenblätter 33: 557-266

Neufang KFR, Bulo W (1981) Häufigkeit pleuromediastinaler Linien beim Gesunden. Ein Beitrag zur Kenntnis der normalen Röntgenanatomie der Mediastinalgrenzen. Fortschr Röntgenstr 135: 673-681

Neumann DP et al. (1998) Magnetic resonance imaging of a mediastinal ependymoma. Conn Med 62: 527-530

Nwose P et al. (1998) Mediastinal paraganglioma: A case report. Thorac Cardiovasc Surg 46: 376-379

O'Donovan PB (1994) The radiographic evaluation of the patient with esophageal carcinoma. Chest Surgery Clin N Am 2: 241-255

Okuhata Y et al. (1994) Inhalation MR lymphography: A new method for selective enhancement of the lung hilar and mediastinal lymph nodes. Magn Reson Imaging 12: 11351138

Padhani AR, Hale HL (1998) Mediastinal venous anomalies: Potential pitfalls in cancer diagnosis. Br J Radiol 71: 792798

Parker JR et al. (1997) Cellular hemangioma of the posterior mediastinum: Unusual presentation of a rare vascular neoplasm. J Okla State Med Assoc 90: 7-9

Rodriguez E et al. (1995) Massive mediastinal and cardiac fatty infiltration in a young patient. J Thorac Imaging 10 $225-226$

Roger T (1998) Mediastinal extramedullary hematopoiesis. J Belge Radiol 81: 248
Saleeb SF, Teplick SK (1999) General case of the day. Ectopic retrosternal parathyroid adenoma. Radiographics 19: 823825

Schaefer-Prokop C, Galanski M (1998) Mediastinum, Pleura und Thoraxwand. In: Galanski M, Prokop M (Hrsg) Ganzkörper-Computertomographie. Thieme, Stuttgart New York, S 177-200

Schüder G et al. (1995) Endosonographie von Speiseröhre und Mediastinum. Bildgebung 62, Suppl 2: 29-34

Schulman H et al. (1998) Ganglioneuroma: An ,incidentaloma“ of childhood. Eur Radiol 8: 582-584

Siegel MJ, Luker GD (1996) Pediatric chest MR imaging. Noncardiac clinical uses. Magn Reson Imaging Clin N Am 4: 599-613

Sippel JM et al. (1997) Extralobar pulmonary sequestration presenting as a mediastinal malignancy. Ann Thorac Surg 63: $1169-1171$

Slanetz PJ et al. (1998) Mediastinal lymphadenopathy and hazy mediastinal fat: New CT findings of congestive heart failure. Am J Roentgenol 171: 1307-1309

Soler R et al. (1996) MRI of mediastinal parathyroid cystic adenoma causing hyperparathyroidism. JACAT 20: 166168

Spiessl B et al. (1998) UICC. TNM atlas. Springer, Berlin Heidelberg New York Tokyo

Stansfield AG et al. (1988) Kiel classification. Lancet 1: 292-293

Storto ML et al. (1994) Spiral CT of the mediastinum: Optimization of contrast medium use. Eur J Radiol 18, Suppl I: S83-87

Strolle DC et al. (1997) Primary mediastinal tumors. Part II. Tumors of the middle and posterior mediastinum. Chest 112: 1344-1357

Taki S et al. (1996) Posterior mediastinal chordoma. Am J Roentgenol 166: 26-27

Topcu S et al. (1997) Mediastinal cystic lymphangioma: Report of two cases. Thorac Cardiovasc Surg 45: 209-210

Wegener OH (1992) Ganzkörpercomputertomographie. 2.Aufl., Blackwell, Berlin

Wilson D et al. (1994) Role of computed tomography scan in evaluating the widened mediastinum. Am Surg 60: 421-423 